\title{
Ultrasonic Evaluation of Beryllium-Copper Diffusion Bonds
}

Federal Manufacturing \& Technologies

E. E. Jamieson

KCP-613-6332

Published June 2000

Final Report

Approved for public release; distribution is unlimited.

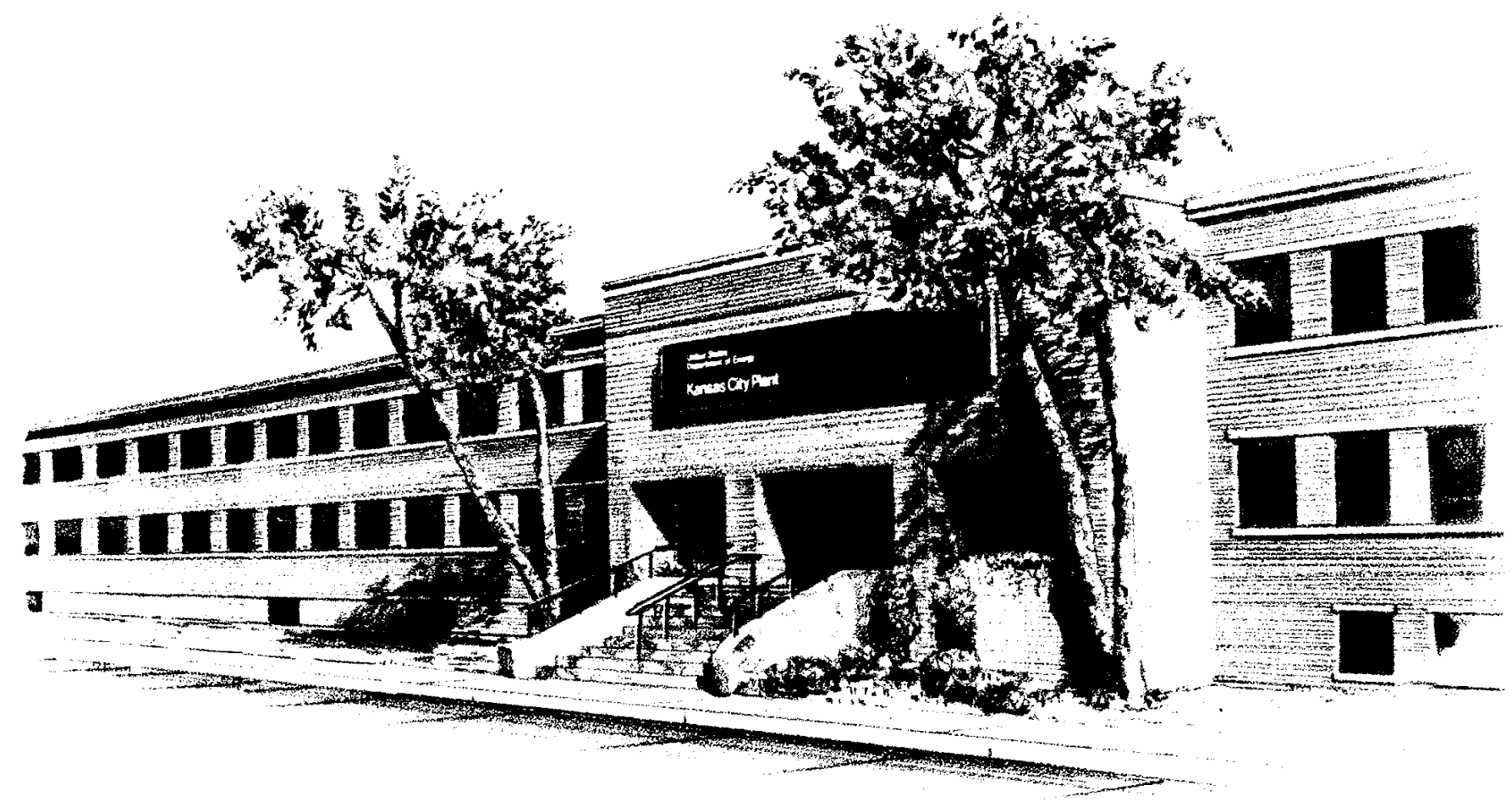




\section{DISCLAIMER}

This report was prepared as an account of work sponsored by an agency of the United States Government. Neither the United States Government nor any agency thereof, nor any of their employees, makes any warranty, express or implied, or assumes any legal liability or responsibility for the accuracy, completeness, or usefulness of any information, apparatus, product, or process disclosed, or represents that its use would not infringe privately owned rights. Reference herein to any specific commercial product, process, or service by trade names, trademark, manufacturer, or otherwise, does not necessarily constitute or imply its endorsement, recommendation, or favoring by the United States Government or any agency thereof. The views and opinions of authors expressed herein do not necessarily state or reflect those of the United States Government or any agency thereof.

All data prepared, analyzed and presented has been developed in a specific context of work and was prepared for internal evaluation and use pursuant to that work authorized under the referenced contract. Reference herein to any specific commercial product, process or service by trade name, trademark, manufacturer, or otherwise, does not necessarily constitute or imply its endorsement, recommendation, or favoring by the United States Government, any agency thereof or Honeywell International Inc.

Printed in the United States of America.

This report has been reproduced from the best available copy.

Available to DOE and DOE contractors from the Office of Scientific and Technical Information, P. O. Box 62, Oak Ridge, Tennessee 37831; prices available from (865) 576-8401, FTS 626-8401.

Available to the public from the National Technical Information Service, U. S. Department of Commerce, 5285 Port Royal Rd., Springfield, Virginia 22161, (703) 487-4650.

A prime contractor with the United States Department of Energy under Contract Number DE-ACO4-76-DP00613.

\author{
Honeywell \\ Federal Manufacturing \\ \& Technologies \\ P. O. Box 419159 \\ Kansas City, Missouri \\ 64141-6159
}




\section{Honeywell}

KCP-613-6332

Distribution Category UC-706

Approved for public release; distribution is unlimited.

\section{ULTRASONIC EVALUATION OF BERYLLIUM-COPPER DIFFUSION BONDS}

E. E. Jamieson

Published June 2000

Final Report

E. E. Jamieson, Project Leader 


\section{Contents}

Section

Abstract

Summary

Discussion

Scope and Purpose

Prior Work

Activity

Ultrasonic Techniques for Bond Evaluation

Experimental Procedures

Results

Accomplishments

Future Work

References

Acknowledgments

Appendices
A. Sample Design
B. Diffusion Bond Equipment
C. Diffusion Bond Parameters
D. Computer Code
E. Reflection Coefficient and TOF Histogram Plots
F. Metallographic Images of the Bond Interface 


\section{Illustrations}

Figure

1 Oscilloscope Display of Acoustic Signal From Bonded Sample

2 Bond Quality Versus Reflection Coefficient Graph

3 Squared and Averaged RF Waveform From Half Sample Frequency Domain Reflection Coefficients

4 Averaged Reflection Coefficient for Sample \#10

5 Averaged Frequency Dependent Reflection Coefficients for Perfect Bonds and Perfect Unbonds

6 Method of Recording Peak Voltage and Arrival Time

7 Time-of-Flight Histogram of Half Sample

8 Time-of-Flight Histogram of Solid Sample

9 Block Diagram of Diffusion Bond Process

10 Bonded and Half Samples of Beryllium-Copper

11 Block Diagram of Ultrasonic Test System

12 Scan Path Across Half Sample

13 C-scan Images of Half Sample and Ideally Perfect Bond

14 Oscilloscope Display of Electronic Gate Placement

15 Graphical Output From IDL Computer Code

16 Integrated Backscatter Versus Tensile Strength

17 Time-of-Flight Variance Versus Tensile Strength

18 C-scan Image of Sample \#8

19 C-scan Images of Samples \#3, \#12, \#19, and \#22

20 Reflection Coefficient Slope Versus Tensile Strength

21 Reflection Coefficient Y-Intercept Versus Tensile Strength

A1 Beryllium-Copper Diffusion Bond Sample Design

B1 Diffusion Bond System and Fixturing

B2 Diffusion Bond Fixture

B3 Pre-etch Surface Profile Measurement on Sample 12B

B4 Post-etch Surface Profile Measurement on Sample 12B

F1 Sample 6-1A-C Cross Section

F2 Sample 6-1A-D Cross Section

F3 Sample 6-3A-C Cross Section

F4 Sample 6-3A-D Cross Section

F5 Sample 6-4A-C Cross Section

F6 Sample 6-4A-D Cross Section

F7 Sample 9-1A-C Cross Section 
F8 Sample 9-1A-D Cross Section

F9 Sample 9-2A-C Cross Section

F10 Sample 9-3A-C Cross Section

F11 Sample 9-3A-D Cross Section

F12 Sample 9-4A-C Cross Section

F13 Sample 9-4A-D Cross Section

F14 Sample 2 Face A - 1000X

F15 Sample 2 Face A - 5000X

F16 Sample 19 Face A - 1000X

F17 Sample 19 Face A - 5000X

F18 Sample 8 Face A - 1000X

F19 Sample 8 Face A - 5000X

\section{Tables}

Number

1 Scan and Waveform Digitizing Parameters

2 Waveform Array Processing Steps for IDL Code

3 Tensile Test Data and Calculated Acoustic Parameters

4 Regression Analysis $R^{2}$ Values

B1 Equipment List for Beryllium-Copper Sample Preparation and Diffusion Bond Procedures

C1 Diffusion Bond Parameters 


\begin{abstract}
A study was performed to compare the effectiveness of several advanced ultrasonic techniques when used to determine the strength of diffusion bonded beryllium-copper, which heretofore have each been applied to only a few material systems. The use of integrated backscatter calculations, frequency domain reflection coefficients, and time-of-flight variance was compared in their ability to characterize the bond strength in a series of beryllium-copper diffusion bond samples having a wide variation in bond quality. Correlation of integrated backscatter calculations and time-of-flight variance with bond strength was good. Some correlation of the slope of the frequency based reflection coefficient was shown for medium and high strength bonds, while its Y-intercept showed moderate correlation for all bond strengths.
\end{abstract}

\title{
Summary
}

Conventional peak amplitude ultrasonic techniques have proven to be unreliable when used to evaluate solid state bonds. Of particular concern are bonds of intermediate strength that would pass a typical ultrasonic examination. These are commonly called "kissing bonds." Several advanced ultrasonic techniques were compared to determine the best technique for predicting the bond strength of diffusion bonded beryllium-copper. After an extensive literature search, several advanced ultrasonic techniques were chosen for inclusion in this study. Integrated backscatter calculations, frequency domain reflection coefficients, and time-of-flight variance techniques, which showed varying degrees of success on other material interface systems, were compared in their ability to characterize the bond strength of twenty-two beryllium-copper diffusion bond samples with varying bond qualities. Modifications to previously reported time-of flight histogram and reflection coefficient techniques were also suggested and implemented as a part of this study.

Correlation of integrated backscatter calculations and time-of-flight variance with bond strength was good for low, intermediate, and high strength bonds. Correlation of the slope of the frequency based reflection coefficient was poor when considering all bond strengths; however, some correlation was found for medium and high strength bonds. The Y-intercept of the frequency based reflection coefficient curve showed moderate correlation for all bond strengths. 


\section{Discussion}

\section{Scope and Purpose}

Diffusion bonding is a joining process that is becoming increasingly popular as a metallurgical joining method, and offers many advantages over other processes such as fusion welding and brazing. It allows similar and dissimilar metals to be joined at temperatures that are not only far below their melting temperature, but in many cases below their heat treating and annealing ranges. It can also avoid the drastic changes in microstructure typically seen in and adjacent to fusion welds, and the numerous undesirable conditions that can occur during the solidification process. The diffusion bonding process involves applying pressure at an elevated temperature to two samples which have their mating surfaces prepared as flat and smooth as possible. Initially, diffusion begins where contact is made between the two surfaces at the highest points. As the pressure and heat are increased, plastic deformation causes the interface to transform into one of isolated voids, thus increasing the contact area and the rate of diffusion. The voids are then reduced in size until they are completely closed, at which time the rate of atomic diffusion is a maximum. This process is accomplished principally by interstitial or vacancy diffusion, depending upon the materials at the interface $(1,2)$.

The quality of a diffusion bond, as in other bonds, is of critical importance in ensuring the proper performance of the bond for its intended application. Nondestructive test methods, particularly ultrasonic techniques, have been a prime area for research in determining the quality of diffusion bonds. Ultrasonic techniques, in comparison with other nondestructive methods, offer the most sensitivity for evaluating metallurgical bonds due to the fact that acoustic properties of materials and interfaces are closely related to their elastic properties, as shown in the following equation for longitudinal acoustic velocity:

$$
C_{L}=\sqrt{\frac{E}{\rho} \frac{(1-v)}{(1+v)(1-2 v)}}
$$

where $\quad E=$ modulus of elasticity

$$
\rho=\text { density, }
$$

and

$$
v=\text { Poisson's Ratio. }
$$

This investigation compares several ultrasonic techniques in their ability to characterize the strength of beryllium-copper/beryllium-copper diffusion bonds. A series of diffusion bonds was made which attempted to produce wide variations in the bond line condition. Of particular interest are bonds in which voids have been eliminated at the bond line but little or no diffusion occurs, producing a low strength bond which would be accepted using conventional ultrasonic techniques. Bonds yielding these characteristics are commonly referred to as "kissing bonds." 


\section{Prior Work}

No prior work on this problem has occurred at Federal Manufacturing \& Technologies (FM\&T). However, the use of ultrasonic techniques as a method to evaluate solid state bonds has been a common area for research at leading research laboratories over the past two decades. The primary motivation in these studies is to develop advanced ultrasonic methods that overcome the inability of conventional techniques in characterizing bonds, particularly those having intermediate strength. This is primarily due to the bond condition where strong reflectors (voids) have disappeared from the bond line, but insufficient diffusion has occurred to produce high strength, thus producing the 'kissing bond.' In bonds of similar materials, the reflected energy from the bond is reduced to approximately the same magnitude as the energy backscattered from the grains of the material at and adjacent to the bond interface. Thus, the lack of strong reflectors precludes the use of conventional peak amplitude methods.

A different problem arises in the case of dissimilar bonds, wherein a reflection from the bondline is expected based upon the relationship in acoustic impedance between the two bonded materials. In this case, intimate contact at an interface without diffusion has been observed to cause a reflection having the same amplitude as that predicted from a good bond (4). Fortunately, advancements in instrument electronics, transducer design, and the use of high speed digital processing have promoted many new techniques to be developed which offer improved sensitivity in the evaluation of these conditions. Numerous analytical and empirical approaches have been reported to date with moderate success in characterizing bond strength. Thomas and Spingarn, for instance, used pattern recognition programs to identify five time and frequency features in waveforms which correlated to bond strength, resulting in the development of a weighted classification algorithm $(5,6)$. Buck et. al. correlated the electromechanical reciprocity theorem and a spring-mass-damping model to bond strength in copper-copper diffusion bonds (7). A method utilizing transfer functions and eigenvectors was introduced by Kato and Abe which showed moderate success in predicting strength of steel/titanium diffusion bonds (8). Two promising techniques were reported in which determination of a single frequency reflection coefficient and the calculated total energy at the bond line were correlated with bond strength. Ojard et. al. reported good correlation on similar bonds of copper/copper by using both single frequency reflection coefficients and normalized energy calculations (9). Ojard et. al. also reported success in utilizing the normalized energy calculations on dissimilar bonds of copper/nickel; however, the single frequency reflection coefficients were unsuccessful in correlating with bond strength $(4,9)$. A novel approach was reported by Nieters et. al. where time-of-flight (TOF) histograms were very successfully used to identify incomplete diffusion bonds in titanium samples (10).

Following review of the aforementioned literature, three of the concepts - normalized energy, reflection coefficient, and TOF histograms - were chosen for this experiment because of their effectiveness in characterizing diffusion bonds of similar materials. Enhancements to reported reflection coefficient and TOF techniques are also introduced in this work. 


\section{Activity}

\section{Ultrasonic Techniques for Bond Evaluation}

\section{Introduction to Ultrasonic Inspection Techniques}

As an acoustic wave interacts with any interface between two materials, the conservation of energy dictates that a portion energy will be transmitted through the interface, while the remaining energy will be reflected, with incidental losses occurring through absorbed energy being converted to heat. Perhaps the most common ultrasonic technique used for evaluation of bonds is the measurement of the reflected energy from the interface. This is primarily accomplished through monitoring of the peak amplitude of the signal reflected from the interface. Though it will not be used as a primary technique in this experiment, a description of its fundamentals will serve as a basis for reference when describing more advanced techniques. All data in this experiment was acquired using the immersion pulse-echo technique, whereby a piezoelectric transducer with a focusing lens transmits an acoustic wave through the coupling medium (water), and then receives acoustic waves reflected from the sample of interest. The voltage output of the transducer is displayed on an oscilloscope, where the time base of the oscilloscope represents the distance traveled by the acoustic waves. A typical oscilloscope display from a diffusion-bonded sample is shown in Figure 1. During data collection, the peak voltage of the reflected signal from the bond interface is digitized, and represents the amount of energy reflected from an interface. The amplitude ratio of the reflected and incident waves is called the reflection coefficient. For broadband time domain RF signals at an ideal stress-free boundary, it is described as

$$
R=\frac{\sin 2 \phi_{I} \sin 2 \phi_{T}-\kappa \cos ^{2} 2 \phi_{T}}{\sin 2 \phi_{I} \sin 2 \phi_{T}+\kappa \cos ^{2} 2 \phi_{T}}
$$

where $\mathrm{R}=$ the reflection coefficient for the reflected longitudinal wave;

$\phi_{I}=$ angle of the incident longitudinal wave

$\phi_{T}=$ angle of the refracted transverse wave

$\kappa=\left(\frac{c_{L}}{c_{T}}\right)$, the ratio of longitudinal and transverse wave speeds

or $\quad \kappa=\frac{2(1-v)}{1-2 v}$ 


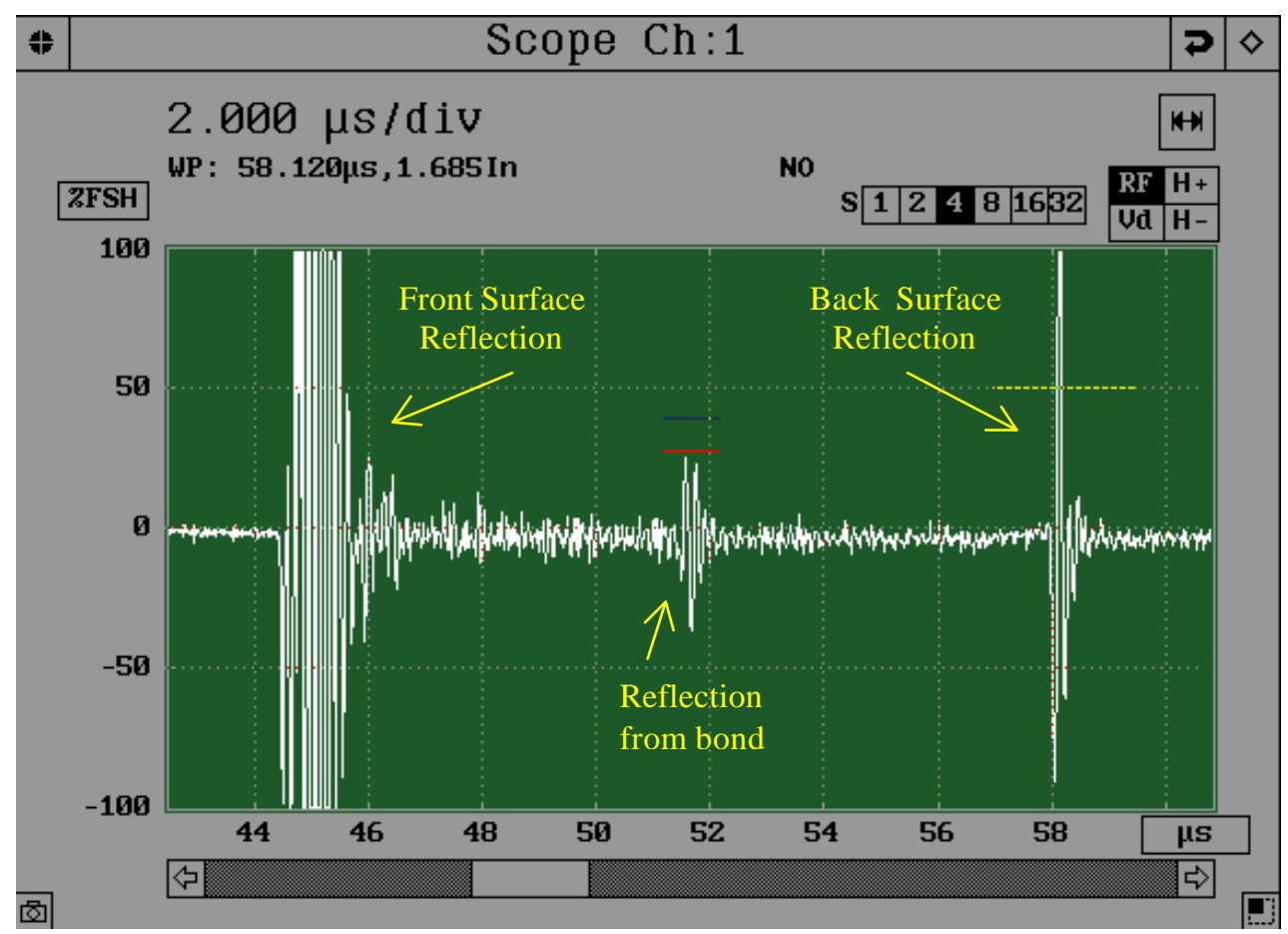

Figure 1. Oscilloscope Display of Acoustic Signal From Bonded Sample

If the incident angle is $90^{\circ}$, the equation for $\mathrm{R}$ can be more simply expressed using $\mathrm{Z}$, acoustic impedance, as follows:

$$
R=\frac{Z_{2}-Z_{1}}{Z_{2}+Z_{1}}
$$

where $\mathrm{Z}=C_{L} * \rho$, acoustic impedance

and $\rho=$ material density

With respect to diffusion bond quality, an ideally perfect unbond (beryllium-copper/air) would yield a reflection coefficient of 0.99978 , whereas an ideally perfect bond would yield a reflection coefficient of 0.0, leaving only backscattered grain noise as the dominant signal. For bonds of poor to intermediate quality the reflection coefficient is generally inversely proportional to the bond quality, meaning that the peak amplitude decreases with increasing bond quality. This relationship has been observed to be invalid, however, when the kissing bond condition is encountered. The above statements are shown graphically in Figure 2. The calculations just described determine the reflection coefficient for a time domain signal, having broad band qualities with a range of frequencies existing within the envelope of the signal. Reflection 


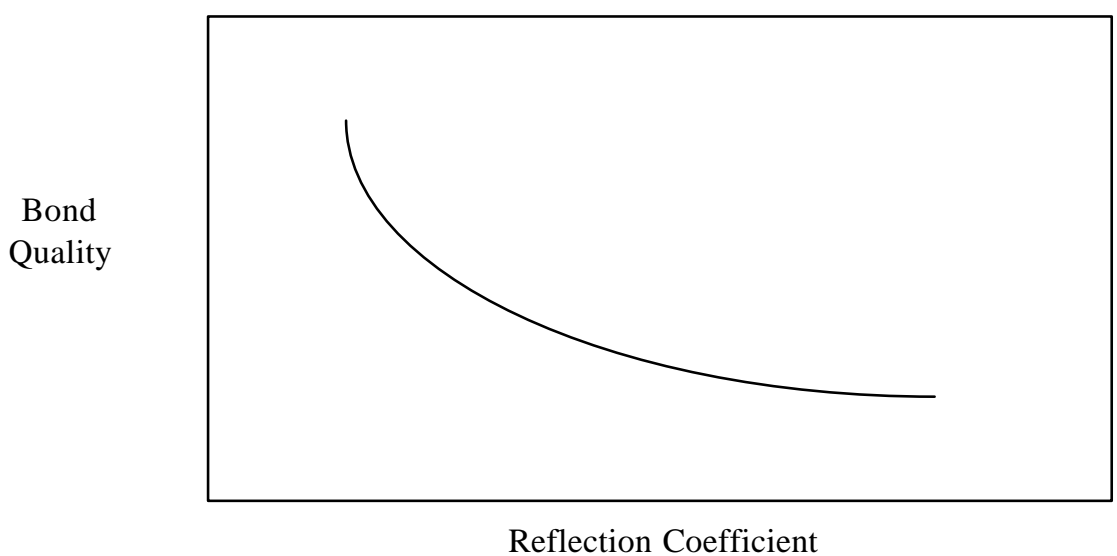

Figure 2. Bond Quality Versus Reflection Coefficient Graph

coefficients may also be calculated in the frequency domain at specific frequencies by processing a signal from the bond line with a reference signal. This is accomplished digitally by performing a Fast Fourier Transform (FFT) on the bond line signal and a reference signal, and then taking the ratio of the magnitudes of the two spectra. The result is a reflection coefficient at each discrete frequency produced within the transform.

\section{Integrated Backscatter}

The integrated backscatter (IBS) calculation is one method of measuring of the total energy reflected from the bond line. Though energy is typically computed via the power spectrum in the frequency domain, Parseval's Theorem states that the energy in the time domain is equal to the energy in the frequency domain, as shown in the following expression:

$$
\int_{-\infty}^{+\infty}[g(t)]^{2} d t=\int_{-\infty}^{+\infty}[G(f)]^{2} d f
$$

This allows a convenient method of calculation by summing the squares of the peak voltage of the reflected wave in the time domain. Ojard found that the sensitivity of these types of measurements was enhanced over the single frequency reflection coefficient measurement (9). In this experiment, a single averaged IBS value is calculated for correlation with bond strength by a) squaring the voltages within each waveform from a sample, b) averaging all squared waveforms, and c) summing the squared voltage values in the averaged waveform. The result of averaging the squared waveforms from a half sample is shown in Figure 3. 
Squared and Averaged RF Waveform from Half Sample

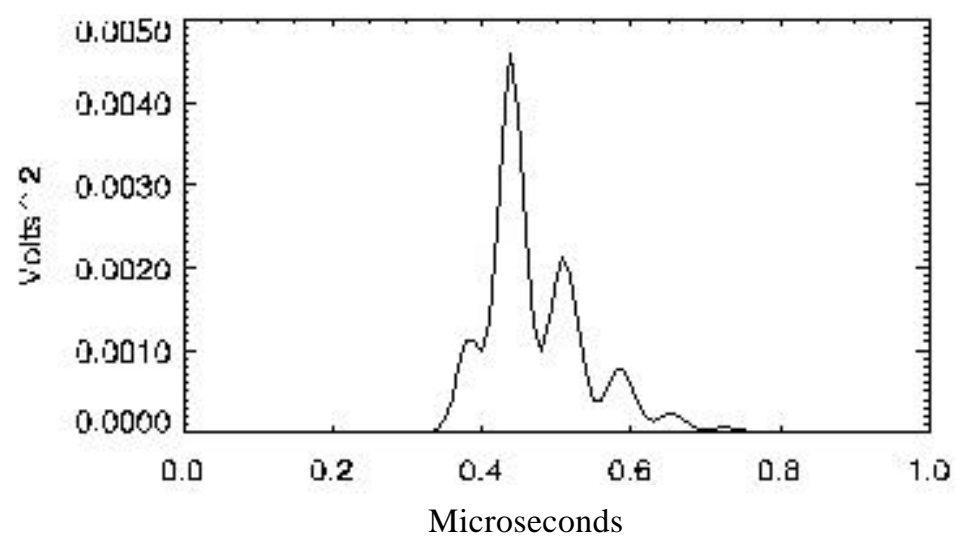

Figure 3. Squared and Averaged RF Waveform From Half Sample

\section{Frequency Domain Reflection Coefficients}

It was found by D. D. Palmer et. al. that the reflection coefficient measured at a specific frequency or frequencies showed correlation with bond strength in diffusion bonds of pure copper (11). In this approach, the time domain signals from a test sample are transformed into the frequency domain using the Fast Fourier Transform (FFT). The resultant complex elements are then used to compute the magnitude spectra by taking their absolute value. Similar treatment of the waveforms taken from an ideally imperfect bond (i.e., a half sample) are then performed. The ratio of the two magnitude spectra results in a frequency dependent reflection coefficient array. Mathematical treatment of acoustic waves reflected from an interface usually involves an understanding of additional parameters such as the transducer characteristics, beam divergence, and attenuation, as shown in the following equation for a reference sample:

$$
F_{R}(f)=E(f) e^{-\alpha_{w}(f) d w} c(f, d e) T_{w s} e^{-\alpha_{s}(f) d s} R_{s w} T_{s w}
$$

$$
\begin{aligned}
& \text { where } \quad \begin{array}{l}
F_{R}=\text { Fourier } \text { Transform of the measured signal } \\
E=\text { transducer } \text { and electronic effects } \\
\alpha=\text { attenuation coefficient } \\
c=\text { combined diffraction effect for water and solid } \\
T=\text { transmission coefficient } \\
R=\text { reflection coefficient } \\
w s=\text { water/solid interface } \\
\text { and } \quad s w=\text { solid/water interface }
\end{array}
\end{aligned}
$$


However, by taking the ratio of the spectra from a bond sample and a reference, all of the aforementioned terms cancel and greatly simplify the calculation as shown below.

$$
\left|\frac{F_{B}(f)}{F_{R}(f)}\right|=\left|\frac{E(f) e^{-\alpha_{w}(f) d w} c(f, d e) T_{w s} e^{-\alpha_{s}(f) d s} R_{B} T_{s w}}{E(f) e^{-\alpha_{w}(f) d w} c(f, d e) T_{w s} e^{-\alpha_{s}(f) d s} R_{s w} T_{s w}}\right|=\left|\frac{R_{B}}{R_{s w}}\right|
$$

It should be noted that the transformed signal from the reference, which is a distinct interface between two materials having significantly different acoustic properties, is a surface integral and is frequency independent. Conversely, the transformed signal from a diffusion bonded sample represents an interface between two identical materials with the pore volume eliminated, producing only backscattered signals due to grains at and near the bond line, and therefore constitutes a volume integral which is frequency dependent. Consequently, the ratio of a transformed sample and reference signal in this application does not represent a true reflection coefficient in the pure sense, but is an approximation wherein the purpose is to highlight subtleties in frequency dependence of the sample.

A typical reflection coefficient spectrum is shown in Figure 4. Palmer, et. al. correlated bond strength with only a single frequency reflection coefficient. It is clear, however, from both theoretical models and experimental data $(2,7)$ that reflection coefficients vary as a function of frequency with respect to bond quality. The unique approach taken in this experiment attempts to utilize all frequencies which contribute to the reflected energy by finding correlation between the slope of the function representing the averaged reflection coefficients between 5 and $15 \mathrm{MHz}$ (the dominant frequencies in the spectrum) and the ultimate strength of each specimen. In the worst case of an ideally imperfect bond, a large reflection is expected from the bond line which is identical in frequency content and magnitude to the reference signal (also obtained from an ideally imperfect bond). The ratio of the magnitude spectra from the two bonds results is a curve having reflection coefficients of 1 at all frequencies, yielding a slope of zero as shown in Figure 5. For an ideally perfect bond for which there is no distinguishable signal above the backscattered noise, the ratio of its corresponding magnitude spectra with the reference spectra will yield a curve having a positive slope and a correspondingly lower Y-intercept, also shown in Figure 5. The dominant frequencies for this material and transducer combination were determined by observing the average $-8 \mathrm{~dB}$ bandwidth centered around the peak frequency. This resulted in utilizing the frequency band from $5 \mathrm{MHz}$ to $15 \mathrm{MHz}$ for reflection coefficient calculations. 
Averaged Reflection Coefficient for Sample \#10



Figure 4. Averaged Reflection Coefficient for Sample \#10



Figure 5. Averaged Frequency Dependent Reflection Coefficients for Perfect Bonds and Perfect Unbonds 


\section{Time-of-Flight Variance Technique}

The time-of-flight (TOF) histogram technique is a novel approach that has been reported by Nieters et. al., to successfully characterize diffusion bonded titanium (10). In this technique, the arrival time of the peak signal is recorded, and then a histogram of the data is constructed, as shown in Figure 6. For a poor bond which produces a strong reflection, the arrival time of the peak reflected signal will have little variation, and produce a histogram having all data points concentrated in a narrow region of time equivalent to the depth of the bond plane as shown in Figure 7. In the case of a perfect bond, the arrival time of the peak backscattered sound from the grains is random, and produces a histogram with a relatively normal distribution, as shown from a solid sample in Figure 8. At the point in the diffusion bonding process when there is complete closure of the pores, all strong reflectors at the bond line have disappeared, resulting in no distinguishable reflected signal above that produced by the random backscattering from individual grains, or "noise." If the diffusion process has not progressed to the point where new grains have completely formed at the bond line, a portion of the grains will exhibit a boundary coincident with the bond line. The consequence of these planar boundary arrays is a weaker bond than one with complete grain growth. The lack of strong reflectors precludes the use of the peak amplitude as an effective technique for this case. The TOF histogram technique takes advantage of the fact that the arrival time of the peak backscattered sound will be preferentially oriented around boundaries coincident with the original bond line, thus producing a histogram showing a distinct peak. Nieters et. al. reported a qualitative correlation between the TOF histogram results and evaluation of the bond interface and grain boundaries of cross-sectioned samples (10). This experiment extends that work by correlating the TOF data quantitatively with bond strength by computing its variance. For a poor bond, the variance would yield a correspondingly very low value, while for an ideally perfect bond a relatively normal TOF distribution would occur, yielding a variance with a correspondingly high value.



Figure 6. Method of Recording Peak Voltage and Arrival Time 
TOF Histogram of Half Sample

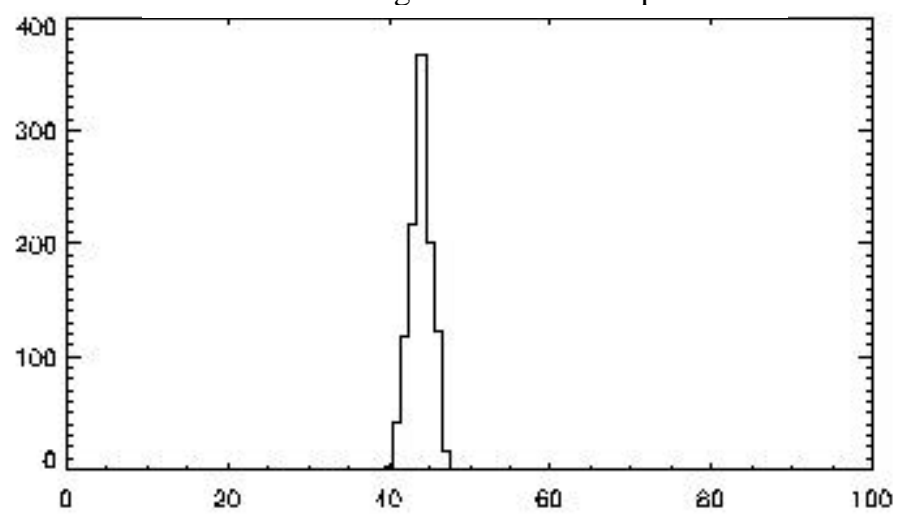

Figure 7. Time-of-Flight Histogram of Half Sample

TOF Histogram of Solid Sample

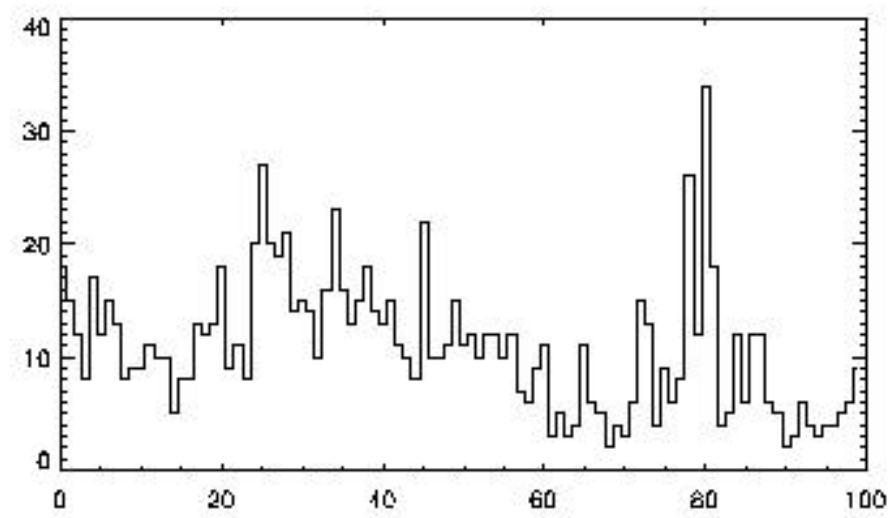

Figure 8. Time-of-Flight Histogram of Solid Sample

\section{Experimental Procedures}

\section{Diffusion Bonding Method}

Development of a method for diffusion bonding of beryllium copper was reported by D. Eckman (12). In his work, complete grain growth across the bond was not achieved; however, the maximum bond strength did reach $85 \%$ of the ultimate strength of the material. The procedure involved a simple fixture designed to apply a designated compressive force to two half samples under a high vacuum and heat. The same basic experimental procedure used by Eckman was employed for this project, with minor modifications implemented to attempt to improve process control. The main improvement involved an improved fixture design which ensured a normal force was applied to the bond specimens. A block diagram of the process is shown in Figure 9. 


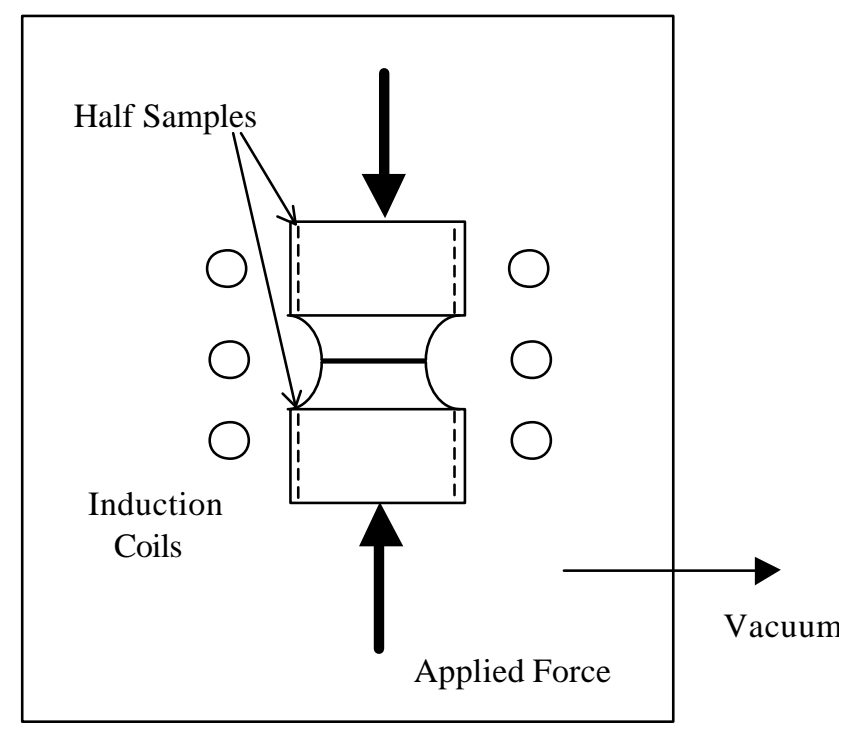

Figure 9. Block Diagram of Diffusion Bond Process

\section{Material, Sample Design and Preparation}

The material chosen for this experiment was $\mathrm{BeCu}$ alloy no. $172,(2 \% \mathrm{Be}$, balance $\mathrm{Cu})$ which is a precipitation hardenable alloy, with the gamma phase, $\mathrm{BeCu}$, being the stable precipitate. It was purchased in the solution annealed condition. Samples of beryllium-copper were designed and fabricated to be conceptually similar to an ASTM tensile specimen for ease of mechanical testing. The bond surface diameter is 0.5 inch, and 3/4 -16 U.N.F. threads were used to help minimize the total length of the bonded specimen. Holes having a diameter of 0.021 inch were added near each end to facilitate the use of thermocouples. A photograph of a bonded sample and half sample is shown in Figure 10. The samples were polished flat, resulting in surface roughnesses (peak-to-peak) varying from $500 \AA$ to $10,000 \AA$. This was accomplished by mounting four to six half samples on a glass plate, and lapping the surfaces using various polishing compounds on surface plates. The lapping process consisted of four successive steps, beginning with 15-micron polishing compound, then proceeding to 9-micron, 6-micron, and finishing with 3-micron. Just prior to bonding, each sample was chemically etched with a $6 \%$ sulfuric acid, 3\% nitric acid, and 91\% de-ionized water solution to remove oxides and other contaminants from the surface as well as reveal some new layers of copper. However, upon observing a wide variation in ultrasonic data on several samples bonded early in the experiment at the same time, temperature, and pressure, it became clear that an additional parameter or parameters were strongly contributing to the variations in bond quality. The most likely of all processing parameters and the most difficult to control was the cleanliness of the bond surfaces. This seemed evident after observing different reaction rates from sample to sample when performing the chemical etch process. Because the chemical etch concentration and temperature were controlled, it seems probable that contamination of the bond surface in the form of 


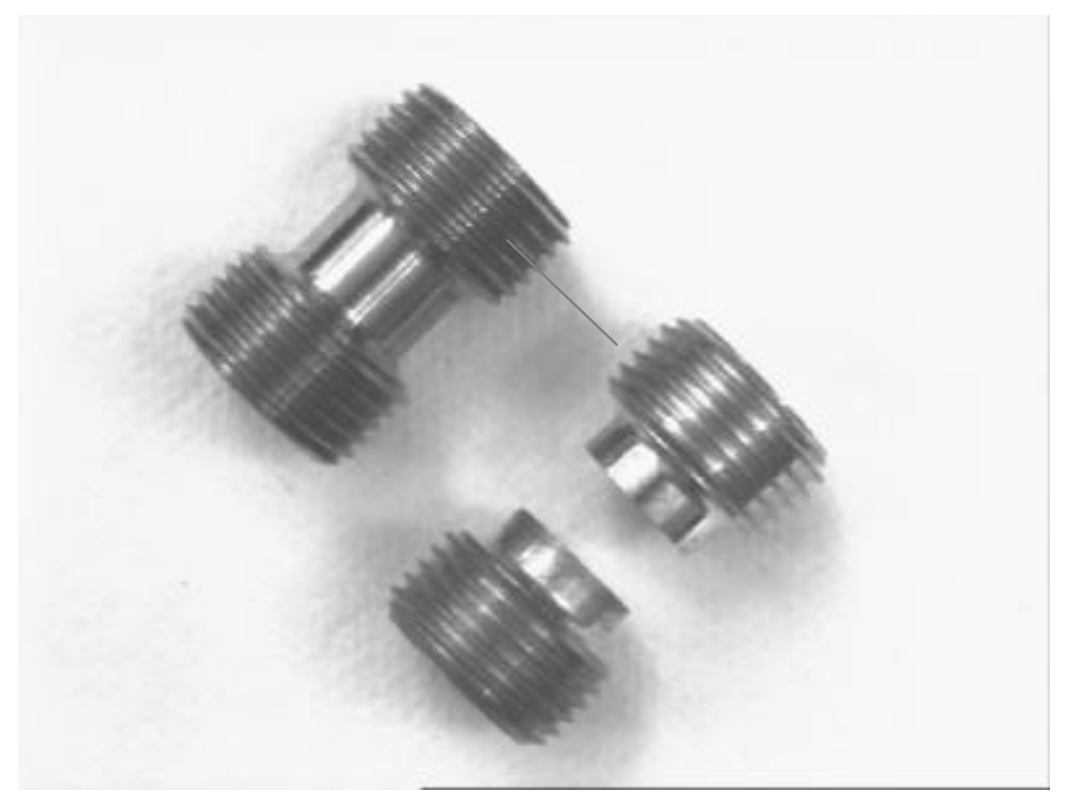

Figure 10. Bonded and Half Samples of Beryllium-Copper

hydrocarbons or varying layers of oxides was responsible for the differing chemical reaction rates. If unaffected by the etching process, the contaminants could impede the diffusion reaction during the bonding process. Additional cleaning steps then were added during sample preparation. However, upon diffusion bonding several additional samples, all at time, temperature, and pressure settings sufficient to produce a high strength bond, wide variations, were again observed in the ultrasonic data, suggesting that the added cleaning steps were not effective. Realizing that producing a wide variation in bond quality was one of the key goals in the experiment, and that was in fact occurring though not in a well-controlled fashion, the remainder of the samples were produced using parameters to attain the highest quality bond possible, with results similar to the previous bond experiments.

\section{Diffusion Bond Experiments}

All diffusion bond experiments were conducted using a vacuum system, induction heating system, and fixturing which are described in detail in Appendix B. Two polished and cleaned samples were loaded into the diffusion bond fixture, and two type $\mathrm{K}$ thermocouples were inserted into prepared holes. The system was then evacuated to a pressure of between 1.0 E- 6 torr and 0.1 E-7 torr, and heated to a temperature of approximately $800^{\circ} \mathrm{C}$. For all but three of the bond samples, a force of 100 pounds was applied to the force rod, exerting a pressure of approximately 511 pounds/in ${ }^{2}$ (psi) nominal at the bond line. The force was modified on samples \#5, \#7, and \#16 to observe the effect on the bond quality. As explained in the previous section, time was initially the primary parameter to be adjusted to produce variations in bond quality. However lack of control over cleanliness of the bond surface dictated the time, temperature, and force parameters to be at settings which are known to produce the best possible bond. Bonding parameters for all samples are listed in Appendix C. 


\section{Ultrasonic Measurement Procedures and Experimental Data}

\section{Ultrasonic Equipment}

Ultrasonic RF waveform data was acquired using a six-axis digital ultrasonic c-scan system and software. A block diagram of the system is shown in Figure 11. The system is comprised of a personal computer having an internal analog/digital converter board and six-axis motion control board, a computer-controlled pulser/receiver, and six-axis scanning tank. Software controls all aspects of data acquisition, motion control, ultrasonic pulser/receiver settings, data archival, and image display. The transducer used for this experiment had a center operating frequency of $15 \mathrm{MHz}$ (nominal), piezoelectric element diameter of 0.5 inch, and a spherical focusing lens achieving a nominal focal length of 3 inches in water.



Figure 11. Block Diagram of Ultrasonic Test System

\section{General Measurement Procedure}

Data was acquired from both sides of each sample with the sample fixed and the transducer raster scanned across the sample, as illustrated in Figure 12. The transducer was positioned at a water path of 44.5 microseconds with normal incidence to facilitate focusing at the bond plane. This was determined experimentally on a half sample. Though a square plot does not cover the entire cross-sectional area of the bond, it was chosen over a polar plot to maintain a constant spatial resolution and to avoid distortion of the beam near the edges of the specimen. Plotting the peak amplitude of the bondline signal using the scan path shown in Figure 12 generates a 


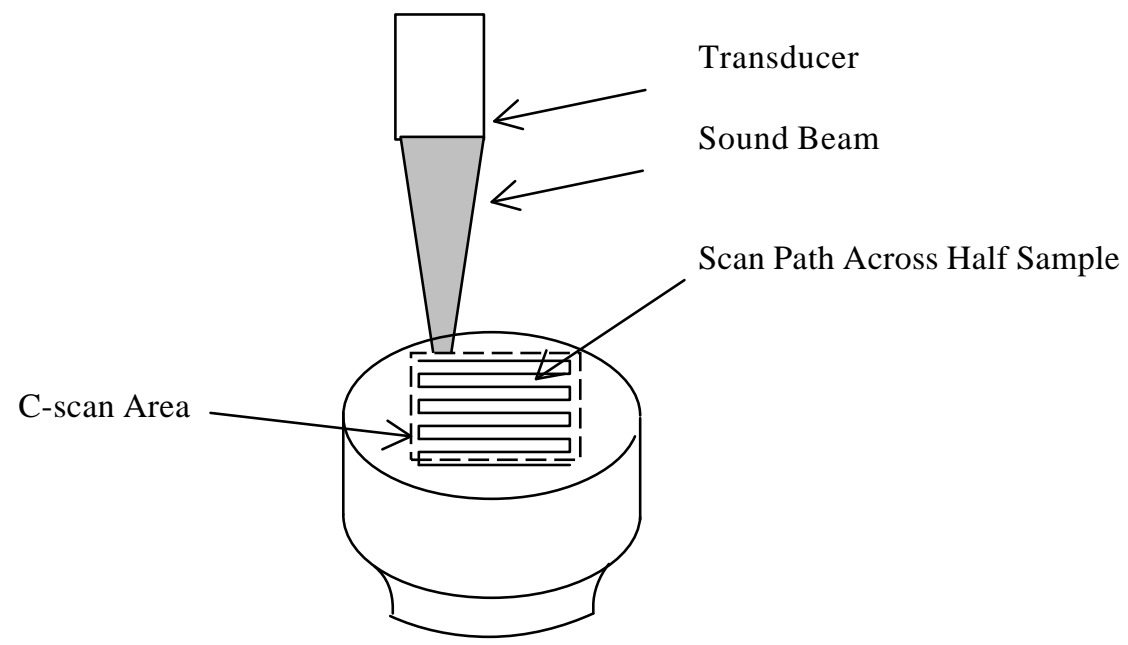

Figure 12. Scan Path Across Half Sample

plan view image of the sample known as a $c$-scan. C-scan images from a half sample and ideally perfect bond are shown in Figure 13. Preliminary peak amplitude c-scans were performed on each sample to ensure appropriate instrument settings and that the scan was centered on the sample. Receiver gain and A/D board sensitivity were adjusted for each sample to maintain the bondline signal at an average level of at least $35 \%$ full screen yet not saturated.

In the final scan, full RF waveforms were acquired at each data point within the scan. Frame averaging was used to minimize electronic noise. Only RF data from the bondline area was acquired for analysis; therefore an electronic gate was centered at the mid-point of the front and back surface reflections. The gate was 1 microsecond in length, allowing 100-point RF waveforms to be acquired at a digitizing rate of 100 Megahertz (MHz). An example of the gate placement is shown in Figure 14. Table 1 summarizes the measurement conditions. The waveforms are digitized using an 8 bit A/D converter, with the raw data from each waveform being stored in the file as a string of 100 binary numbers ranging from 0 to 255 .

\section{Experimental Data and Analysis}

\section{I.D.L. Computer Code for Ultrasonic Data Analysis}

Ultrasonic analysis of digital RF data was performed using computer code written in the Interactive Data Language (IDL), manufactured by Research Systems, Inc., Boulder, CO. A subroutine was first developed to read the data file produced by the digital ultrasonic system, providing file size information and a pointer to the beginning of the raw data. The main IDL code was then developed to process the raw data, which in this experiment consisted of $1089 \mathrm{RF}$ waveforms in a $33 \times 33$ array. The program is listed in full in Appendix D. An outline of the processing steps is listed in Table 2 . The main program processes each waveform individually after reading all 1089 waveforms into a three-dimensional array, structured as follows: (rfwaveform, datapoint, row). The first processing step performed on a given waveform is 


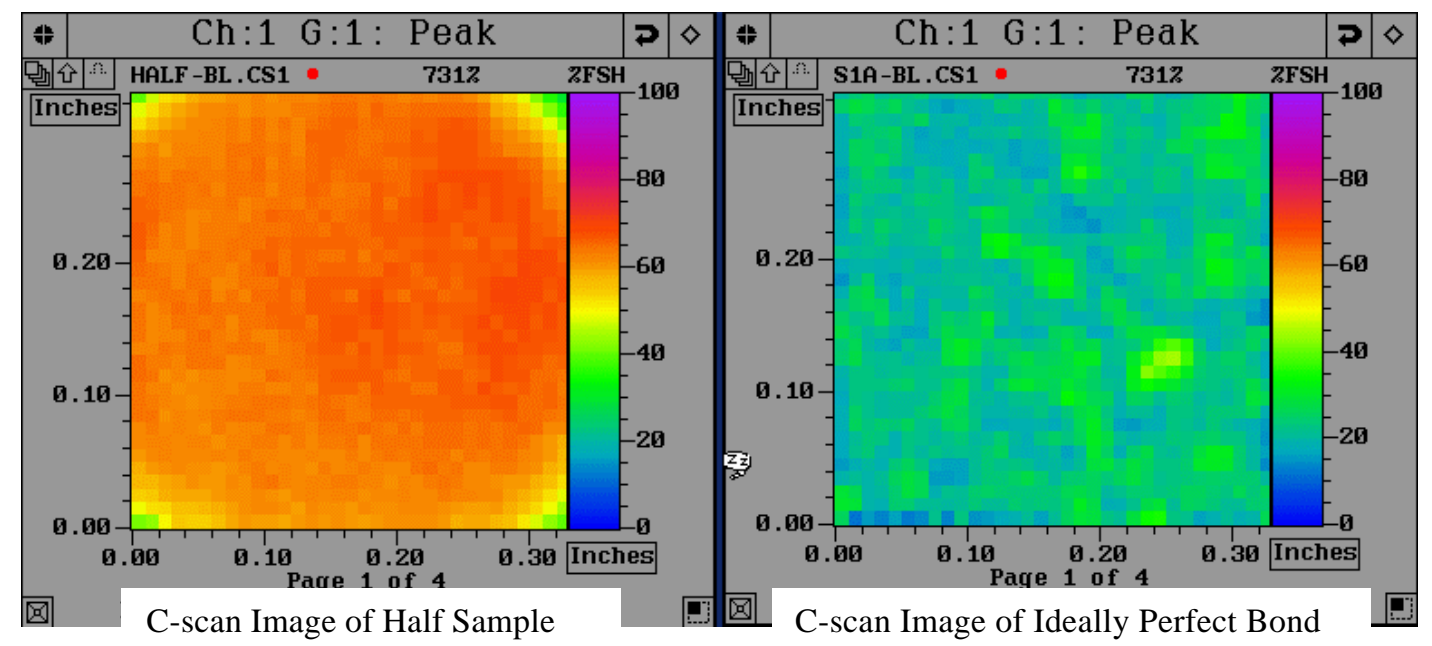

Figure 13. C-scan Images of Half Sample and Ideally Perfect Bond



Figure 14. Oscilloscope Display of Electronic Gate Placement 
Table 1. Scan and Waveform Digitizing Parameters

\begin{tabular}{|l|c|}
\hline Scan Size & 0.33 inches x 0.33 inches \\
\hline Scan Resolution (X \& Y direction) & 0.010 inches \\
\hline Total Number of Data Points & 1089 \\
\hline RF Waveform (One Data Point) & 100 points $(1 \mu \mathrm{sec} @ 100 \mathrm{MHz})$ \\
\hline
\end{tabular}

Table 2. Waveform Array Processing Steps for IDL Code

\begin{tabular}{|c|c|}
\hline Step & Process \\
\hline 1 & Read in sample waveform file \\
\hline 2 & Normalize first waveform \\
\hline 3 & Convert 8 bit waveform values to voltages \\
\hline 4 & $\begin{array}{c}\text { Compute IBS and TOF values, and average magnitude (from FFT) } \\
\text { of waveform }\end{array}$ \\
\hline 5 & Repeat Steps 2-4 for each remaining waveform \\
\hline 6 & Read in reference waveform file \\
\hline 7 & Normalize first reference waveform \\
\hline 8 & Convert 8 bit reference waveform values to voltages \\
\hline 9 & $\begin{array}{c}\text { Compute IBS and TOF values, and average magnitude (from FFT) } \\
\text { of reference waveform }\end{array}$ \\
\hline 10 & Repeat Steps 7-9 for each remaining reference waveform \\
\hline 11 & Compute reflection coefficient \\
\hline 12 & Plot and print data \\
\hline
\end{tabular}


conversion of the signal to a "zero-mean signal," which involves subtracting the average voltage of the entire waveform from each voltage within the waveform. This adjusts the mean of the waveform to zero to permit equivalent mathematical treatment of all data. Next, each binary value within the zero-mean waveform is converted to a true voltage value using a calculation based upon the ultrasonic receiver gain setting and the A/D board sensitivity setting. Then each voltage value within the waveform is squared and stored in a new array to compute the IBS value for the sample. The IBS value is determined by computing the mean of the 1089 squared voltage arrays. The TOF data from a single waveform is then computed using library routines provided by IDL, which finds the highest value within the string (the peak amplitude) and the location within the string that the peak occurs (the TOF value). The TOF values are then stored in a separate array. An FFT is also computed on a single waveform using an IDL library routine, and produces an array of real and complex values at each frequency. The magnitude at each frequency is then found by computing the absolute value of the complex number and storing the values in a separate array. The magnitude spectrum for all 1089 waveforms from a single sample are then averaged and stored in a new, one-dimensional array. Identical treatment of the $\mathrm{RF}$ waveform file from the reference sample is then performed. The reflection coefficient at each frequency is then determined by dividing the averaged magnitude array from the sample by the averaged magnitude array from the reference, and stored in a new one-dimensional array. Several items are then plotted, including a sample RF waveform, the averaged magnitude spectrum from both the sample and the reference, the reflection coefficient curve, and the TOF histograms of both the sample and the reference. A typical display produced by the program is shown in Figure 15.

Finally, a least-squares polynomial fit is performed on the reflection coefficient curve, yielding slope and Y- intercept values, and the variance on the TOF data is calculated, again by utilizing IDL library routines.

\section{Ultrasonic Data}

The IBS, reflection coefficient slope and Y-intercept, and TOF histogram variance data calculated by the IDL computer code are compiled in Table 3. Plots of selected squared and averaged waveform and TOF histogram data are presented in Appendix E.

\section{Destructive Analysis}

The strength of each bond specimen was determined by performing a uniaxial tensile test on all samples except sample numbers 6 and 9, which were cross-sectioned for metallurgical analysis, and sample number 1 , which broke during installation into the tensile testing machine. The tests were conducted using an Instron Model 1128 Universal Tester, using a cross-head speed of $0.15 \mathrm{inch} / \mathrm{min}$. The load at fracture and estimated tensile strength are listed in Table 3 . The stress was based on the measured bond line diameter after bonding. A problem that occurred, and was not considered in the design of the samples, was geometric distortion during the bonding process, specifically to the threaded sections. The thread diameter increased to varying degrees 

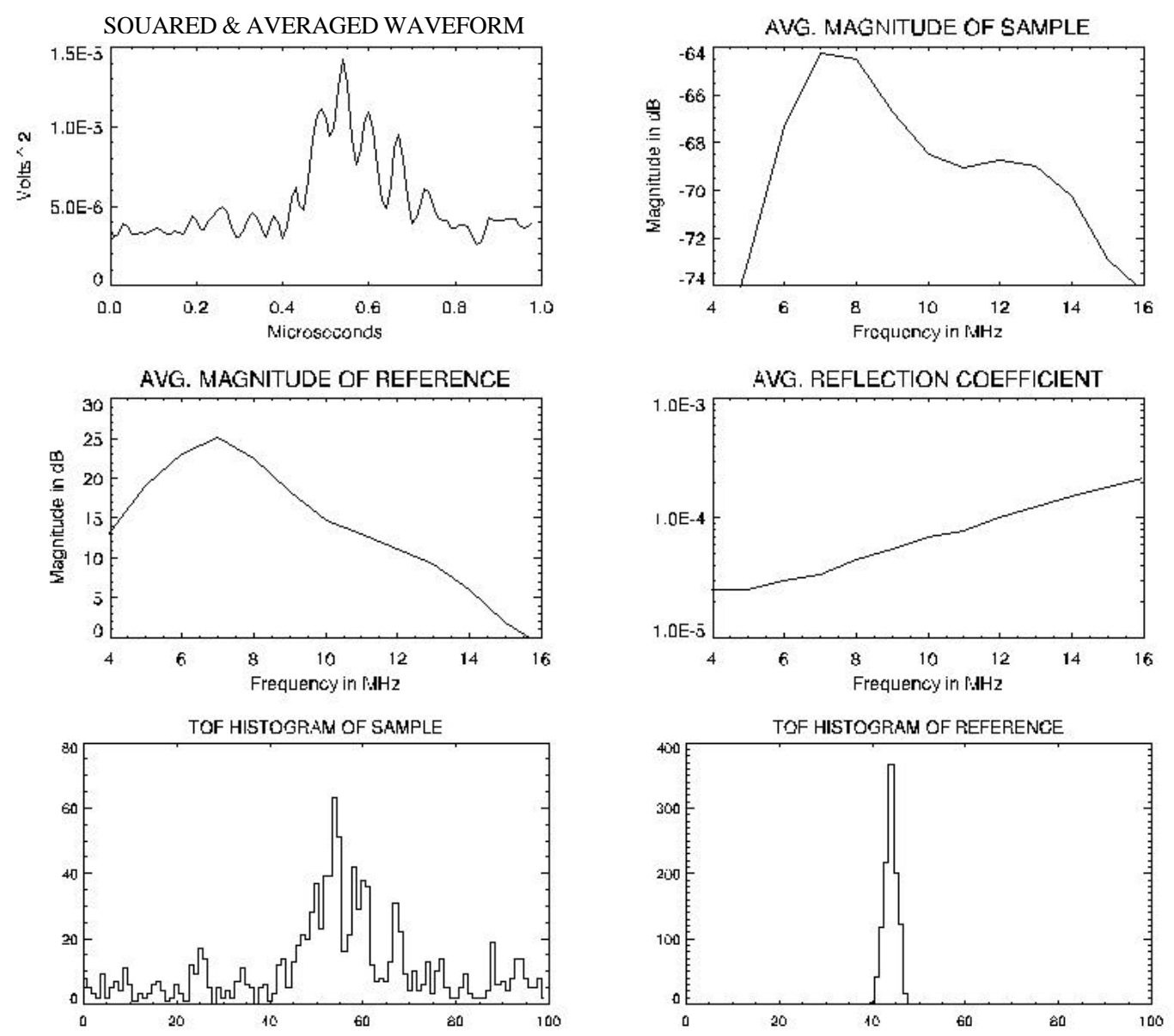

Figure 15. Graphical Output From IDL Computer Code

on all samples, causing difficulty when being threaded into the mating fixture on the tensile testing machine. Several samples could not be threaded into the fixture, and required re-threading. Sample 5 threaded into the fixture only partially, and during tensile testing the threads failed at a stress of $12,137 \mathrm{psi}$. The threads were subsequently repaired, and the sample later fractured at a stress of 11,731 psi, apparently suffering some damage during the initial test. The higher of the two values was listed for correlation.

\section{Optical Metallography of Bond Interface}

Samples \#6 and \#9 were moderate strength bonds chosen for destructive analysis and therefore not tensile tested. Each sample was axially cross-sectioned into four equal parts using a diamond cutting saw. Each face was polished, and photographs of the bond at several random locations at a magnification of 570x were taken. Each sample was then etched using a dilute nitric acid solution and photographed again in the same locations. Several examples are shown in Appendix F. Grain growth across the bond line is evident in several areas, and is consistent with the results reported by Eckman and Rosenblum (12,13). 
Table 3. Tensile Test Data and Calculated Acoustic Parameters

\begin{tabular}{|c|c|c|c|c|c|c|c|}
\hline Sample & $\frac{\text { Load @ }}{\text { Fracture }}$ & $\frac{\text { Stress@ }}{\text { Fracture }}$ & $\underline{\text { Side }}$ & IBS & $\frac{\text { TOF }}{\text { Variance }}$ & Slope & $\underset{\text { Intercept }}{\text { RC Y- }}$ \\
\hline & & (ksi) & & & & & \\
\hline \multirow[t]{2}{*}{1} & Note 1 & & $A$ & $1.29 \mathrm{E}-3$ & 2.53 & 2.51 & -3.05 \\
\hline & & & $B$ & $1.37 \mathrm{E}-3$ & 9.57 & 2.74 & -3.29 \\
\hline \multirow[t]{2}{*}{2} & 1417 & 7.230 & A & $3.17 \mathrm{E}-04$ & 2.58 & 2.850 & -3.71 \\
\hline & & 7.230 & B & $2.62 \mathrm{E}-04$ & 1.18 & 2.744 & -3.64 \\
\hline \multirow[t]{2}{*}{3} & 9037 & 46.107 & A & $6.75 \mathrm{E}-06$ & 54.05 & 1.501 & -3.26 \\
\hline & & 46.107 & B & 6.97 E-06 & 17.80 & 1.544 & -3.27 \\
\hline 4 & Note 2 & & & & & & \\
\hline \multirow[t]{2}{*}{5} & 2379 & 12.137 & $\bar{A}$ & $1.80 \mathrm{E}-05$ & 25.87 & 2.591 & -4.07 \\
\hline & & 12.137 & B & 1.57 E-05 & 4.02 & 2.431 & -3.95 \\
\hline \multirow[t]{2}{*}{6} & Note 3 & & A & $3.32 \mathrm{E}-4$ & 1.87 & 2.40 & -3.22 \\
\hline & & & B & 3.74 E-4 & 2.48 & 2.48 & -3.29 \\
\hline \multirow[t]{2}{*}{7} & 312 & 1.591 & $\bar{A}$ & $4.98 \mathrm{E}-04$ & 28.90 & 1.478 & -2.26 \\
\hline & & 1.591 & $\mathrm{~B}$ & $4.98 \mathrm{E}-04$ & 0.66 & 1.541 & -2.31 \\
\hline \multirow[t]{2}{*}{8} & 553 & 2.821 & $\bar{A}$ & $1.31 \mathrm{E}-05$ & 40.96 & 1.114 & -2.75 \\
\hline & & 2.821 & $B$ & $1.49 \mathrm{E}-05$ & 19.91 & 1.267 & -2.84 \\
\hline \multirow[t]{2}{*}{9} & Note 3 & & $A$ & $9.96 \mathrm{E}-06$ & 49.76 & 1.20 & -2.88 \\
\hline & & & B & $1.09 \mathrm{E}-05$ & 33.22 & 1.03 & -2.69 \\
\hline \multirow[t]{2}{*}{10} & 17734 & 90.479 & A & $4.08 \mathrm{E}-06$ & 467.62 & 1.851 & -3.69 \\
\hline & & 90.479 & B & 4.35 E-06 & 371.14 & 1.764 & -3.60 \\
\hline \multirow[t]{2}{*}{11} & 1454 & 7.418 & A & $2.19 \mathrm{E}-04$ & 26.29 & 1.931 & -2.87 \\
\hline & & 7.418 & $B$ & $2.17 \mathrm{E}-04$ & 1.20 & 1.977 & -2.91 \\
\hline \multirow[t]{2}{*}{12} & 11149 & 56.882 & $\mathrm{~A}$ & $3.47 \mathrm{E}-06$ & 705.51 & 1.723 & -3.62 \\
\hline & & 56.882 & $B$ & $3.98 \mathrm{E}-06$ & 819.91 & 1.833 & -3.68 \\
\hline \multirow[t]{2}{*}{13} & 17953 & 91.596 & A & $2.90 \mathrm{E}-06$ & 608.91 & 2.225 & -4.13 \\
\hline & & 91.596 & $B$ & $4.10 \mathrm{E}-06$ & 629.37 & 2.308 & -4.14 \\
\hline \multirow[t]{2}{*}{14} & 15513 & 79.148 & A & 3.10 E-06 & 827.82 & 2.326 & -4.22 \\
\hline & & 79.148 & $\bar{B}$ & $3.22 \mathrm{E}-06$ & 647.81 & 2.507 & -4.40 \\
\hline \multirow[t]{2}{*}{15} & 15907 & 81.158 & $\bar{A}$ & $3.04 \mathrm{E}-06$ & 1148.46 & 2.170 & -4.07 \\
\hline & & 81.158 & B & $3.46 \mathrm{E}-06$ & 682.61 & 2.596 & -4.47 \\
\hline \multirow[t]{2}{*}{16} & 3125 & 15.943 & A & 5.26 E-05 & 28.16 & 1.785 & -3.06 \\
\hline & & 15.943 & $B$ & $4.90 \mathrm{E}-05$ & 22.63 & 2.097 & -3.36 \\
\hline \multirow[t]{2}{*}{17} & 4667 & 23.811 & A & $2.18 \mathrm{E}-05$ & 28.50 & 1.774 & -3.22 \\
\hline & & 23.811 & $B$ & $1.55 \mathrm{E}-05$ & 6.92 & 1.875 & -3.39 \\
\hline \multirow[t]{2}{*}{18} & 4350 & 22.193 & $\bar{A}$ & $3.49 \mathrm{E}-05$ & 9.06 & 1.333 & -2.72 \\
\hline & & 22.193 & $B$ & $4.01 \mathrm{E}-05$ & 26.65 & 1.213 & -2.59 \\
\hline \multirow[t]{2}{*}{19} & 10732 & 54.755 & $A$ & $5.63 \mathrm{E}-06$ & 331.34 & 1.604 & -3.40 \\
\hline & & 54.755 & $B$ & $6.17 \mathrm{E}-06$ & 203.21 & 1.467 & -3.23 \\
\hline \multirow[t]{2}{*}{20} & 13383 & 68.159 & A & $5.54 \mathrm{E}-06$ & 134.66 & 1.503 & -3.28 \\
\hline & & 68.159 & B & $8.35 \mathrm{E}-06$ & 116.43 & 1.850 & -3.53 \\
\hline \multirow[t]{2}{*}{21} & 12102 & 61.636 & A & $5.40 \mathrm{E}-06$ & 293.58 & 1.952 & -3.73 \\
\hline & & 61.636 & $B$ & $4.32 \mathrm{E}-06$ & 418.74 & 1.837 & -3.67 \\
\hline 22 & 9451 & 48.132 & $\bar{A}$ & $4.63 \mathrm{E}-06$ & 127.25 & 1.682 & -3.49 \\
\hline & & 48.132 & B & $4.62 \mathrm{E}-06$ & 217.07 & 1.768 & -3.58 \\
\hline $\mathrm{S} 1$ & & & & $3.06 \mathrm{E}-06$ & 734.668 & 2.119 & -4.01 \\
\hline & & & & & & & \\
\hline Half & & & & $4.08 \mathrm{E}-02$ & 1.84 & 0.00 & 0.00 \\
\hline
\end{tabular}


The fracture surfaces of samples \#2, \#8, \#10, \#17, and \#19 were analyzed using a JEOL Model JSM IC848 scanning electron microscope (SEM). Digital images are shown in Appendix F.

\section{$\underline{\text { Results }}$}

The logarithm of the IBS values, the TOF variance, and the reflection coefficient slope and Y-intercept are each plotted against tensile strength and are shown in Figures 16, 17, 20, and 21, respectively. Trendlines are calculated using the least squares method, with a power curve being applied to the log IBS data, linear regression curves to the reflection coefficient slope and Y-intercept data, and a logarithmic curve to the TOF variance data. The $\mathrm{R}^{2}$ values are also listed, showing an indication of the quality of the fit of the trendlines to the data. The IBS and TOF variance methods clearly provided the best correlation with bond strength, with values of 0.714 and 0.733 , respectively. If the data from sample \#5, which was likely damaged during tensile testing as discussed in 4.5.3, is not considered in the calculations, the $\mathrm{R}^{2}$ values remain essentially unchanged with the exception of the reflection coefficient Y-intercept fit, which increases from 0.474 to 0.632 . A comparison of all $\mathrm{R}^{2}$ values, with and without considering sample \#5, is shown in Table 4. Note that pairs of data points are plotted corresponding to data acquired from sides A and B. However, data points within each pair are being considered independently for this project. Calculations from both sides of each sample always result in some difference, the greatest being in the TOF variance. One could easily argue that the data from the side that predicts the lowest bond strength is the best choice. If the least squares method is applied to only the IBS and TOF variance data from the side that would represent a correspondingly weaker bond, the $\mathrm{R}^{2}$ values are 0.710 and 0.810 , respectively, which are not significantly different from the values calculated from the entire data set. Having such a limited sample size, however, it is difficult to draw conclusions about the side-to-side variability.

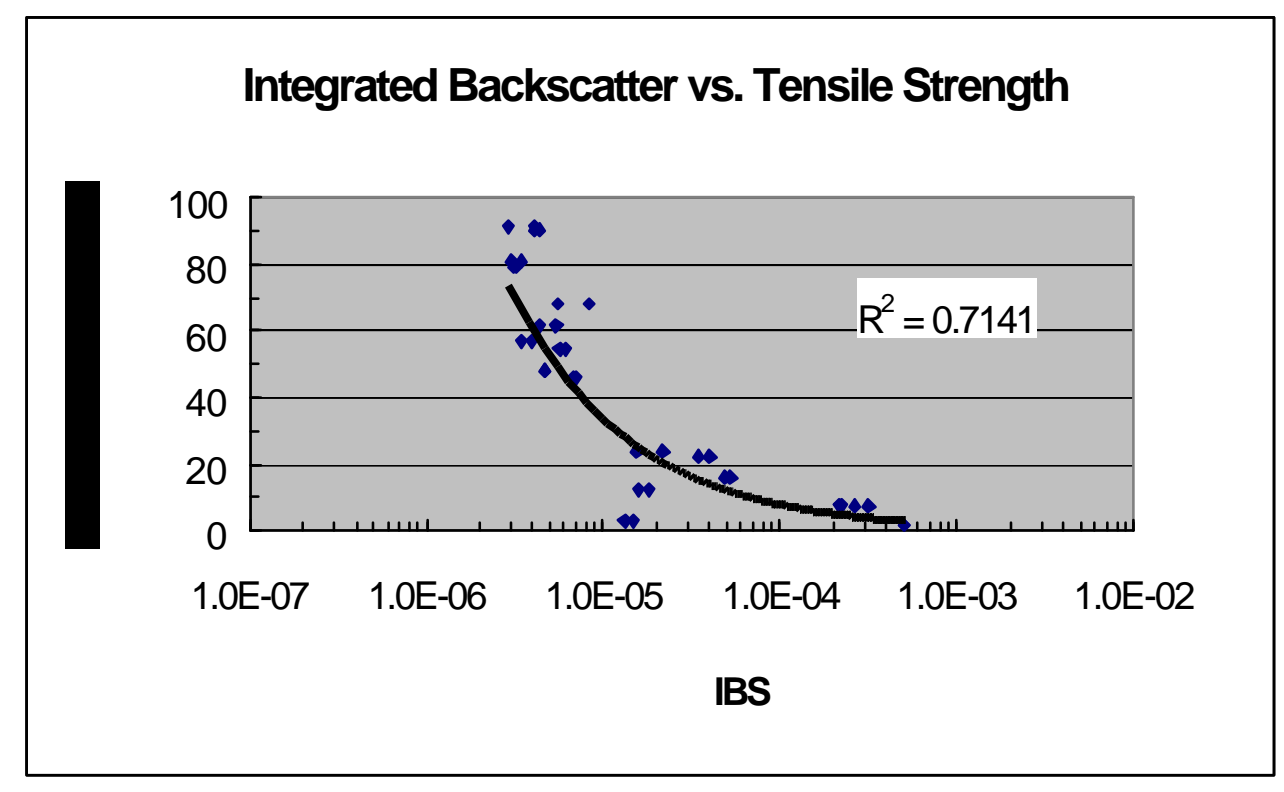

Figure 16. Integrated Backscatter Versus Tensile Strength 


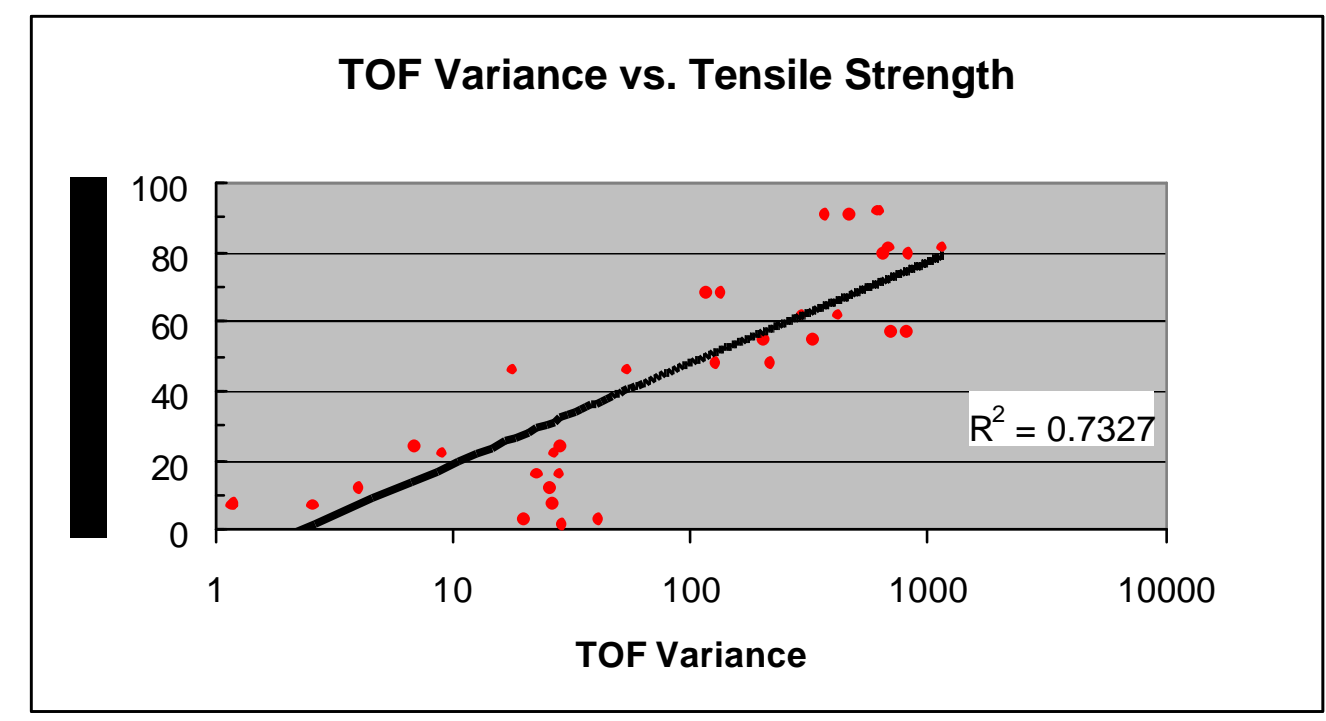

Figure 17. Time-of-Flight Variance Versus Tensile Strength

Table 4. Regression Analysis $\mathbf{R}^{2}$ Values

\begin{tabular}{|c|c|c|c|}
\hline Method & All Samples & W/O Sample \#5 & W/O Sample \#5 \& \#8 \\
\hline IBS & 0.714 & 0.726 & 0.934 \\
\hline TOF Variance & 0.733 & 0.728 & 0.762 \\
\hline R.C. Y-Intercept & 0.456 & 0.613 & 0.580 \\
\hline R.C. Slope & 0.030 & 0.080 & 0.030 \\
\hline
\end{tabular}

\section{Bond Strength Versus Integrated Backscatter and TOF Variance}

The IBS method easily discriminates between low strength bonds (less than $30 \mathrm{ksi}$ ) and moderate to high strength bonds (45 ksi and greater). Ojard, et. al, reported that as the bond strength drops from the highest value achieved to approximately half the maximum strength in copper/copper bonds, the IBS value increased by a factor of five (9). The beryllium-copper bond data did not exhibit this sensitivity; however, Ojard's data was based on a limited sample set of only four. The TOF variance data showed a similar ability in distinguishing low strength from high strength bonds.

Review of the IBS data reveals that sample \#8 is the only data point that has a significant deviation from the curve. The IBS and TOF calculations both predicted a moderate strength bond of approximately $30 \mathrm{ksi}$; however, it failed at only $2.8 \mathrm{ksi}$. Reviewing the c-scan data from sample \#8, shown in Figure 18, shows a gradient in the acoustic response, varying from a nearly indistinguishable bond line in the lower right corner, to an easily distinguished bond line in the 


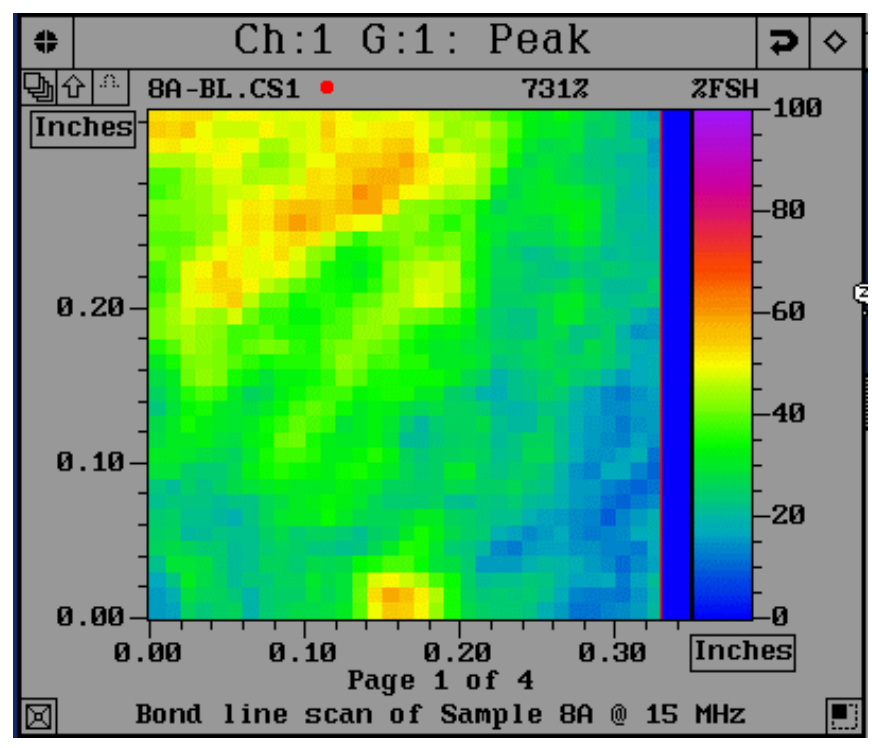

Figure 18. C-scan Image of Sample \#8

upper left corner, averaging $12 \mathrm{~dB}$ above the grain noise. If the gradient continued to each edge of the specimen, this would increase the probability of the crack initiation site to occur at the edge having the strong acoustic response, and cause a different mode of fracture than if the bond quality was uniformly distributed. The surface of sample \#8 was reviewed using the SEM, and a crack initiation site was not observed. However its surface texture was significantly different than sample \#19, a moderate strength bond exhibiting the classic cuplet appearance due to the void sheet fracture mechanism, and sample \#2, a weak bond which failed at $7.2 \mathrm{ksi}$ and exhibits no evidence of fracture. Sample \#8 exhibits both ductile and brittle fracture characteristics, suggesting a different fracture mechanism occurred, perhaps by cleavage. Samples \#2, \#8, and \#19 are shown in Appendix F for comparison. No other c-scan revealed this type of acoustic gradient. If this data point is not considered due to the suspected difference in fracture mechanism, the $\mathrm{R}^{2}$ value of the IBS data dramatically improves to 0.934 . All $\mathrm{R}^{2}$ values without sample \# 5 and \#8 are shown in Table 4. The IBS and TOF histogram techniques did yield dramatically different results on sample \#3, sample \#12, sample \#19, and sample \# 22, which had similar tensile strength data (46 ksi, $56 \mathrm{ksi}, 54 \mathrm{ksi}$, and $48 \mathrm{ksi}$, respectively). The IBS values differed by approximately a factor of 1.5 , whereas the TOF variance values differed by a factor of approximately 20. Viewing the peak amplitude c-scan data from the four samples, shown in Figure 19, highlights how their amplitude data and relative spatial distributions differ. Sample \#12 has a homogeneous distribution with little variation in amplitude. The other samples have varying numbers of localized areas where the reflected signal from the bond has increased from $2 \mathrm{~dB}$ (decibels) to $6 \mathrm{~dB}$ above the grain noise. The data indicates that the strength of these samples was not significantly affected by these localized areas of incomplete bond, suggesting an apparent advantage the IBS technique has in averaging the total reflected energy over the bond area. The TOF variance technique considers only the number of occurrences at the bond line and not the amplitude of each occurrence, suggesting that a large error may be produced for bond strengths in the intermediate range. 




Sample 3

Sample 22

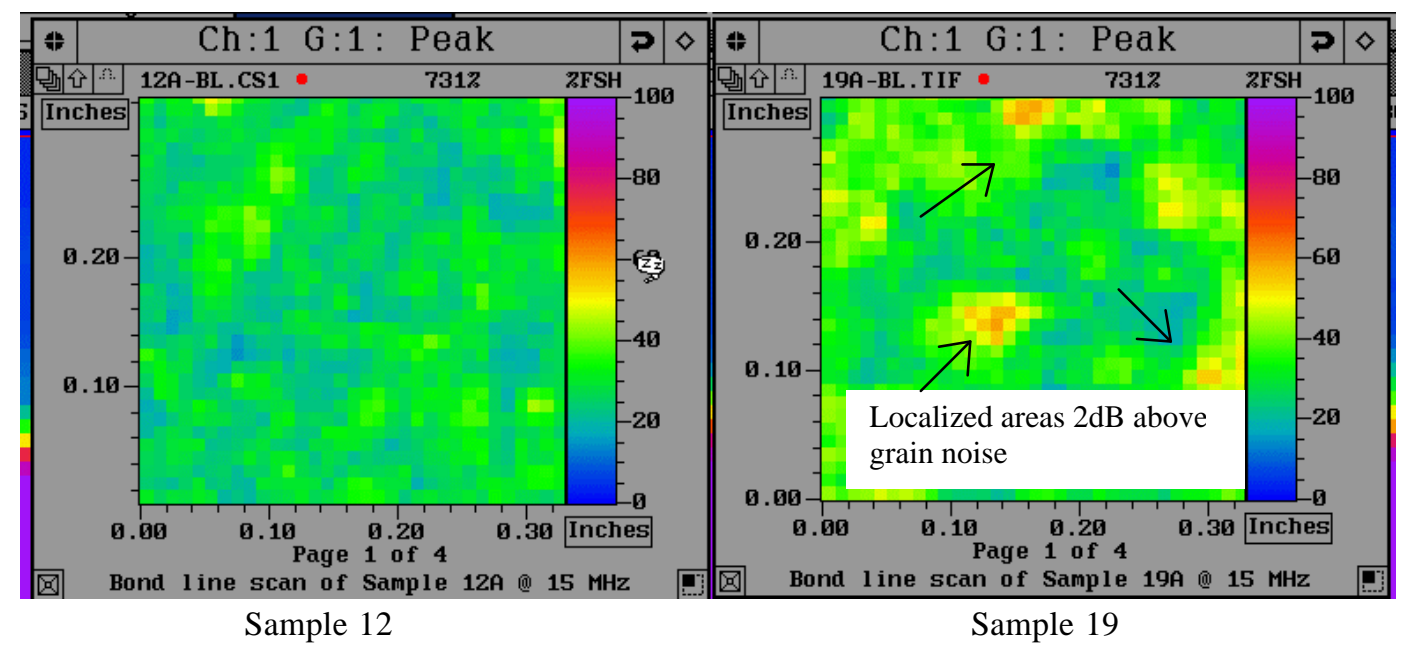

Figure 19. C-scan Images of Samples \#3, \#12, \#19, and \#22

\section{Bond Strength Versus Frequency Domain Reflection Coefficient Slope and Y-Intercept Data}

Perhaps the most surprising data was yielded by the reflection coefficient slope and Y-intercept correlations. Initial evaluation of the reflection coefficient slope data shown in Figure 20 reveals a very poor correlation to bond strength. The intent of the reflection coefficient method is to compare a poor bond (half sample), which is frequency independent, with good quality bond, which is predicted to show frequency dependence, with the result being most sensitive to changes at higher frequencies. Regarding the reflection coefficient slope data, if only the samples having a tensile strength of less than $40 \mathrm{ksi}$ are considered, there is considerable scatter and no apparent correlation with bond strength. However, if only the bond strengths above $40 \mathrm{ksi}$ are considered, the data does exhibit some correlation, where bonds in the $40 \mathrm{ksi}-60 \mathrm{ksi}$ range have slopes of approximately 1.6, and bond strengths over $80 \mathrm{ksi}$ have slopes near 2.2. 
The dashed line in Figure 20 represents the least squares fit of only the high strength data, which results in an improved $\mathrm{R}^{2}$ value of 0.505 . This observation appears to agree with the aforementioned theory of the reflection coefficient, which states that poor bonds are frequency independent. The bonds weaker than $40 \mathrm{ksi}$ produce a significant reflected signal, which is likely frequency independent. The ratio of the magnitude spectra of signal from a weak bond with that of a reference signal, which is also frequency independent, will result in a reflection coefficient that is also frequency independent. The bonds having strengths over $40 \mathrm{ksi}$, however, begin to display the frequency dependence of signals reflected from good bond, which results in an increase of the reflection coefficient with higher frequencies.

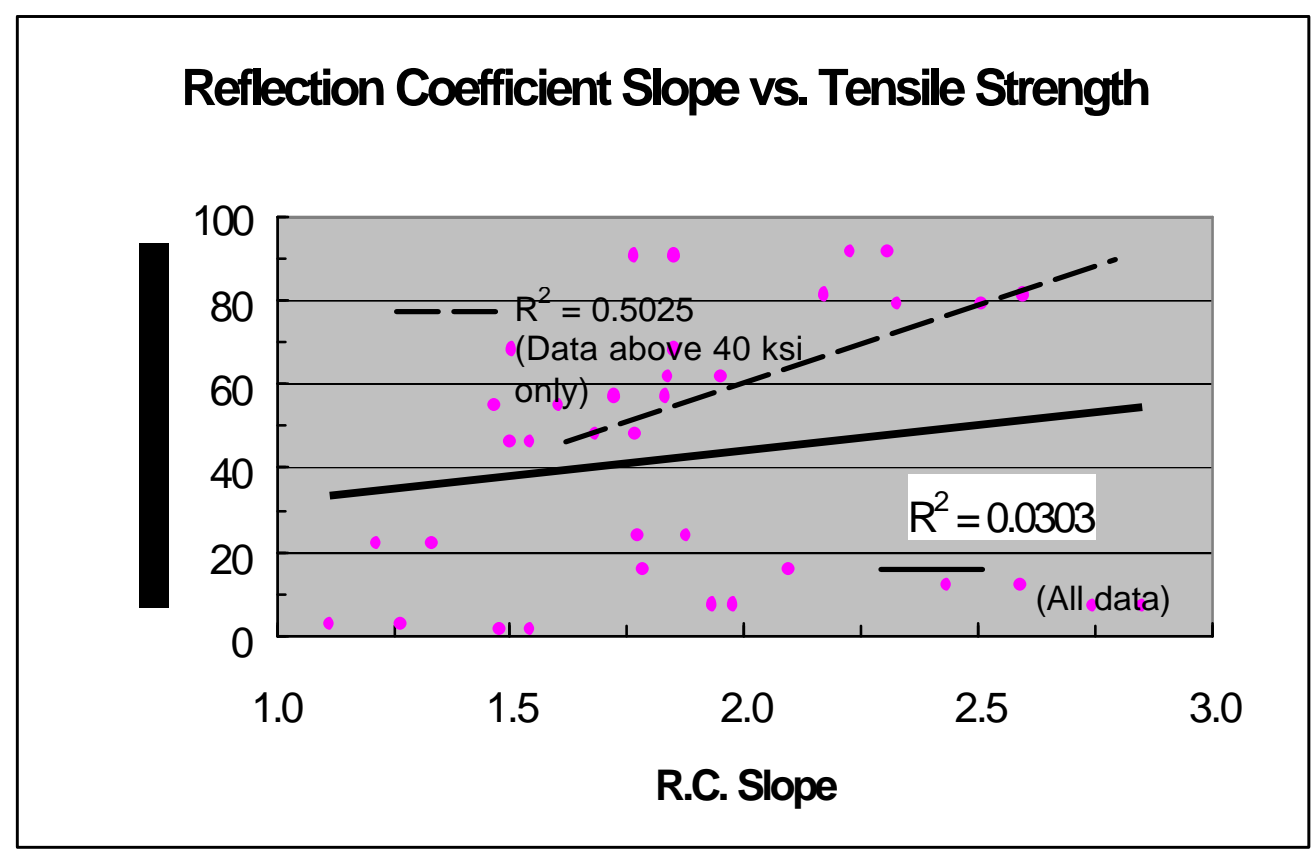

Figure 20. Reflection Coefficient Slope Versus Tensile Strength

The reflection coefficient $\mathrm{Y}$-intercept data shows only a moderate ability to distinguish between low strength, moderate strength ( $40 \mathrm{ksi}-60 \mathrm{ksi}$ ), and high strength bonds as shown in Figure 21 . This technique appears to be predominantly a function of the ratio of the magnitudes at each frequency of the signals from the sample and reference, and is affected minimally by the slope of the curve. 


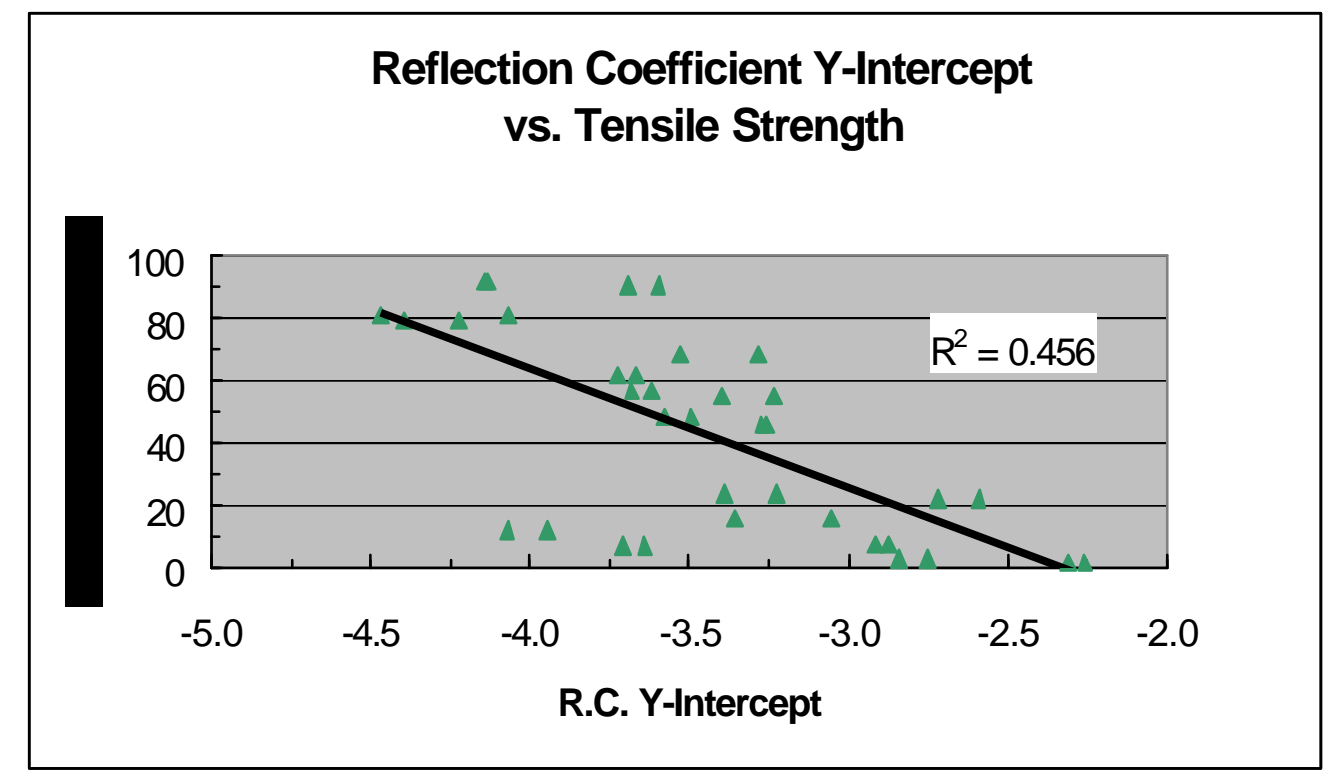

Figure 21. Reflection Coefficient Y-Intercept Versus Tensile Strength

\section{Accomplishments}

Several ultrasonic techniques, including integrated backscatter, time-of-flight histogram, and frequency domain reflection coefficients slope and Y-intercept, were compared in their ability to characterize the strength of a series of beryllium-copper/beryllium-copper diffusion bonds. Enhancements were introduced on two techniques in an attempt to provide quantitative analysis which had not been reported to date. The first enhancement was the calculation of the variance in the TOF histogram technique to provide a quantitative method of analysis. The second was the utilization of the slope and Y-intercept of the frequency domain reflection coefficient curves. The analysis concluded that the integrated backscatter technique and time-of-flight histogram variance provided very good correlation, and the Y-intercept of the reflection coefficient technique provided moderate correlation with the full range of bond strengths. The reflection coefficient slope technique appears to show some correlation only with bonds having strengths of over $40 \mathrm{ksi}$, but is of little use without additional knowledge to determine which strength distribution the specimen belongs in. The IBS and TOF variance techniques clearly resulted in the best fit with this. Though the time-of-flight histogram variance technique showed a good correlation to bond strength, there is evidence that it may produce a wide error on bonds where small isolated areas of poor bond are distributed within an otherwise well-bonded interface. The IBS technique, which accounts for amplitude information, appears to overcome this condition.

Finally, the importance of considering the spatial distribution of acoustic data is recognized in the evaluation of data produced by the four techniques. In several instances, evaluation of c-scan plots was necessary to explain specific behavior. 


\section{Future Work}

Based on the results of this work, the following are recommended:

1. Additional samples of diffusion bonded beryllium-copper should be produced and analyzed using the ultrasonic techniques presented in this work to provide a stronger statistical sample.

2. Solid state bonds of different alloys should be investigated using the ultrasonic techniques presented in this work and compared with the beryllium-copper results. This would determine which techniques are robust and which have possible limitations due to specific material characteristics. 


\section{References}

1. R. E. Reed-Hill, Physical Metallurgy Principles, $2^{\text {nd }}$ Edition, D. Van Nostrand Co., 1973.

2. R. Bruce Thompson, "Theory and Application of Ultrasonic Microstructural Characterization," Journal of Metals, October 1992.

3. American Society for Nondestructive Testing, Nondestructive Testing Handbook, $2^{\text {nd }}$ Edition, Volume 7, "Ultrasonic Testing," 1991.

4. G. C. Ojard, O. Buck, D. K. Rehbein, and A. Bevolo, "Bond Strength Evaluation in Dissimilar Materials," Review of Progress in Quantitative Nondestructive Evaluation, Vol. 10B, 1991.

5. G. H. Thomas and J. R. Spingarn, "Effects of Microstructure and Bond Quality on the Ultrasonic Evaluation of Dissimilar Metal Friction Welds," Review of Progress in Quantitative Nondestructive Evaluation, Vol. 9, 1990.

6. G. H. Thomas and J. R. Spingarn, "Ultrasonic Determination of Diffusion-Bond Strength," Review of Progress in Quantitative Nondestructive Evaluation, Vol. 3B, 1984, pp. 12431250.

7. O. Buck, D. K. Rehbein, R. B. Thompson, D. D. Palmer, and J. J. H. Brasche, "Nondestructive Characterization and Bond Strength of Solid-Solid Bonds," Review of Progress in Quantitative Nondestructive Evaluation, Vol. 8B, 1988.

8. H. Kate and S. Abe, "Ultrasonic Evaluation of the Bonding Strength of Dissimilar Metal Bonds,” NDT\&E International, Vol. 29, No. 6, pp. 355-361, 1996.

9. G. C. Ojard, O. Buck, and D. K. Rehbein, "Strength, Ultrasonic and Metallurgical Evaluation of Diffusion Bonds," Ultrasonic Characterization of Mechanics and Interfaces, ASME 1993.

10. E. J. Nieters, M. F. X. Gigliotti, L. C. Perocchi, and R. S. Gilmore, "Ultrasonic Evaluation of Titanium Alloy Diffusion Bonds, "Review of Progress in Quantitative Nondestructive Evaluation, Vol. 15, 1996.

11. D. D. Palmer, C. D. Roberts, D. K. Rehbein, J. F. Smith, and O. Buck, "Strength and Ultrasonic Characterization of Metallic Interfaces," Review of Progress in Quantitative Nondestructive Evaluation, Vol. 7B, 1988.

12. D. C. Eckman, Diffusion Bonding of Beryllium Copper Alloys, a thesis presented to the Faculty of the Graduate School, University of Missouri - Columbia, 1991.

13. D. C. Eckman and B. Z. Rosenblum, Diffusion Bonding of Beryllium Copper Alloys, Honeywell Federal Manufacturing \& Technologies: KCP-613-4321, September 1990. 


\section{Acknowledgments}

The author wishes to gratefully acknowledge Steven P. Neal, Ph.D., Professor of Mechanical and Aerospace Engineering, University of Missouri, Columbia, and Ben-Zion Rosenblum, Ph.D., Honeywell Federal Manufacturing \& Technologies, for their significant technical contributions to this project and their continuing inspiration, without which this work would not have been possible. Additionally, the author wishes to thank Robert E. Williams, Ph.D., Edward Wenski, Wayne McLarren, Arthur Bundy, and Robert Jump, all of Honeywell Federal Manufacturing \& Technologies, for their technical assistance. 


\section{Appendix A}

Sample Design 


\section{$\underline{\text { Appendix A }}$}
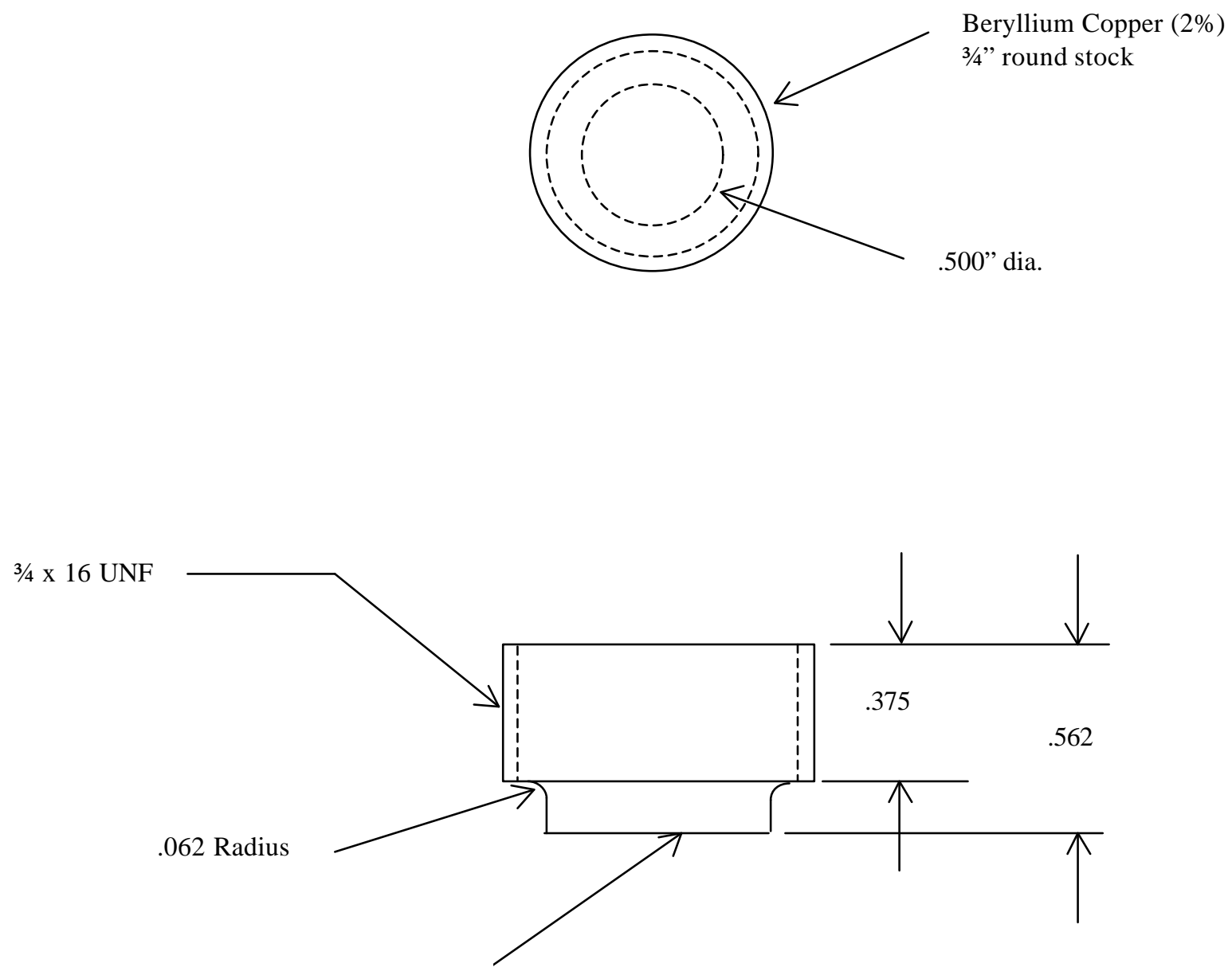

Flat to $0.0005 "$ or better

$0.80 \sqrt{ }$

Figure A1: Beryllium-Copper Diffusion Bond Sample Design 


\section{Appendix B}

\section{Diffusion Bond Equipment}




\section{Appendix B}

Table B1. Equipment List for Beryllium-Copper Sample Preparation and Diffusion Bond Procedure

\begin{tabular}{|c|c|c|}
\hline Process & Equipment & Manufacturer \\
\hline polishing/lapping & $\begin{array}{c}\text { METADI Diamond } \\
\text { Suspension }-15,9,6 \text {, and } 3 \\
\text { micron } \\
\end{array}$ & Buehler Ltd. \\
\hline & surface plates & Honeywell FM\&T \\
\hline $\begin{array}{l}\text { surface profile } \\
\text { analysis } \\
\end{array}$ & $\begin{array}{c}\text { TENCOR Surface Profiler } \\
\text { Model P-11 }\end{array}$ & Tencor Inc. \\
\hline etching & $\begin{array}{c}\text { sulfuric acid } \\
\text { nitric acid } \\
\text { deionized water } \\
\end{array}$ & \\
\hline diffusion bonding & $\begin{array}{l}\text { vacuum chamber } \\
\text { Model KHF-2136 }\end{array}$ & K.H. Frederick Co. \\
\hline & $\begin{array}{c}\text { mechanical roughing pump } \\
\text { Model SD-1400 }\end{array}$ & Varian Inc. \\
\hline & $\begin{array}{l}\text { cryogenic high vacuum pump } \\
\text { Model 313-0100 }\end{array}$ & Varian Inc. \\
\hline & $\begin{array}{c}\text { power generator } \\
\text { Model } 3200\end{array}$ & $\begin{array}{l}\text { ENI Power } \\
\text { Systems }\end{array}$ \\
\hline & impedance matching network & Honeywell FM\&T \\
\hline
\end{tabular}




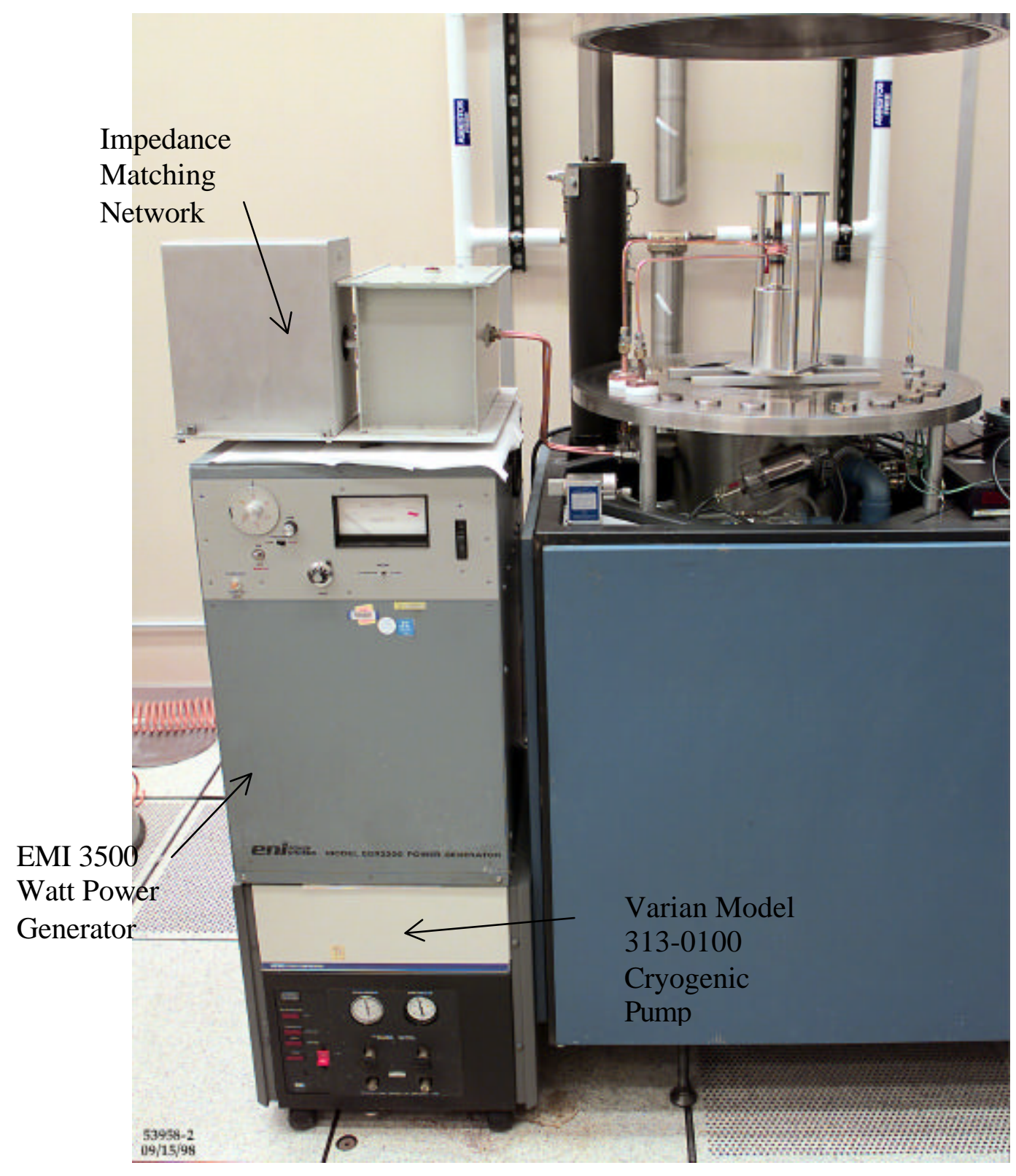

Figure B1. Diffusion Bond System and Fixturing 




Figure B2. Diffusion Bond Fixture 


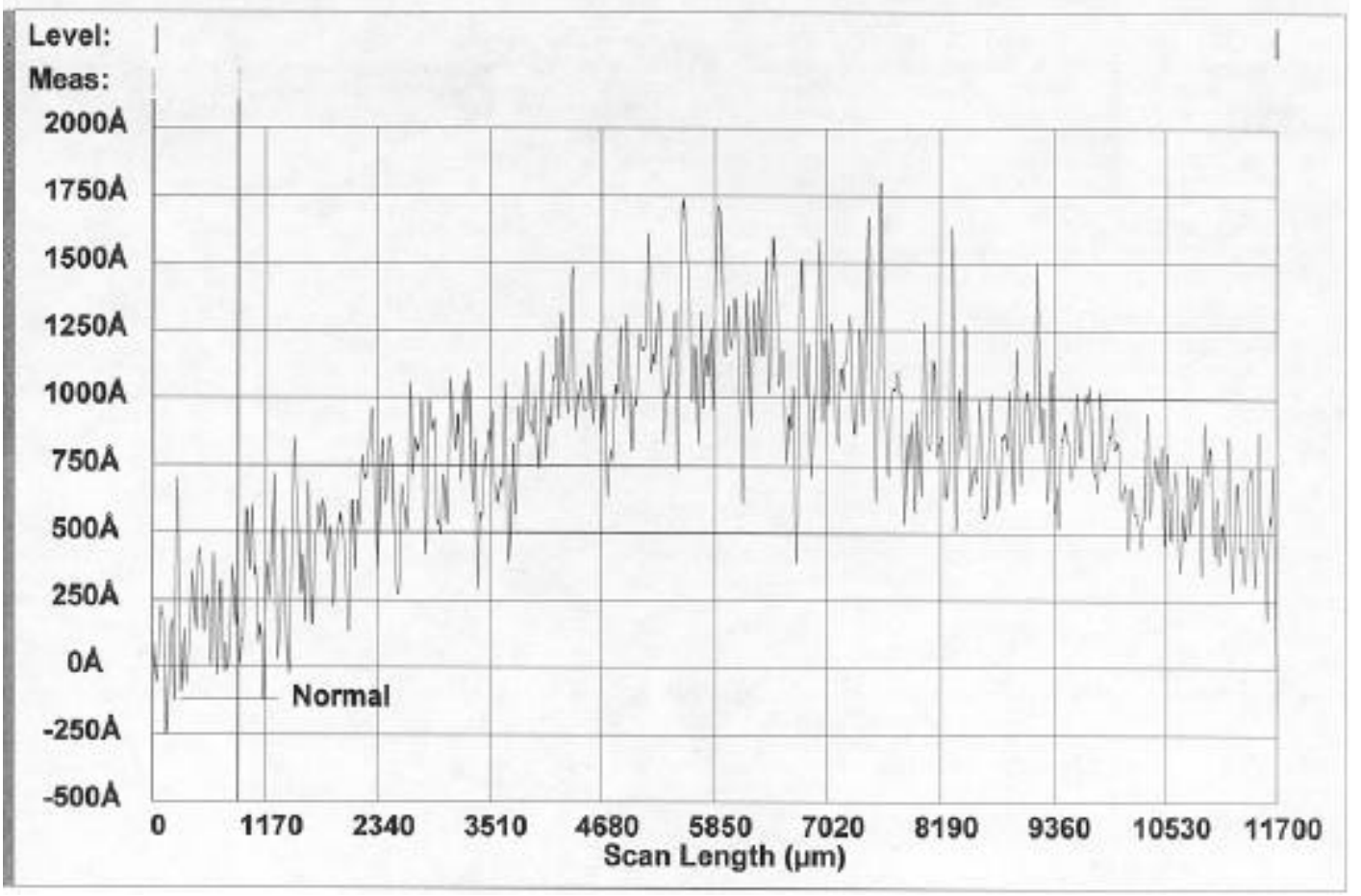

Figure B3. Pre-etch Surface Profile Measurement on Sample 12B



Figure B4. Post-etch Surface Profile Measurement on Sample 12B 


\section{Appendix C}

\section{Diffusion Bond Parameters}




\section{Appendix C}

Table C1. Diffusion Bond Parameters

\begin{tabular}{|c|c|c|c|}
\hline Sample \# & $\begin{array}{c}\text { Average Temp. } \\
\left({ }^{\circ} \mathrm{C}\right)\end{array}$ & $\begin{array}{c}\text { Time @ Max. Temp. } \\
\text { (min.) }\end{array}$ & $\begin{array}{c}\text { Nominal } \\
\text { Load } \\
\text { (psi) }\end{array}$ \\
\hline 1 & 750 & 60 & 500 \\
\hline 2 & 700 & 120 & 500 \\
\hline 3 & 800 & 180 & 500 \\
\hline 4 & 800 & 120 & 500 \\
\hline 5 & 750 & 60 & 1000 \\
\hline 6 & 800 & 30 & 500 \\
\hline 7 & 800 & 180 & 100 \\
\hline 8 & 800 & 120 & 500 \\
\hline 9 & 800 & 180 & 500 \\
\hline 10 & 800 & 180 & 500 \\
\hline 11 & 800 & 180 & 500 \\
\hline 12 & 800 & 180 & 500 \\
\hline 13 & 800 & 240 & 500 \\
\hline 14 & 800 & 480 & 500 \\
\hline 15 & 800 & 360 & 500 \\
\hline 16 & 800 & 525 & 80 \\
\hline 17 & 800 & 480 & $500^{*}$ \\
\hline 18 & 800 & 105 & 500 \\
\hline 19 & 800 & 180 & 500 \\
\hline 20 & 800 & 180 & 500 \\
\hline 21 & 800 & 180 & 500 \\
\hline 22 & 800 & 240 & 500 \\
\hline 23 & 800 & 180 & 500 \\
\hline 24 & 800 & 180 & 500 \\
\hline
\end{tabular}

* - load removed after 60 minutes 
Appendix D

Computer Code 


\section{Appendix D}

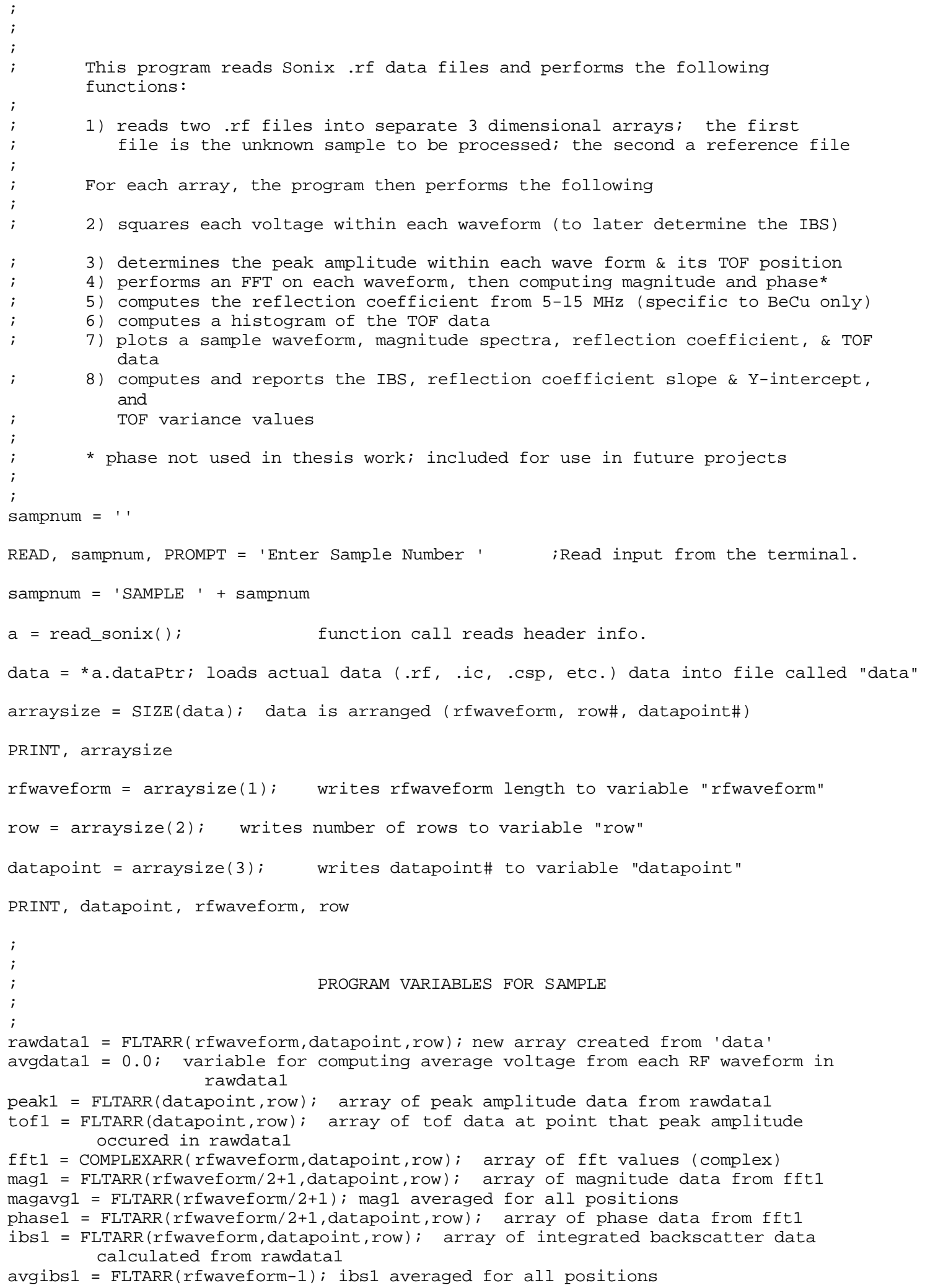




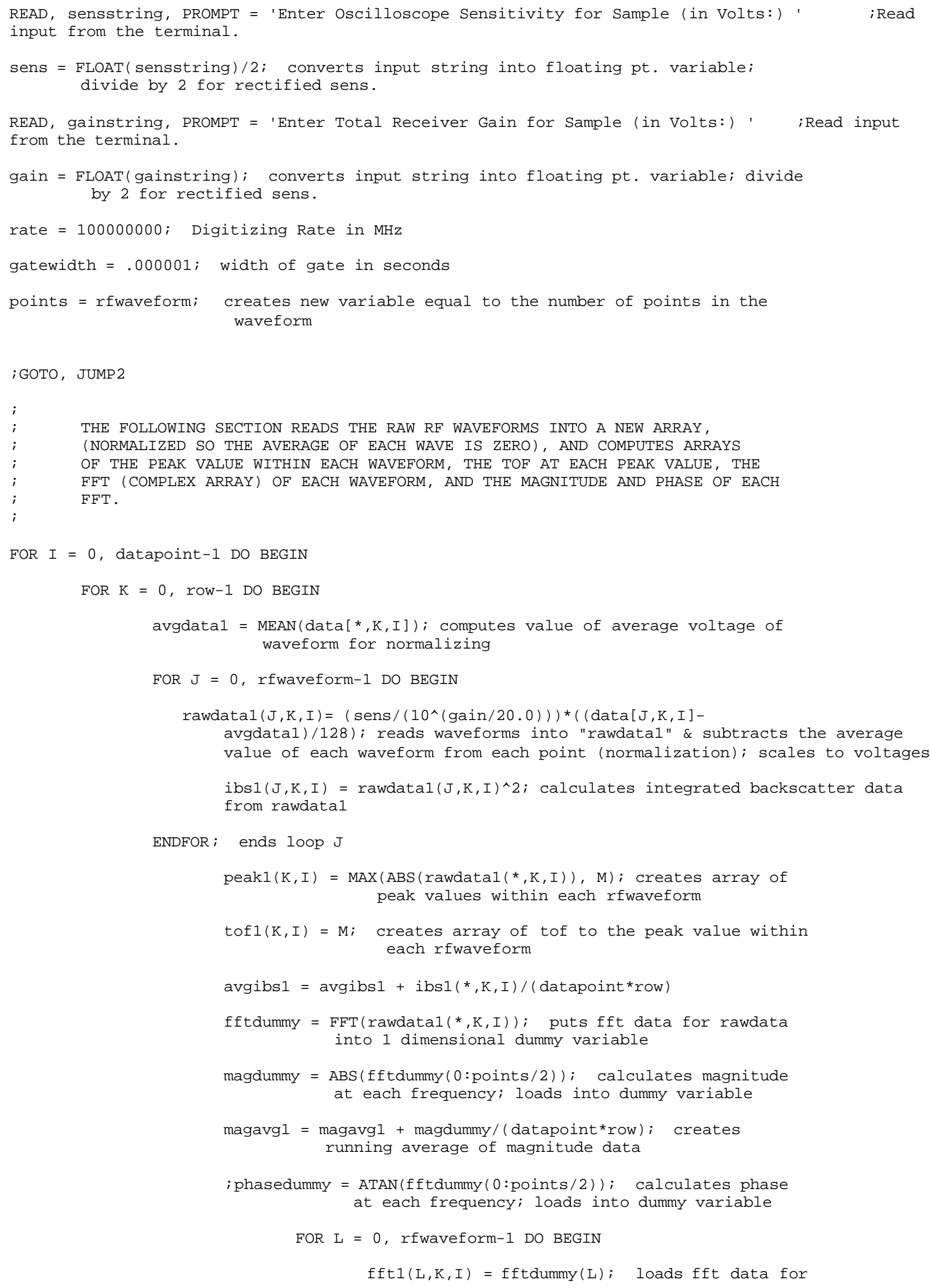


rawdata into 3d array fft 1

(fftdata, row, datapoint)

ENDFOR; ends loop L

FOR $M=0$, rfwaveform/2 DO BEGIN

$\operatorname{mag} 1(\mathrm{M}, \mathrm{K}, \mathrm{I})=$ magdummy $(\mathrm{M})+.0000001 ;$ creates $3 d$ array of mag. data from fft 1

; phase1 $(\mathrm{M}, \mathrm{K}, \mathrm{I})$ = phasedummy $(\mathrm{M})$ *!RADEG; creates

3darray of phase data from

fftlconverted to degrees

ENDFOR; ends loop M

ENDFOR; ends loop $\mathrm{K}$

ENDFOR; ends loop I

;

;

;

;

i

;

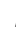

; ansstring $=$ ''

; READ, ansstring, PROMPT = 'Read in a reference RF waveform? (yes or no): input from the terminal.

; IF (STRUPCASE (ansstring) EQ 'NO') THEN GOTO, JUMP1

$\mathrm{b}=$ read_sonix ();

LANL function call reads header info.

datab $=\star b \cdot$ dataPtr;

loads actual data (.rf, .ic, .csp, etc.) data into file called "data"

i

;

rawdata2 = FLTARR (rfwaveform, datapoint, row); new array created from 'data'

avgdata $2=0.0$; variable for computing average voltage from each $\mathrm{RF}$ waveform in rawdata1

peak2 = FLTARR (datapoint, row); array of peak amplitude data from rawdatal

tof2 = FLTARR (datapoint, row); array of tof data at point that peak amplitude occured in rawdata2

fft2 = COMPLEXARR (rfwaveform, datapoint, row); array of fft values (complex)

mag2 = FLTARR (rfwaveform/2+1, datapoint, row); array of magnitude data from fft2

magavg2 = FLTARR (rfwaveform/2+1); mag2 averaged for all positions

phase2 = FLTARR (rfwaveform/2+1, datapoint, row); array of phase data from fft2

ibs2 = FLTARR (rfwaveform, datapoint, row); array of integrated backscatter data from fft 2

avgibs2 = FLTARR (rfwaveform-1); ibs2 averaged for all positions

reflcoef = FLTARR (rfwaveform/2+1, datapoint, row); array of frequency dependent reflectioncoefficients

; READ, sensstring, PROMPT = 'Enter Oscilloscope Sensitivity for Reference (in Volts:) '; Read input from the terminal.

; sens2 = FLOAT (sensstring); converts input string into floating pt. variable ;READ, gain2string, PROMPT = 'Enter Total Receiver Gain for Sample (in Volts:) ' ;Read input from the terminal.

; gain2 = FLOAT (gainstring)/2; converts input string into floating pt. variable; divide by 2 for rectified sens.

sens $2=2.0$ 


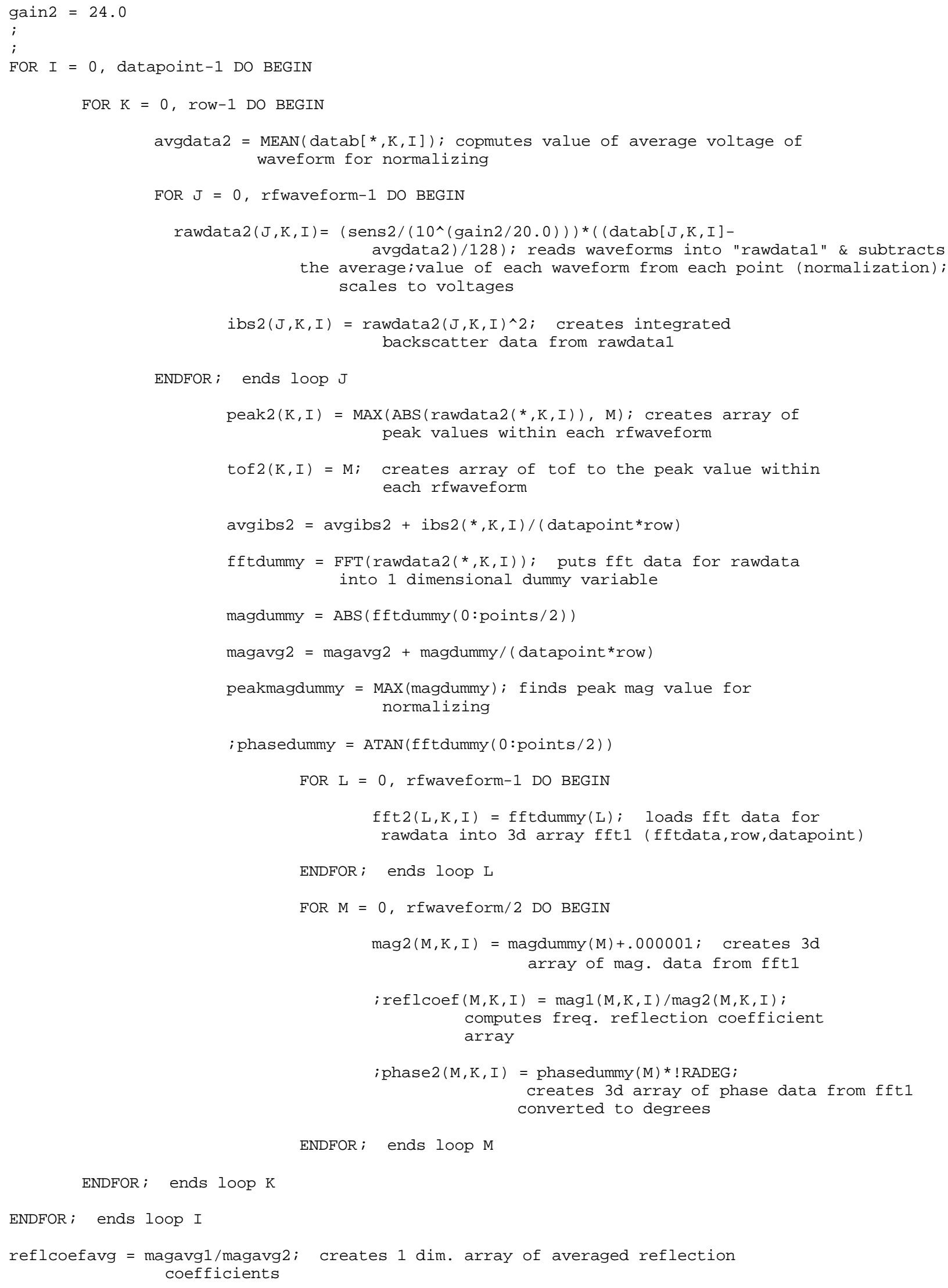




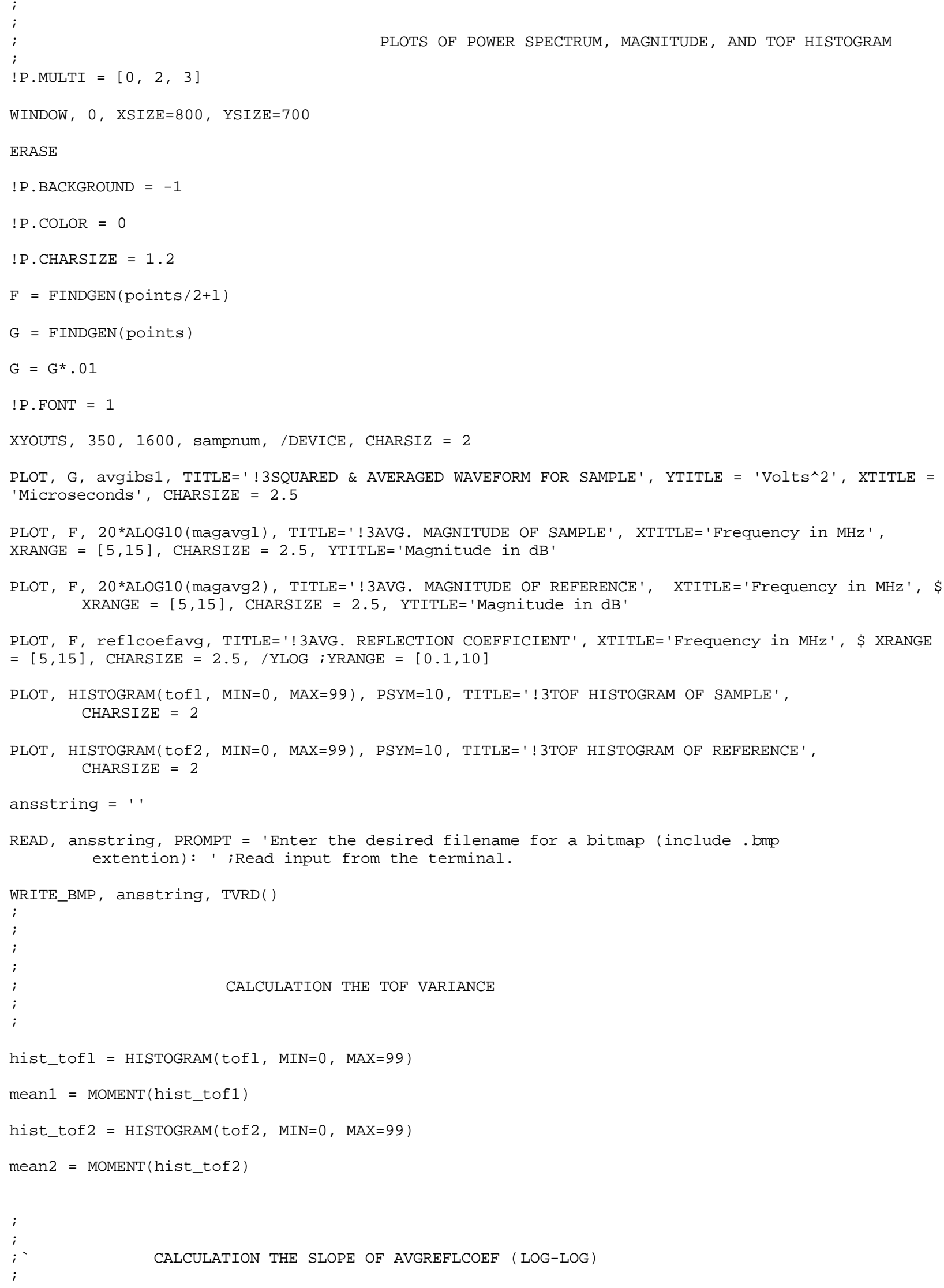




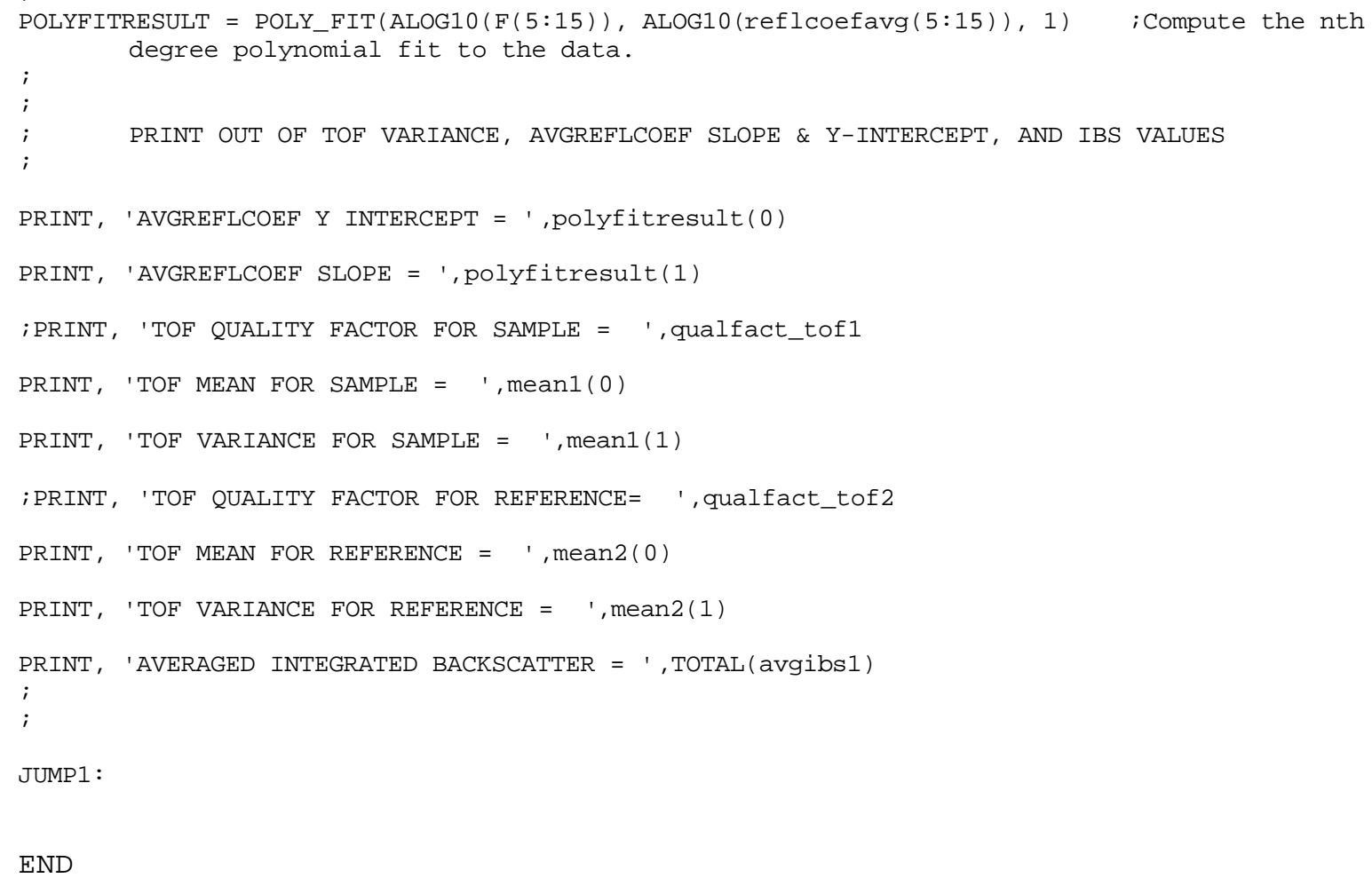




\section{Appendix E \\ Reflection Coefficient and TOF Histogram Plots}




\section{APPENDIX E}

Squared \& Averaged Waveform for Sample \#2A



Squared \& Averaged Waveform for Sample \#2B

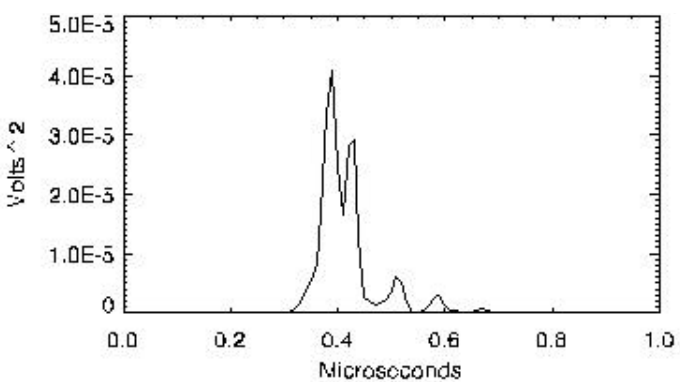

Squared \& Averaged Waveform for Sample \#8A

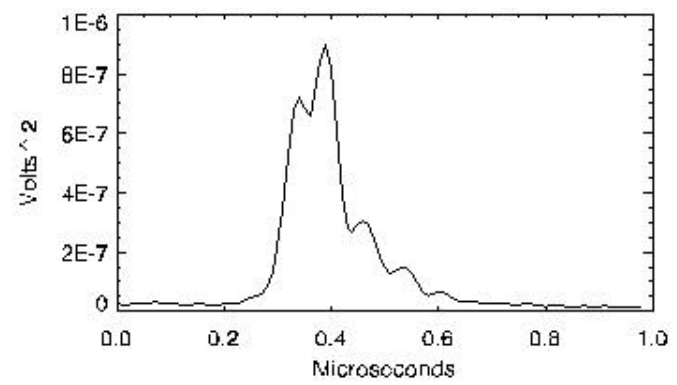

Squared \& Averaged Waveform for Sample \#8B



TOF Histogram of Sample \#2A


TOF Histogram of Sample \#8B

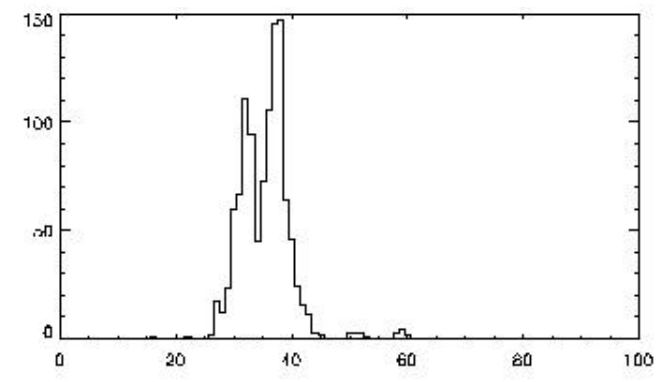


Squared \& Averaged Waveform for Sample \#10A

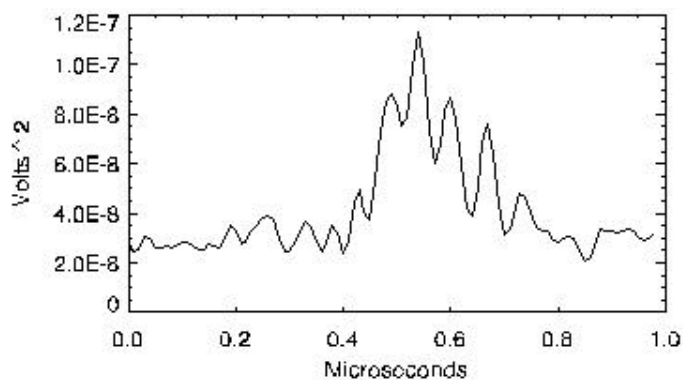

Squared \& Averaged Waveform for Sample \#10B

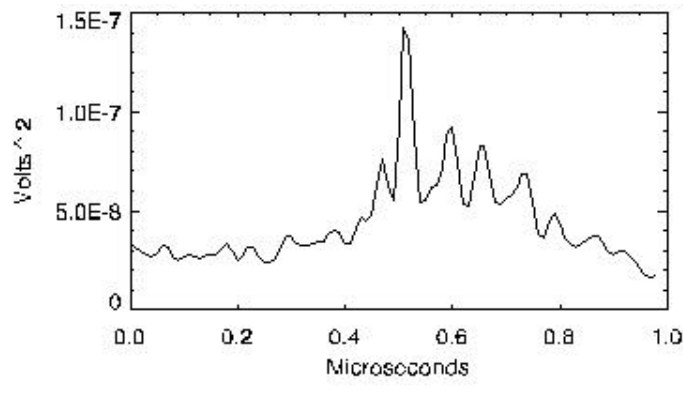

Squared \& Averaged Waveform for Sample \#13A

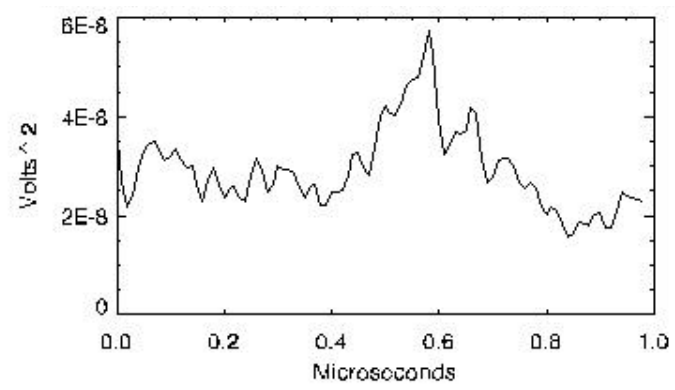

Squared \& Averaged Waveform for Sample \#13B

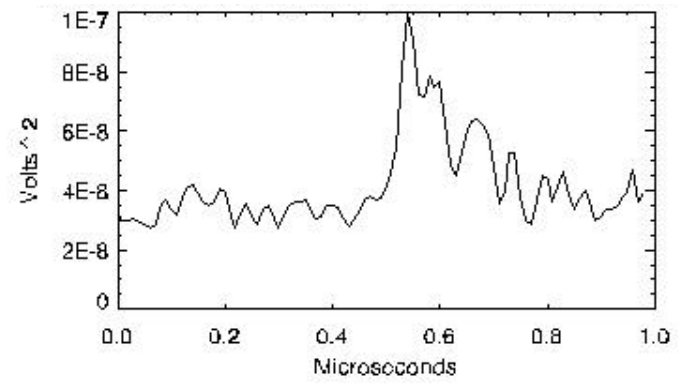

TOF Histogram of Sample \#10A


TOF Histogram of Sample \#13A

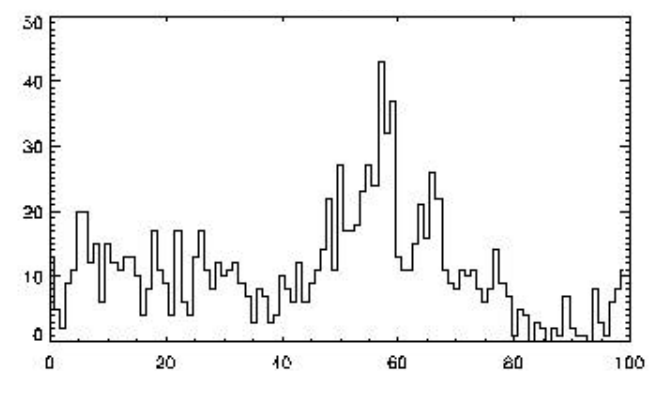

TOF Histogram of Sample \#13B

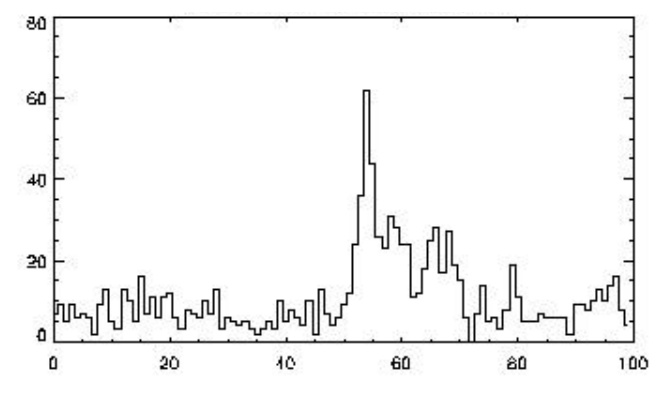


Squared \& Averaged Waveform for Sample \#14A

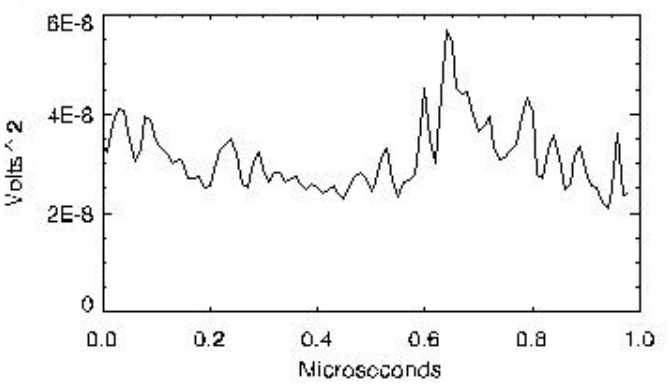

Squared \& Averaged Waveform for Sample \#14B

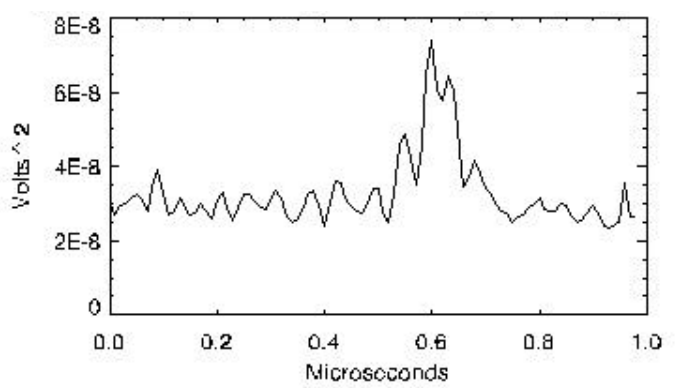

Squared \& Averaged Waveform for Sample \#15A

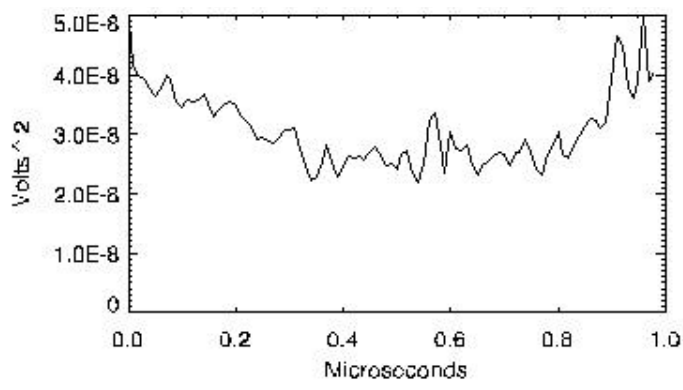

Squared \& Averaged Waveform for Sample \#15B

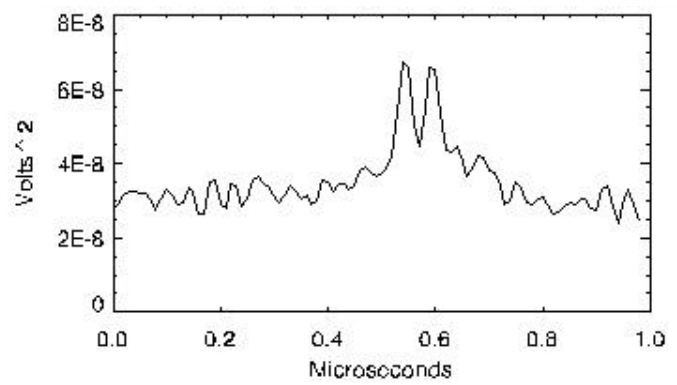

TOF Histogram of Sample \#14A
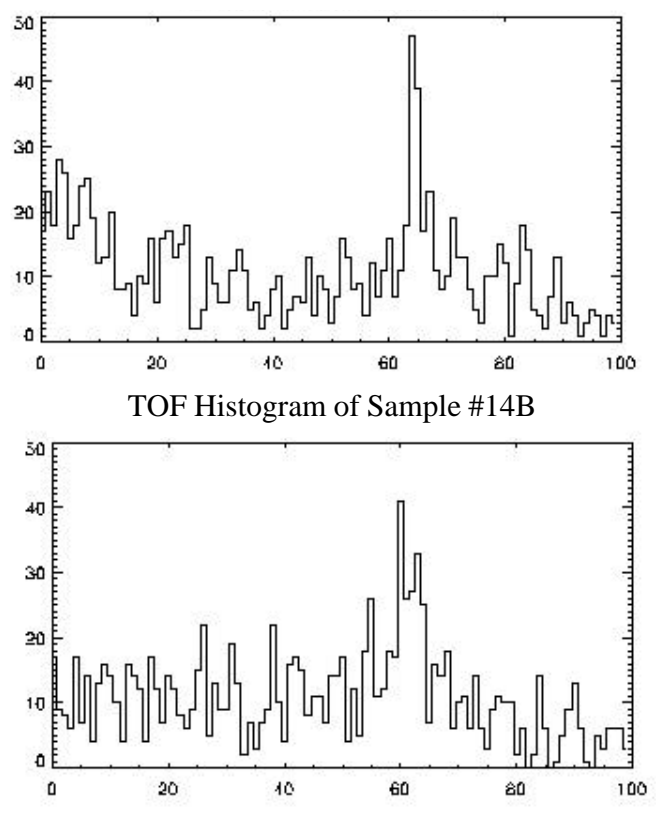

TOF Histogram of Sample \#15A

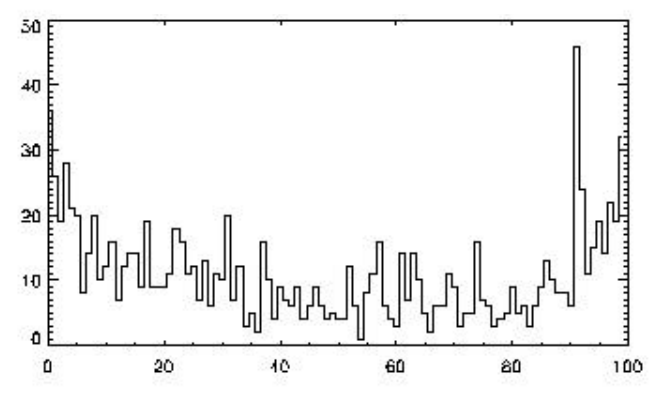

TOF Histogram of Sample \#15B

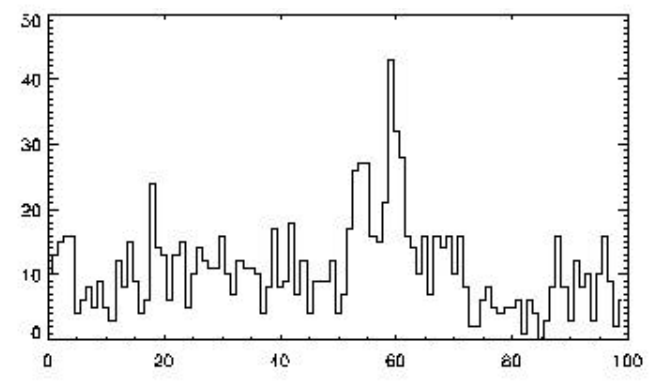


Squared \& Averaged Waveform for Sample \#17A



Squared \& Averaged Waveform for Sample \#17B

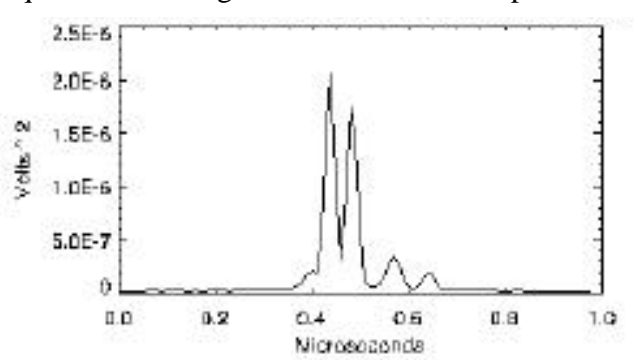

Squared \& Averaged Waveform for Sample \#19A

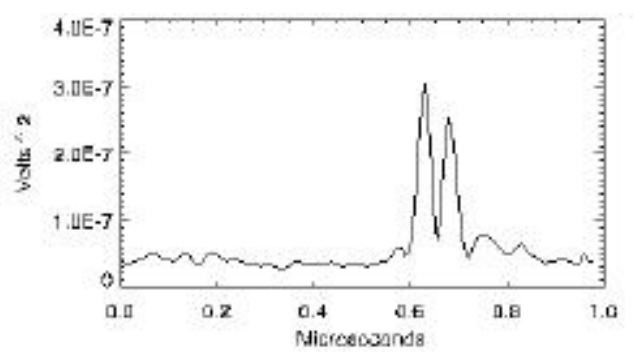

Squared \& Averaged Waveform for Sample \#19B

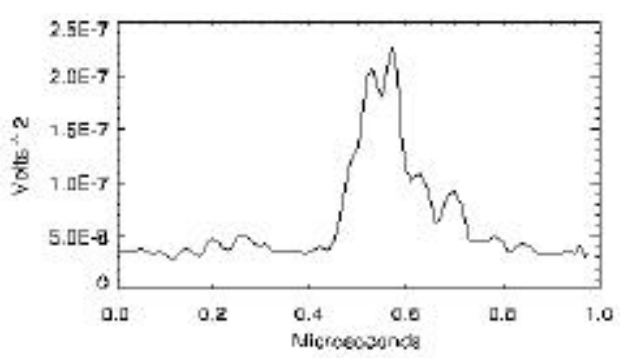

TOF Histogram of Sample \#17A
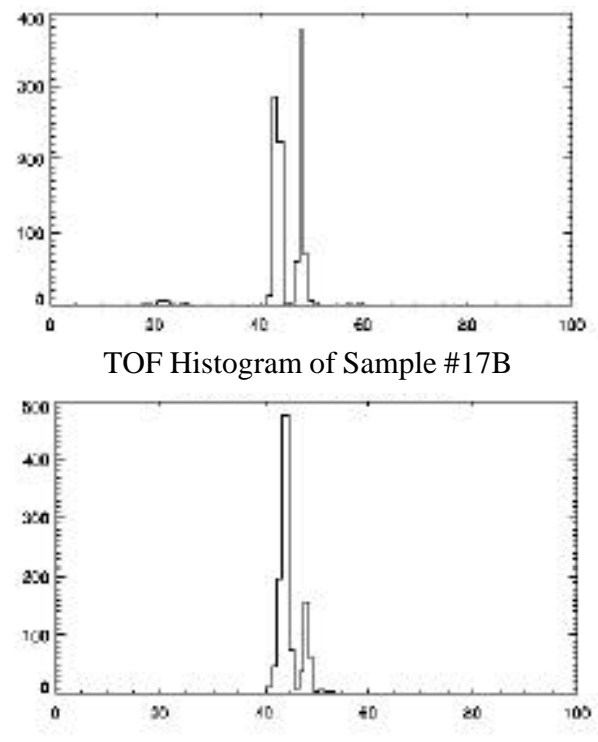

TOF Histogram of Sample \#19A

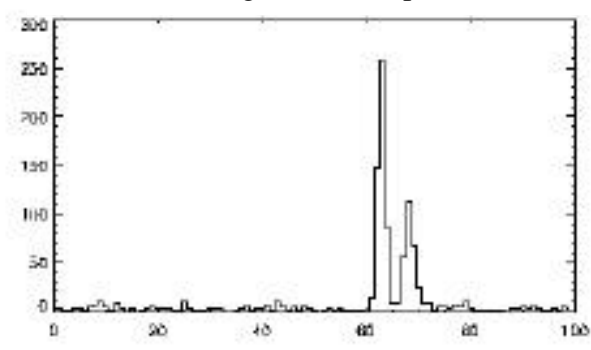

TOF Histogram of Sample \#19B

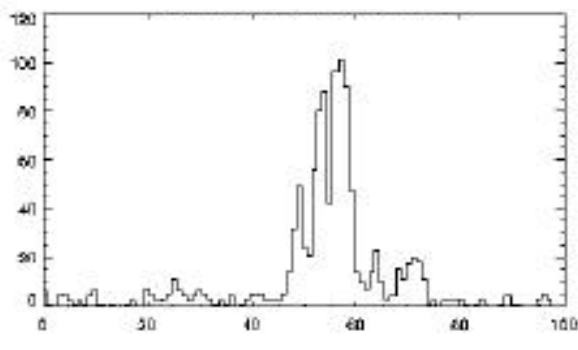




\section{Appendix F}

\section{Metallographic Images of the Bond Interface}




\section{APPENDIX F}

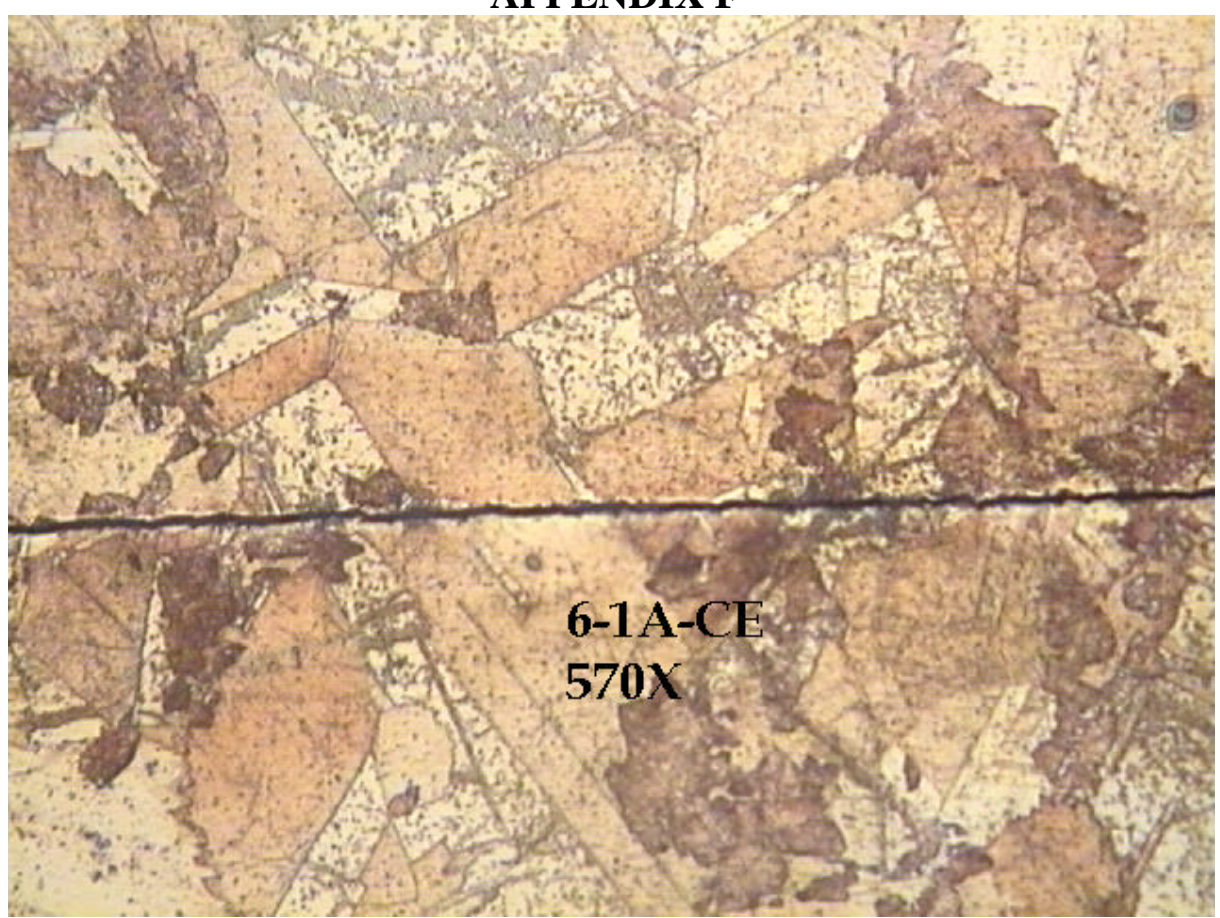

\section{Sample 6-1A-C Cross Section}

Figure F1

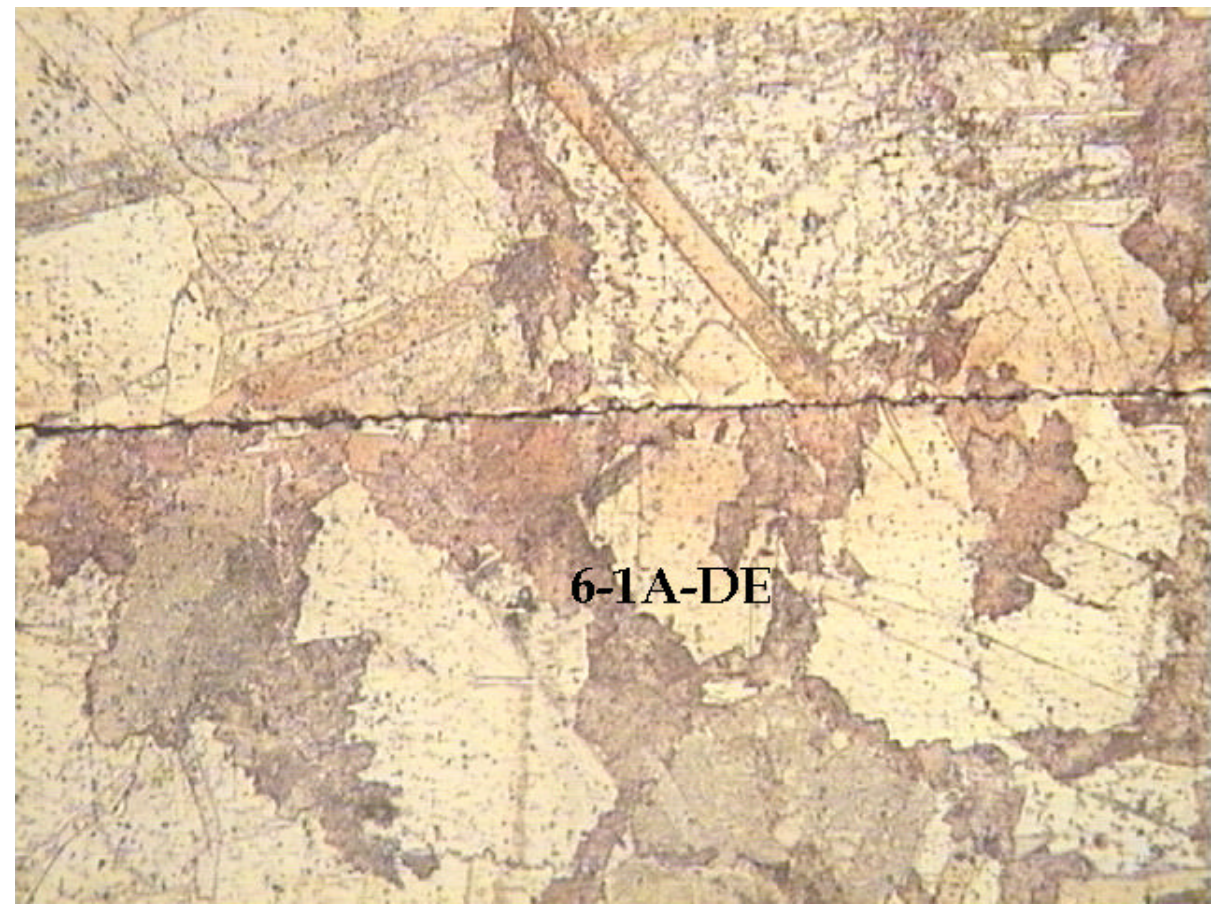

Sample 6-1A-D Cross Section

Figure F2 


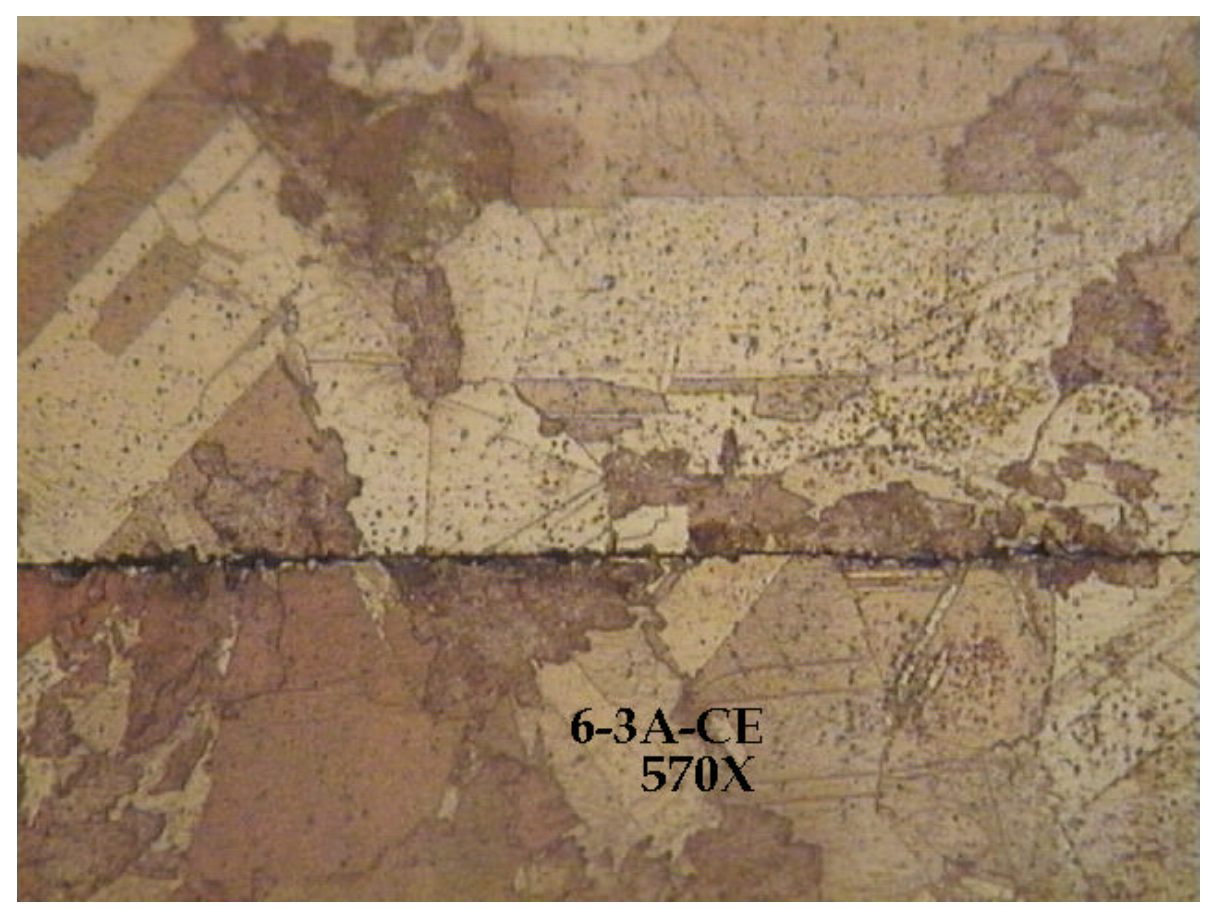

Sample 6-3A-C Cross Section Figure F3

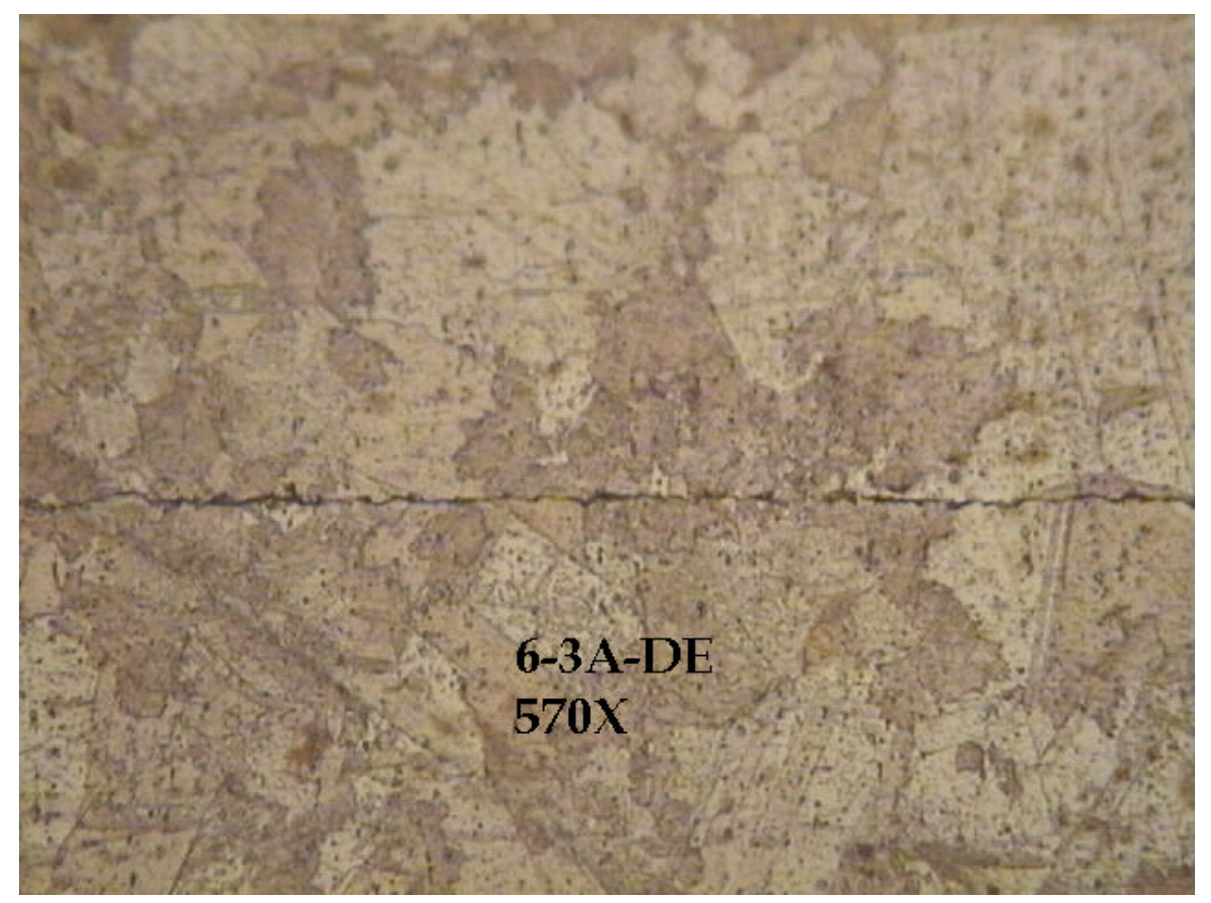

Sample 6-3A-D Cross Section

Figure F4 


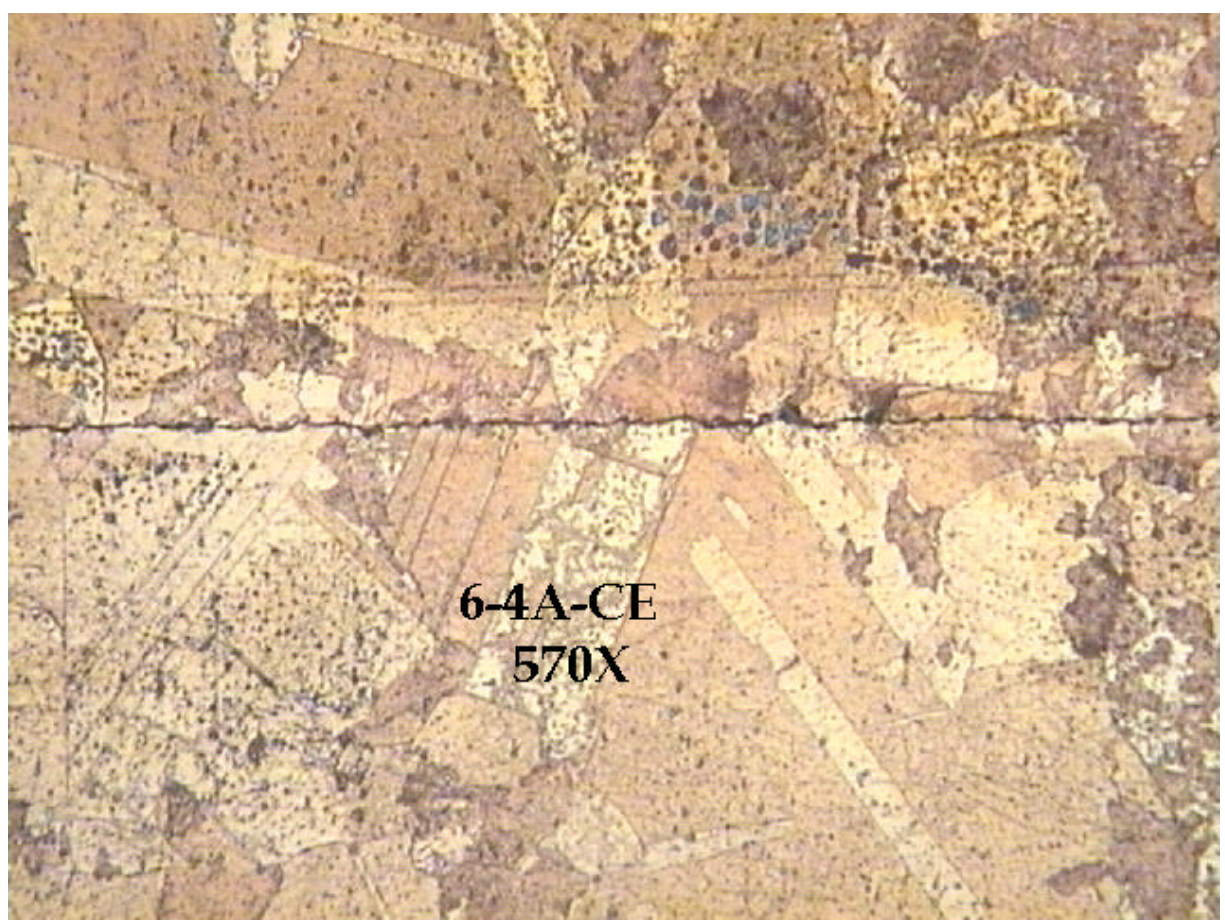

Sample 6-4A-C Cross Section

Figure F5

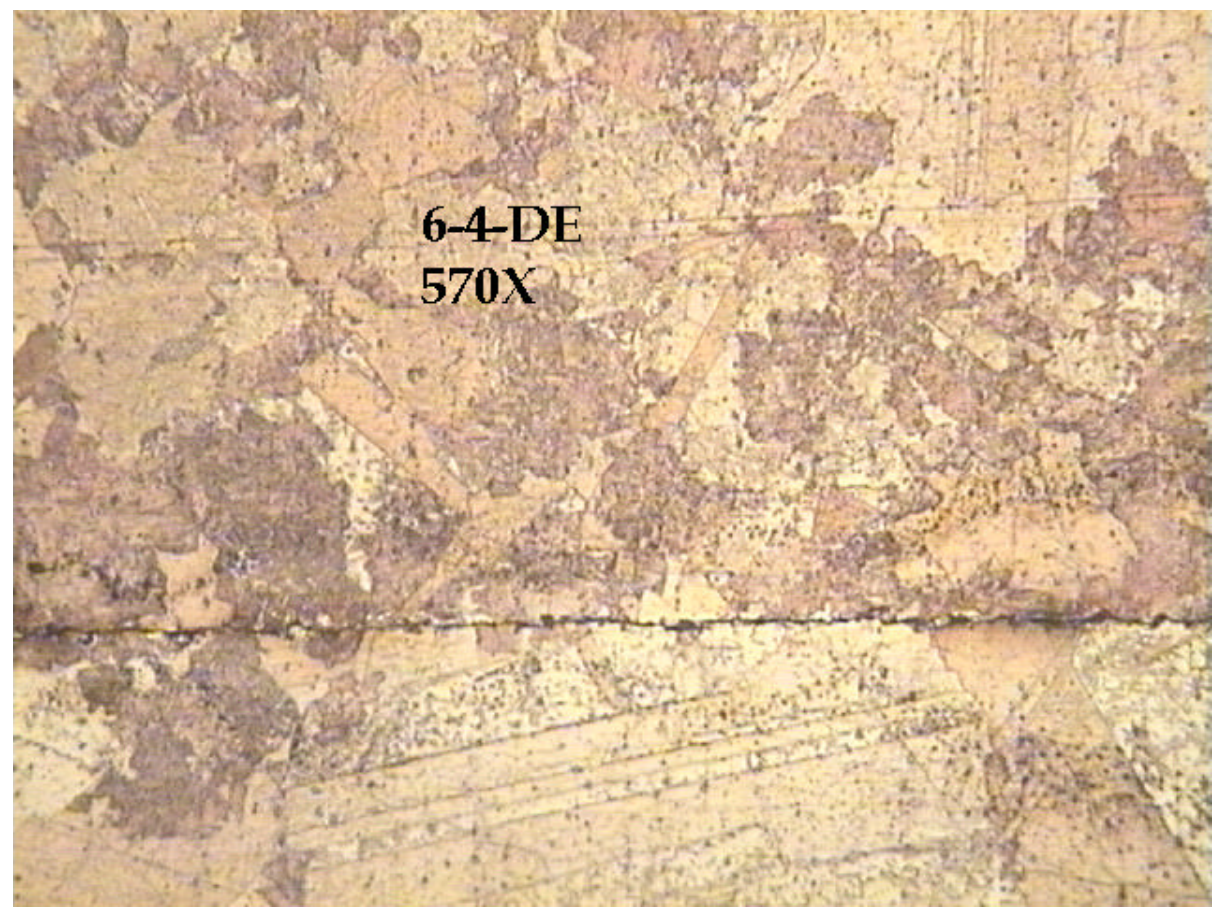

Sample 6-4A-D Cross Section

Figure F6 


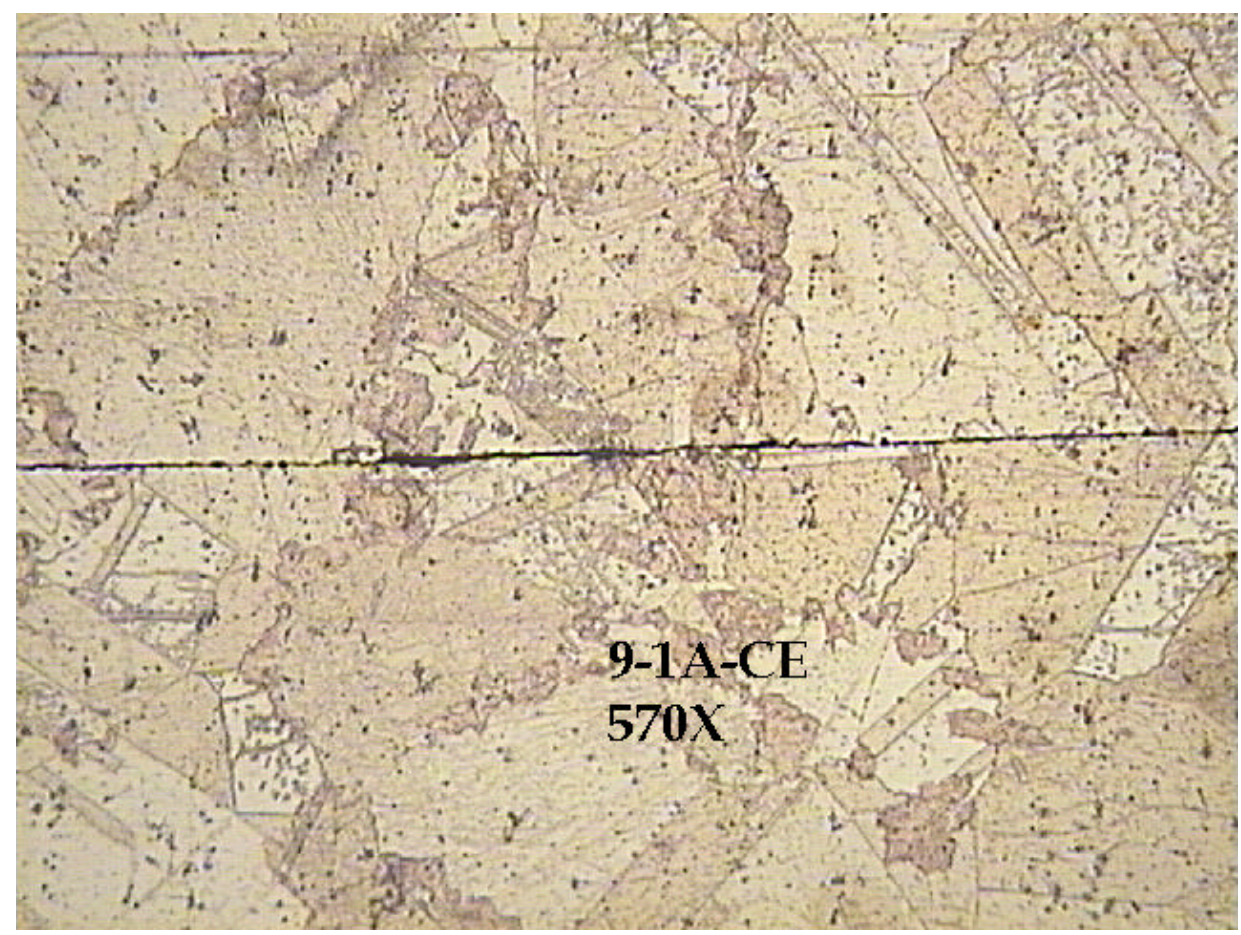

\section{Sample 9-1A-C Cross Section}

Figure F7

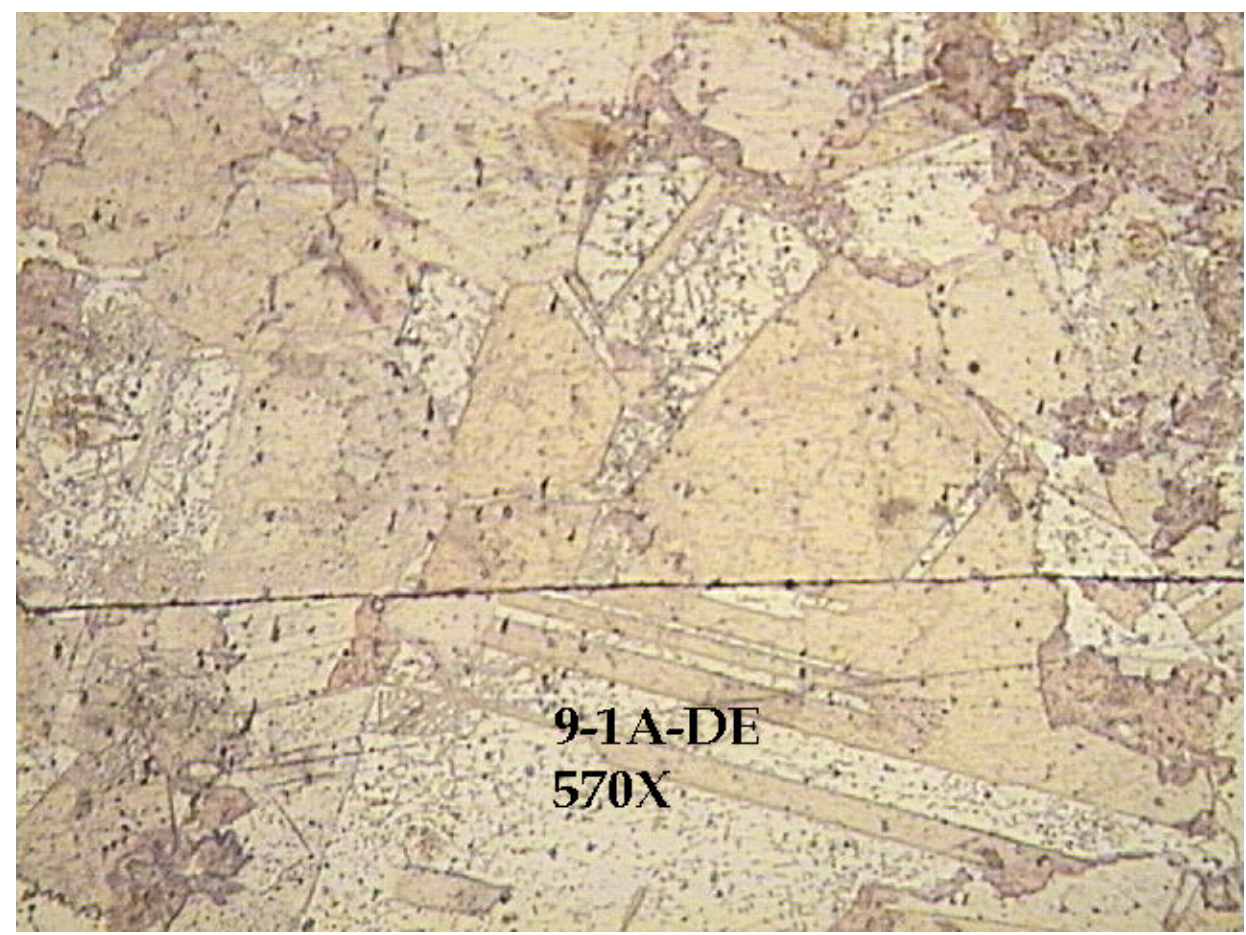

\section{Sample 9-1A-D Cross Section}

Figure F8 


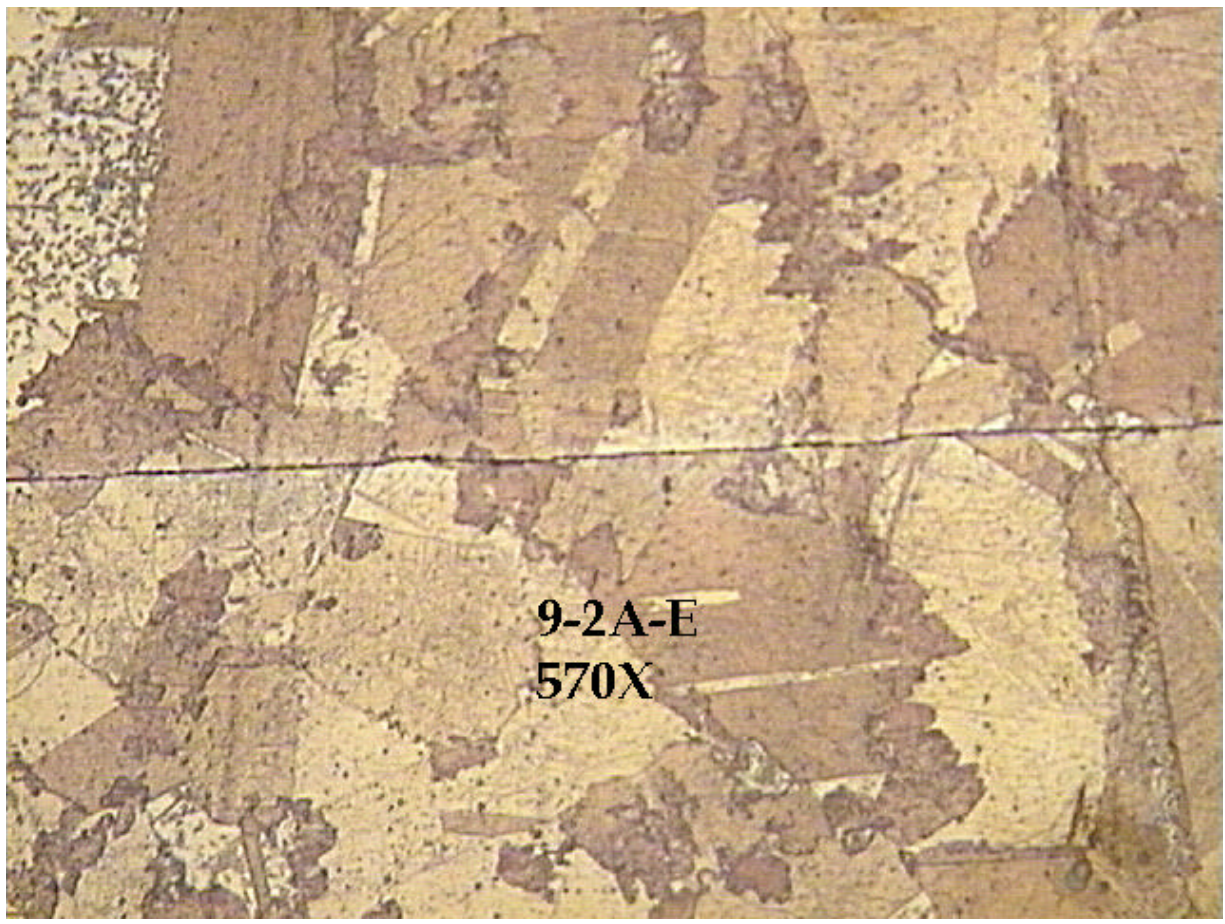

Sample 9-2A-C Cross Section

Figure F9

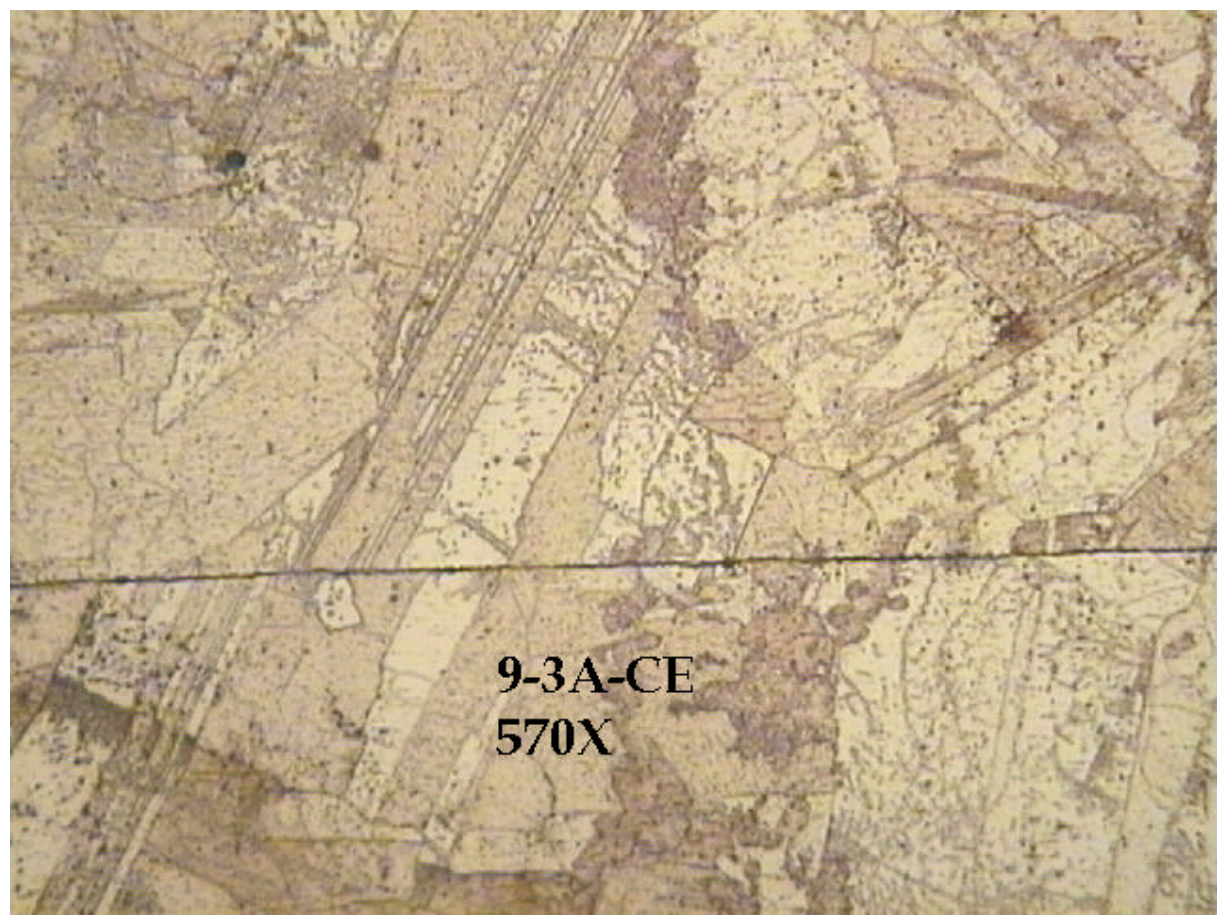

Sample 9-3A-C Cross Section

Figure F10 


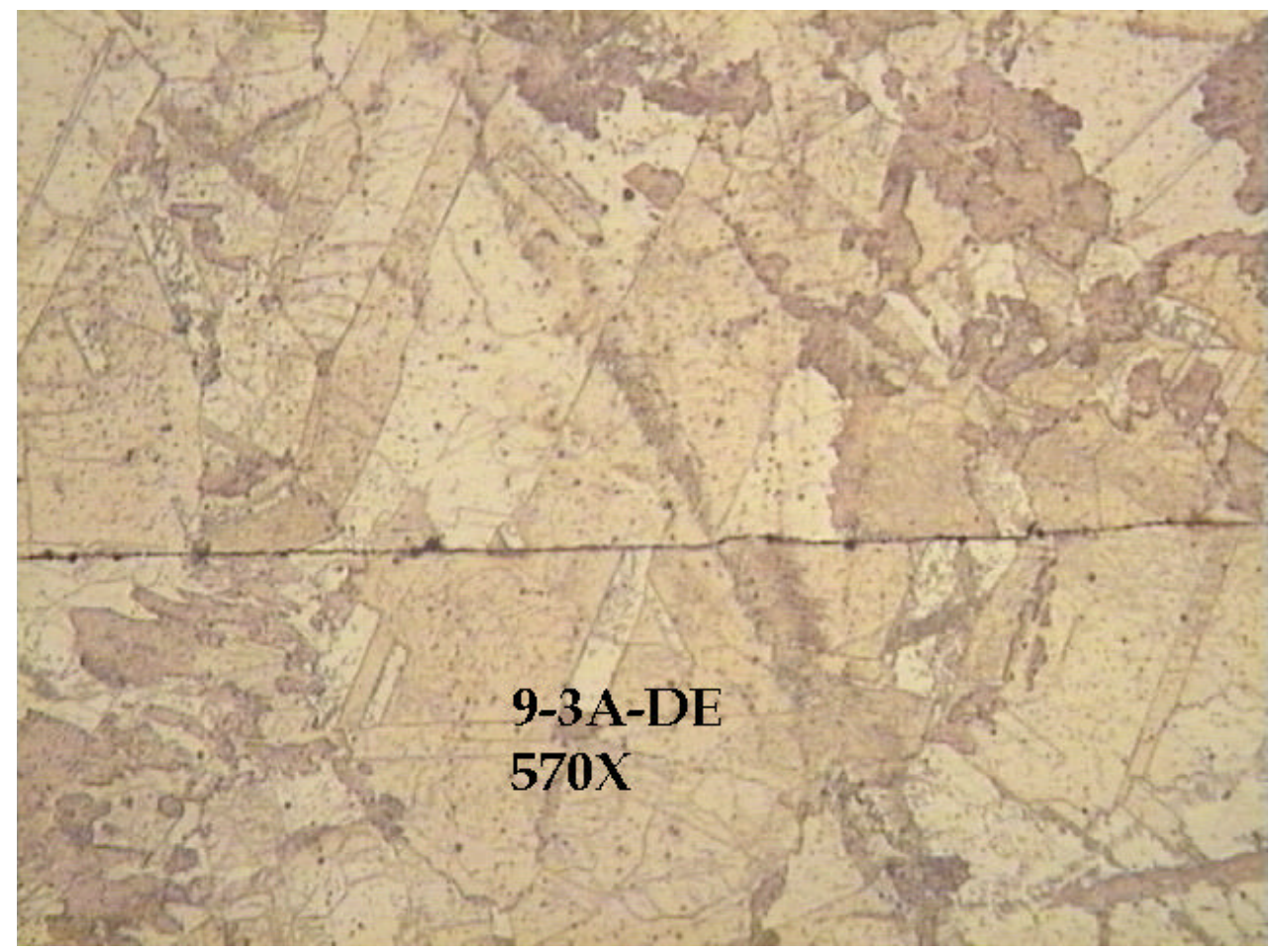

Sample 9-3A-D Cross Section

Figure F11

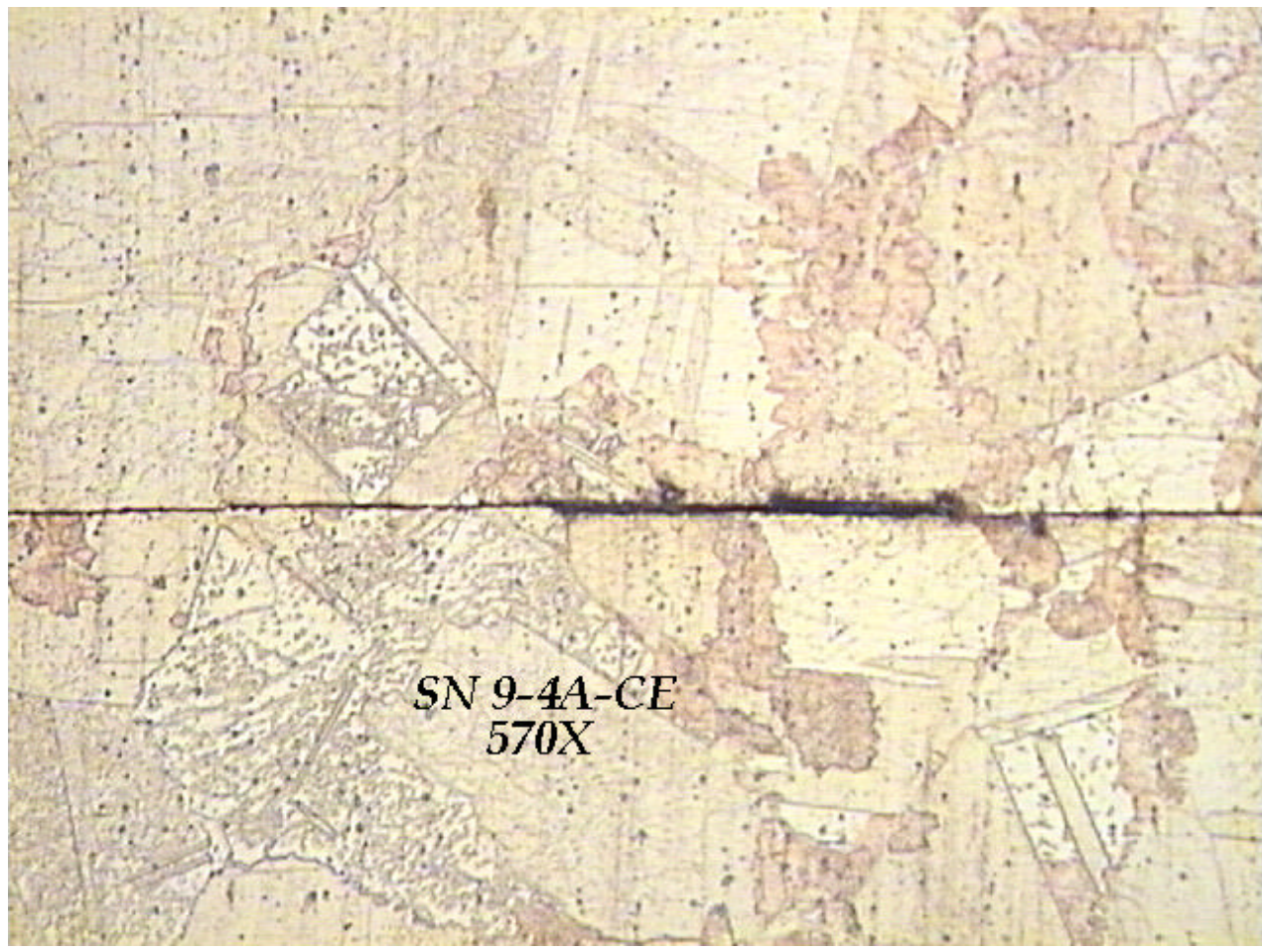

Sample 9-4A-C Cross Section

Figure F12 


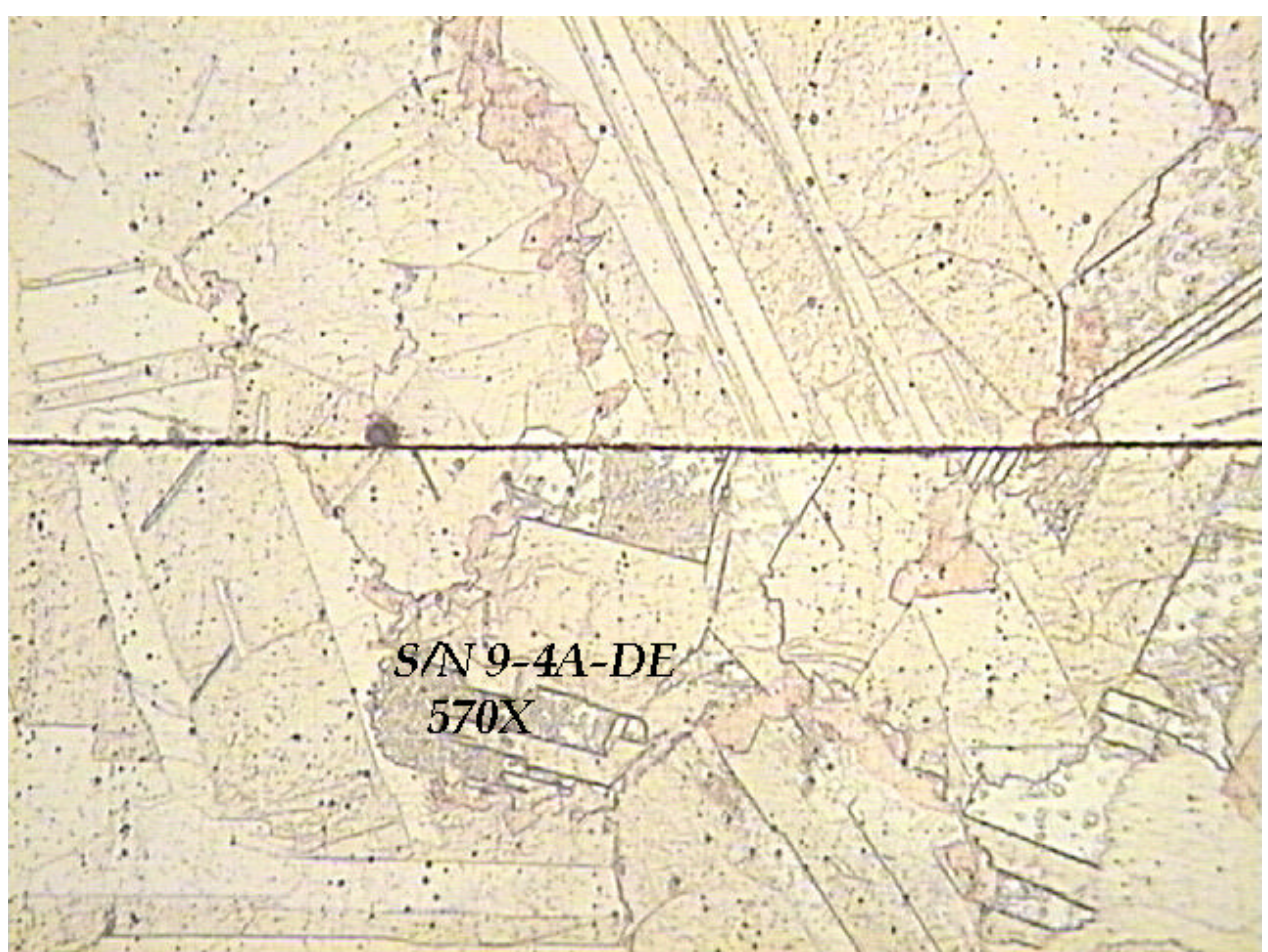

Sample 9-4A-D Cross Section Figure F13



Sample 2 Face A - 1000X

Figure F14 


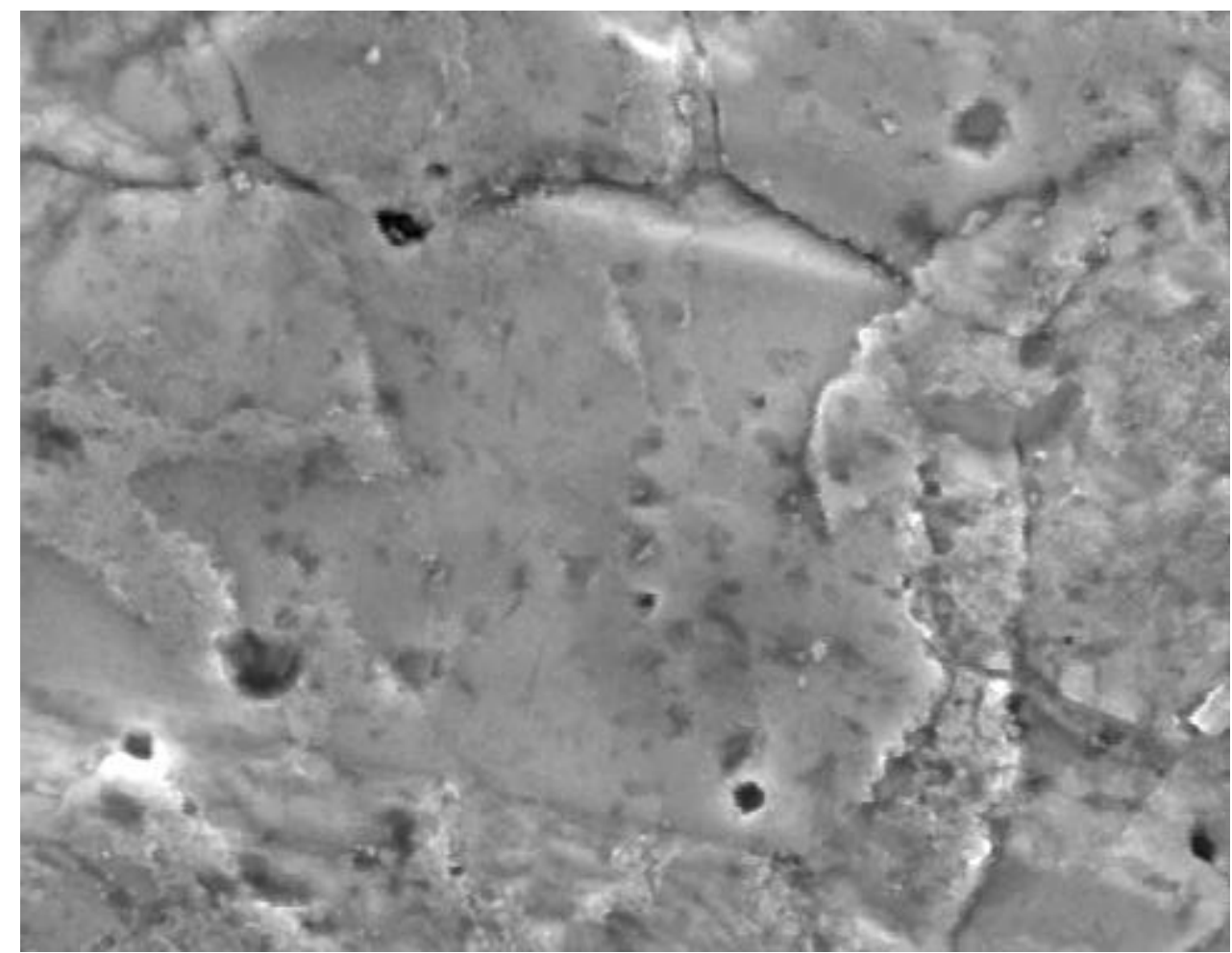

Sample 2 Face A - 5000X

Figure F15

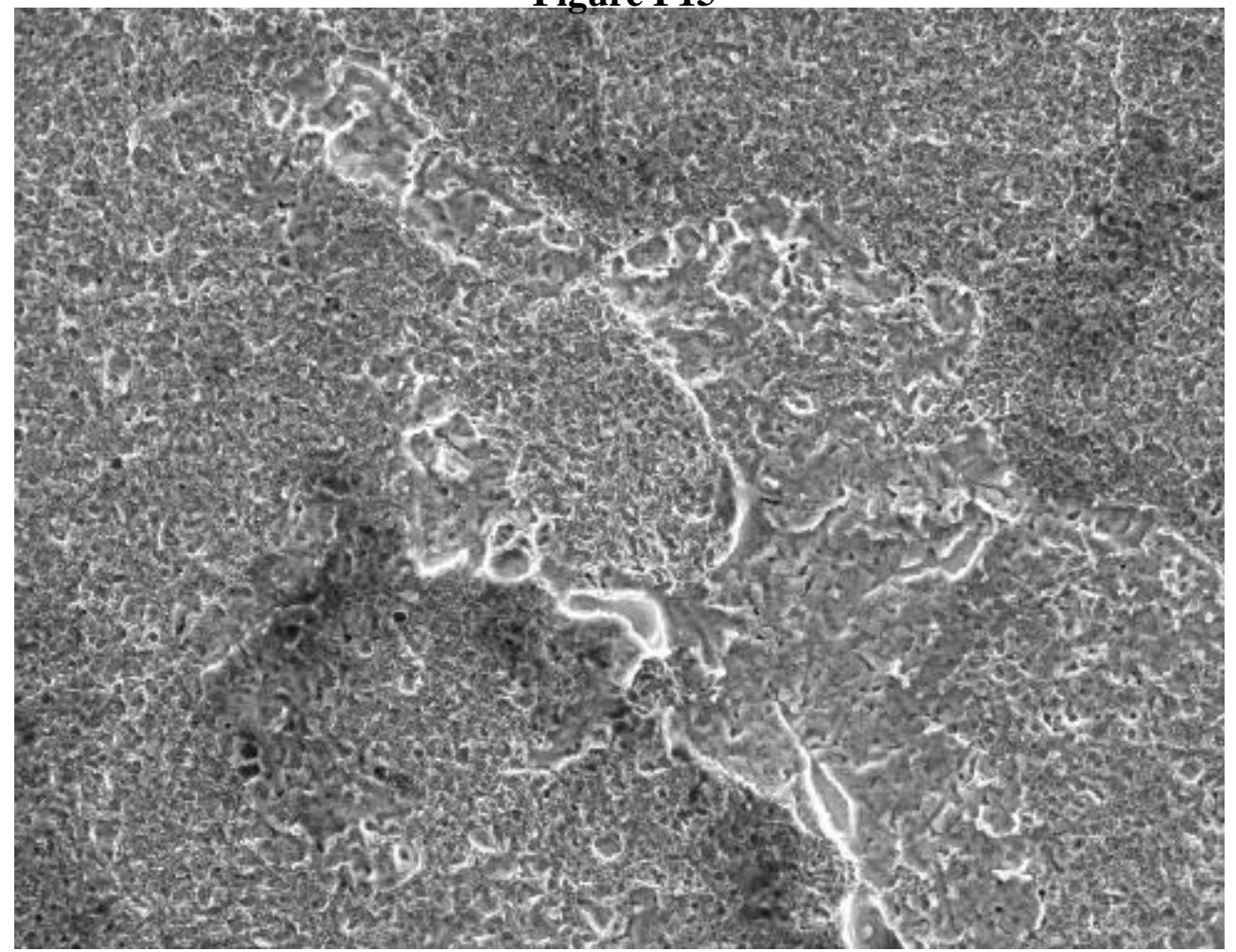

Sample 19 Face A - 1000X

Figure F16 


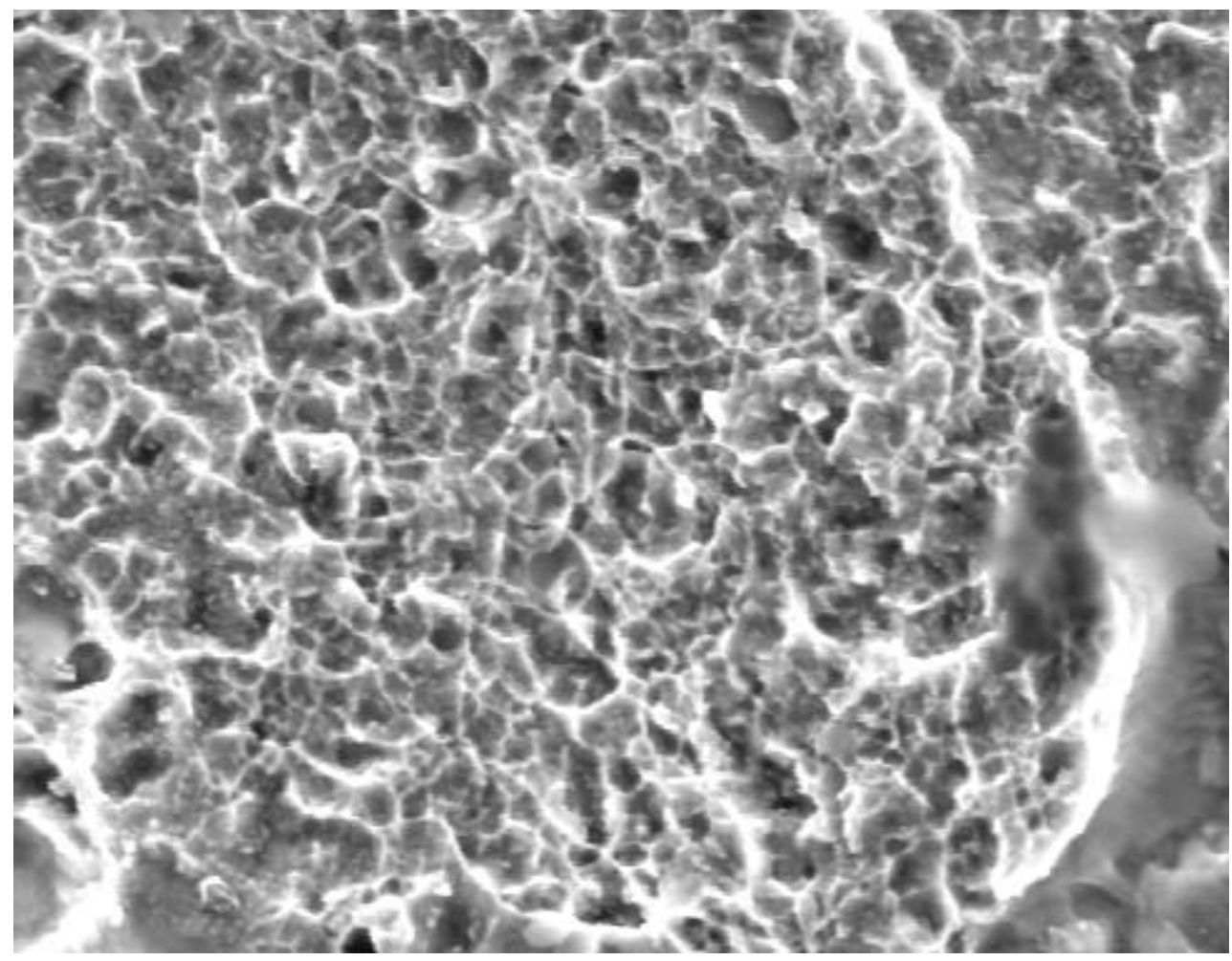

Sample 19 Face A - 5000X

Figure F17

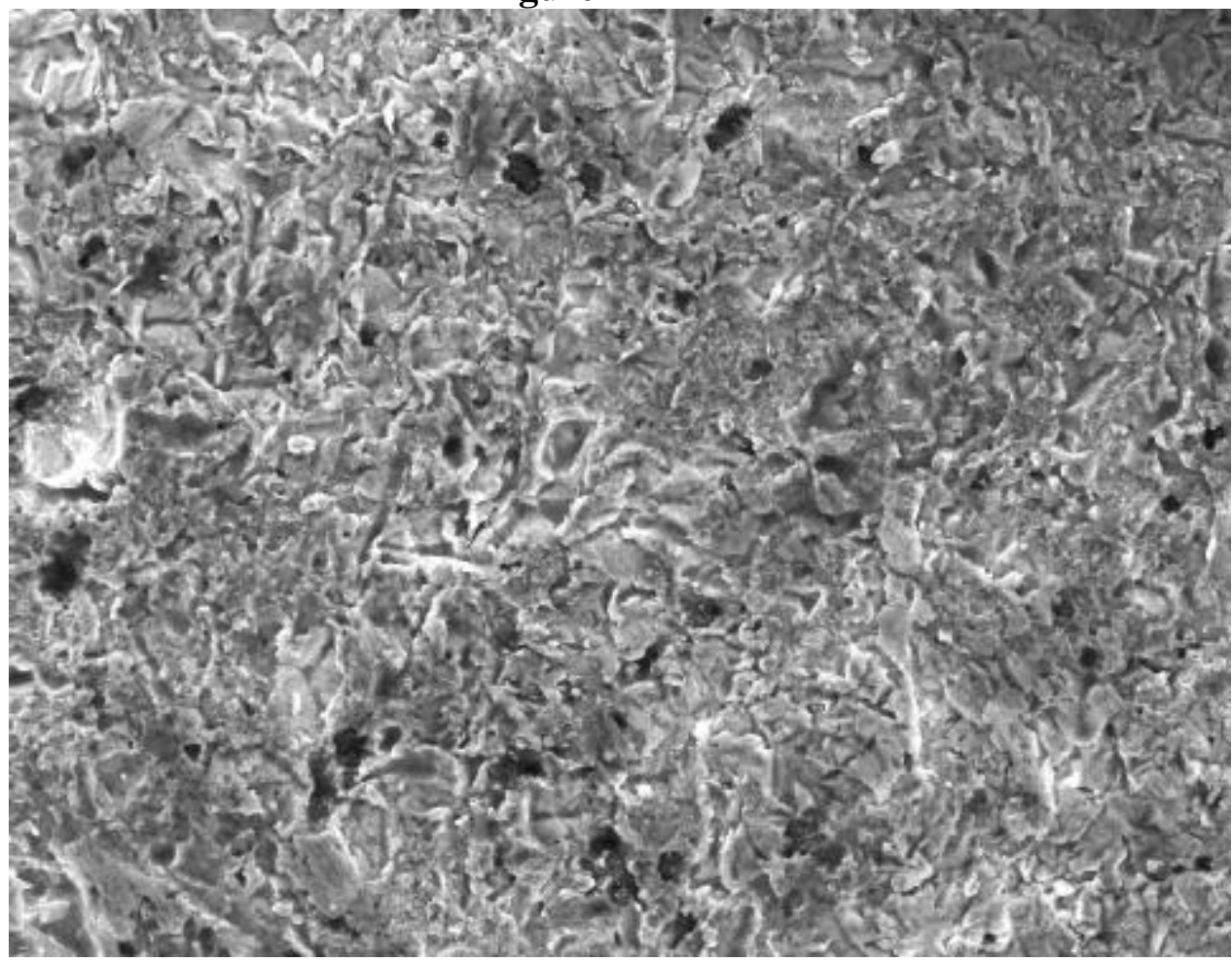

Sample 8 Face A - 1000X

Figure F18 


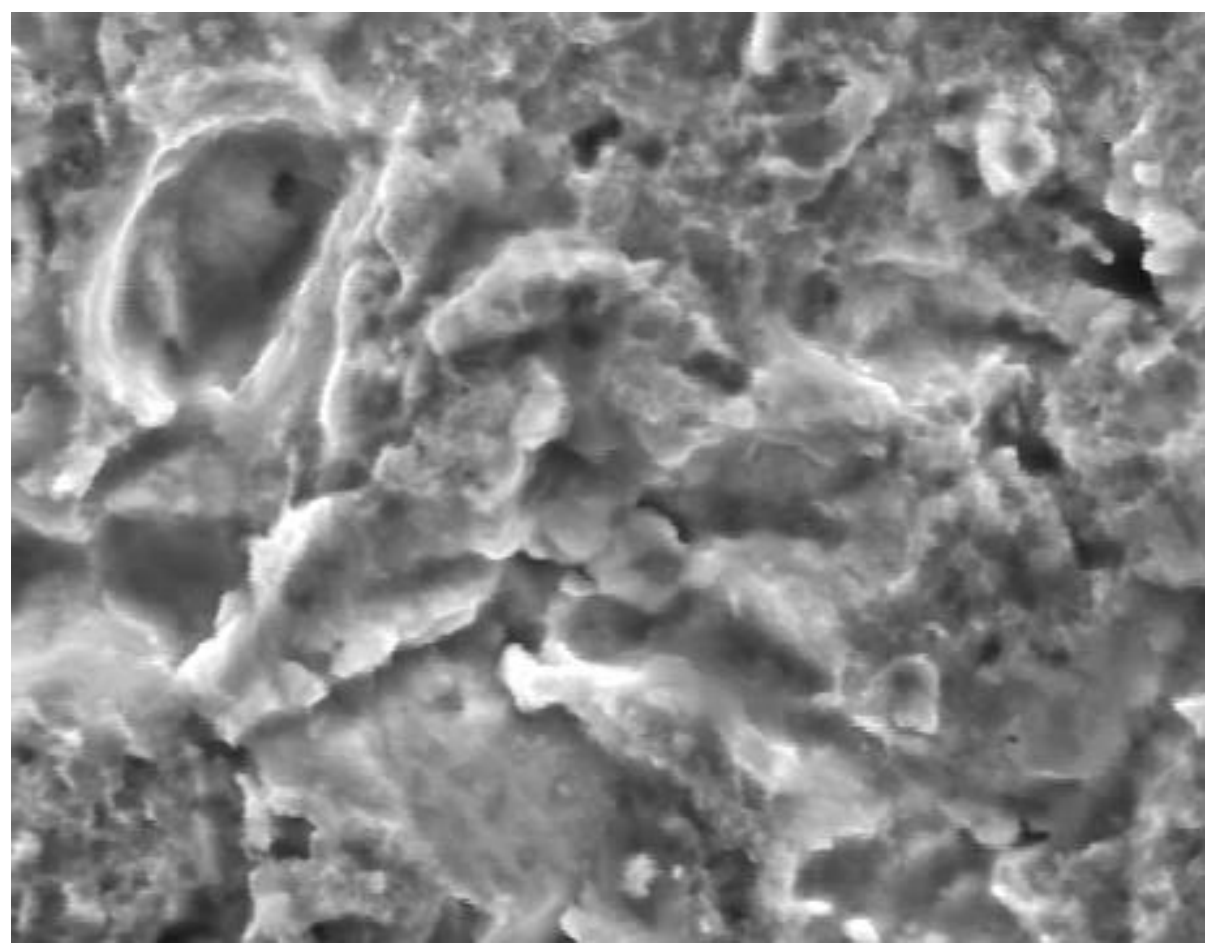

Sample 8 Face A - 5000X

Figure F19 